
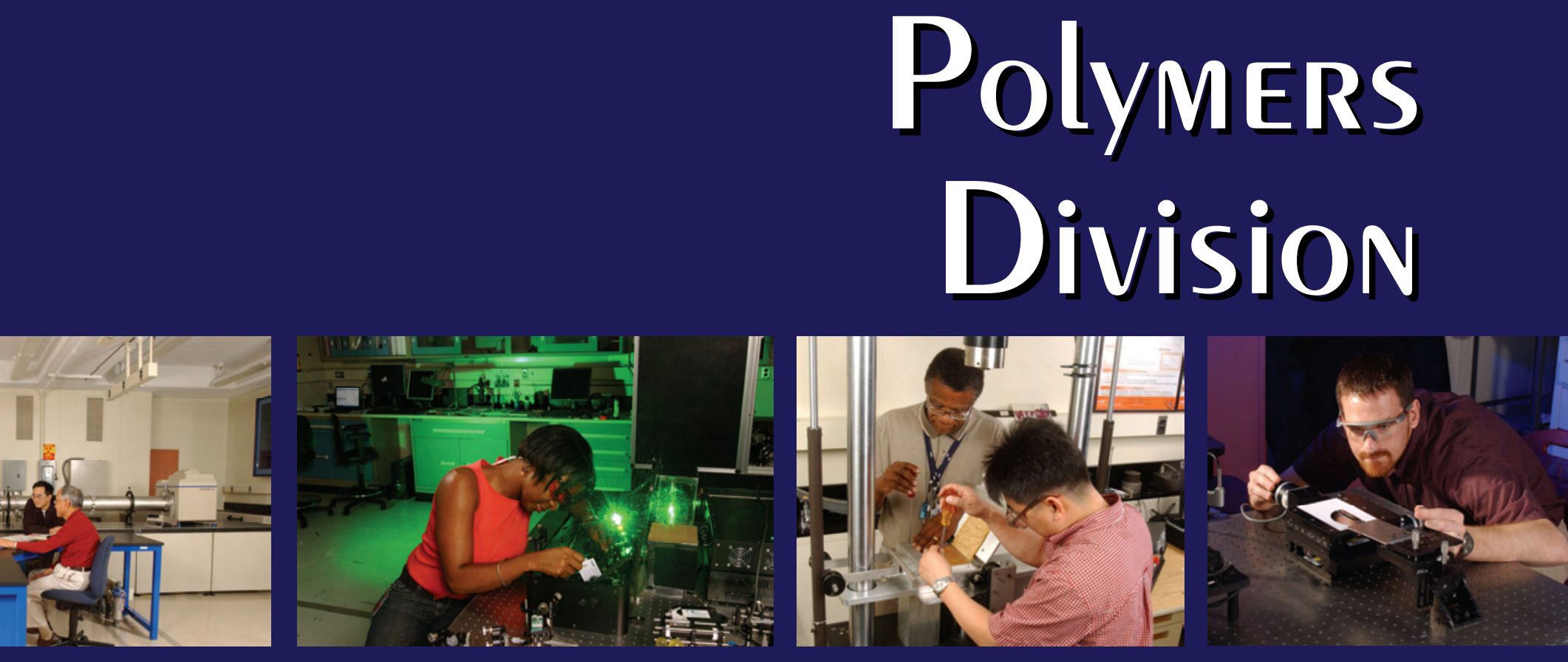

\title{
Materials Science and EngineEring Laboratory
}

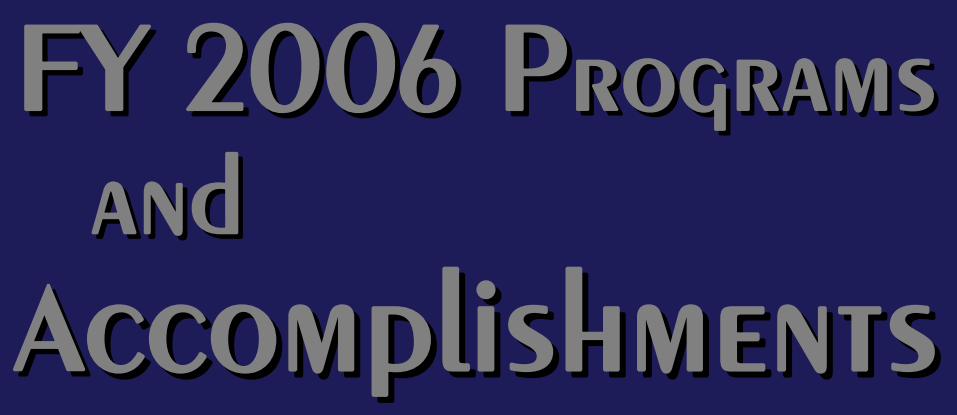

\section{NGT}

National Institute of

Standards and Technology

Technology Administration

U.S. Department of

Commerce

NISTIR 7480

February 2008 
National Institute of

Standards and Technology

James Turner

Acting Director

U.S. Department

of Commerce

Carlos M. Gutierrez

Secretary

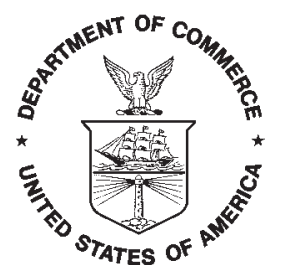

\section{Materials Science and Engineering Laboratory}

\section{FY 2006 \\ Programs and Accomplishments}

\section{Polymers Division}

Eric J. Amis, Chief

Chad R. Snyder, Deputy Chief

NISTIR 7480

February 2008 
Certain commercial entities, equipment, or materials may be identified in this document in order to describe an experimental procedure or concept adequately. Such identification is not intended to imply recommendation or endorsement by the National Institute of Standards and Technology, nor is it intended to imply that the entities, materials, or equipment are necessarily the best available for the purpose. 


\section{Table of Contents}

Executive Summary 1

Technical Highlights

Cell Morphology and Migration Linked to Substrate Rigidity ...........................................2

Chemical Heterogeneity in Chemically Amplified Photoresists:

FTIR Measurements and the Effect on Film Dissolution ................................................. 4

Combinatorial Platform for Measuring the Effect of

Substrate Heterogeneities

Comparative Metrologies for Evaluating Single Wall Carbon

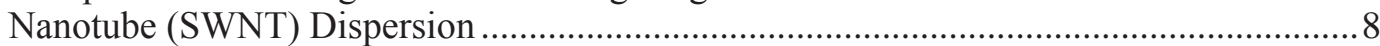

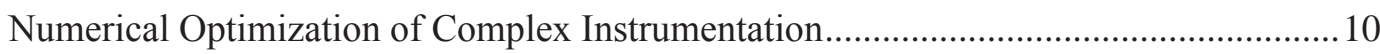

Platform for Characterizing Defects in Magnetic Nanoparticles ......................................12

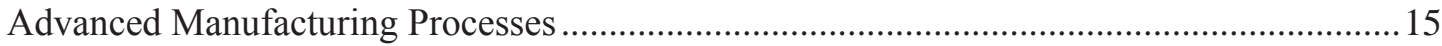

NIST Combinatorial Methods Center:

A Pioneer and Partner in Accelerated Materials Research........................................16

Polymer Formations:

Soft Materials Measurements on a Chip ................................................................... 17

Quantitative Polymer Mass Spectrometry .....................................................................18

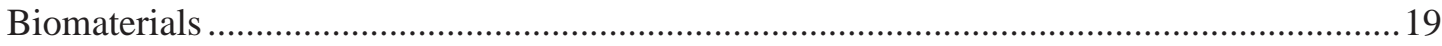

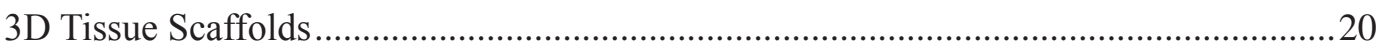

Combinatorial Methods for Tissue Engineering ..............................................................21

Dental Polymers and Composites:

Developing a Test Suite for Total Materials Characterization ....................................22

Metrology for Biomechanical Effects on Cell/Material Interactions ................................23

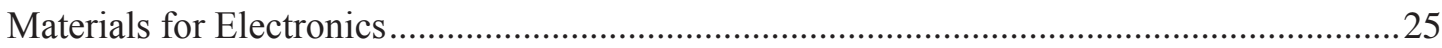

Dimensional Metrology with Small Angle X-Ray Scattering ...........................................26

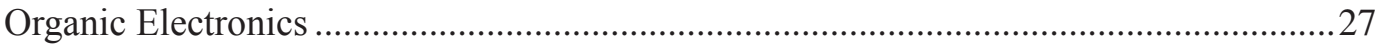

Polymer Photoresists for Next-Generation Nanolithography ........................................28 


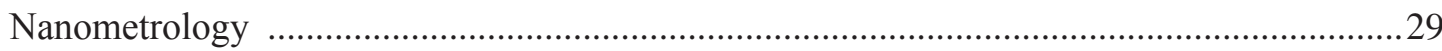

Carbon Nanotube Materials:

Achieving the Gold Standard in Quality and Characterization .......................................30

Combinatorial Methods for Nanomaterials and Nanometrology ....................................31

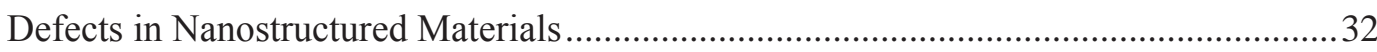

Mechanics of Complex Interfaces:

Libraries for Probing Interfacial Adhesion ..................................................................33

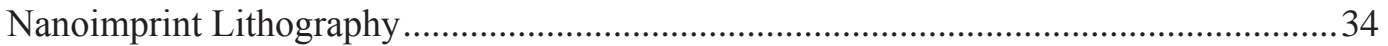

Self-Assembly for Soft Nanomanufacturing ................................................................... 35

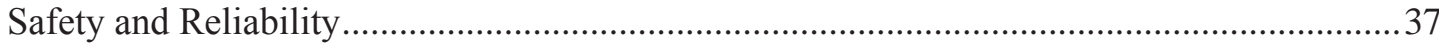

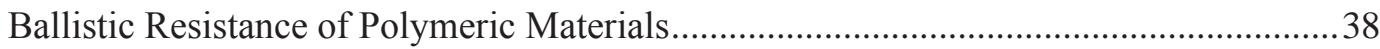

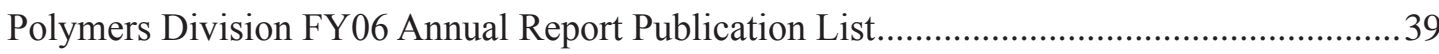

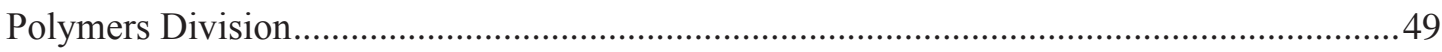

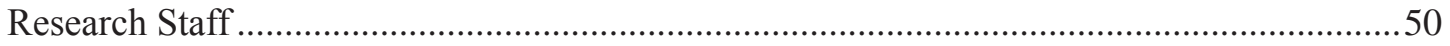

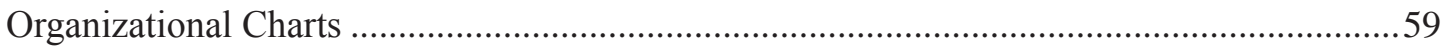




\section{Executive Summary}

I am pleased to yet again report to you the results of a strong year for the Polymers Division. Our staff and research collaborators continue to be acknowledged for their work in important areas, and in my summary this year, I would like to note some of the recognitions received this year.

As an agency of the Department of Commerce, the National Institute of Standards and Technology focuses on work, often in collaboration with industry, to foster innovation, trade, security, and jobs. This year, our efforts have been recognized by an award specifically related to service to industry. The Secretary of Commerce awarded the Department of Commerce Silver Medal for the development of innovative measurement methods for the structure and properties of nanoporous low-k thin films needed in next-generation IC's to Barry J. Bauer, Christopher L. Soles, Da-Wei Liu, Ronald L. Jones, Eric K. Lin, and Wen-li Wu. Silver Medals are awarded for work that demonstrates exceptional performance characterized by superlative contributions that have a direct and lasting impact within the Department of Commerce.

Two Bronze Medals were also awarded within the Division by the NIST Director. Jan Obrzut received his for leadership in development and demonstration of an accurate broadband dielectric measurement for frequencies where earlier methods were inadequate; and Christopher L. Soles received his for innovative applications of incoherent neutron scattering to measure the dynamics of glassy materials. Bronze Medals are awarded for outstanding contributions that increase the efficiency and effectiveness of NIST.

Complementing these awards for service to industry, several scientists and engineers were acknowledged for their outstanding scientific careers. In a White House ceremony on July 26, 2006, Christopher L. Soles was awarded the 2005 Presidential Early Career Award for Scientists and Engineers (PECASE), the nation's highest honor for professionals at the outset of their independent research careers. Chris was recognized for his development of measurement methods and scientific insight into confined nanostructured materials. Annually, two NIST researchers are nominated for PECASE. Chris is the fourth Polymers Division scientist to win this honor with the previous awardees Sharon Glotzer (1998), Eric Lin (2001), and Michael Fasolka (2004).

At the 2006 Annual March meeting of the American Physical Society (APS), Steven D. Hudson was named a Fellow of the Society for excellence in structural studies of supramolecular and polymeric materials and the quantative description of droplet and particle dispersion under quiescent and flow conditions. Steve joins seven other current Polymers Division scientists and dozens of Division alumni as APS fellows.

Annually, our report provides a sample of the outstanding research from the Polymers Division. As usual, only a portion of our work is included in this report, so please visit www.nist.gov/polymers for more details. On our site, you can also download copies of any of our publications. As always, I welcome your comments.

This also represents my last report to you as Chief of the Polymers Division. With the start of the 2007 fiscal year, I moved to our laboratory office as Deputy Director of Materials Science and Engineering. I have thoroughly enjoyed my 11 years working with the outstanding team of talented and dedicated scientists in this premier organization. Our research continues to push the scientific boundaries and fulfill the NIST mission. It is with heartfelt appreciation that I thank the staff of the Division and our many alumni for their support as we have grown and learned together. I am especially grateful to the members of my management team, Chad Snyder, Eric Lin, Alamgir Karim, Kalman Migler, Mike Fasolka, Marc Cicerone, and Dawn Bradley, for their dedication, willingness to challenge the status quo, and respect they show to our goals. The same can be said for the many great individuals who make up the Division staff, our collaborators, and the NIST management. I know this team will continue to grow as they give their support to Eric Lin as the new Division Chief. Together we will continue the tradition of excellence of the NIST Polymers Division.

Eric J. Amis

Chief, Polymers Division

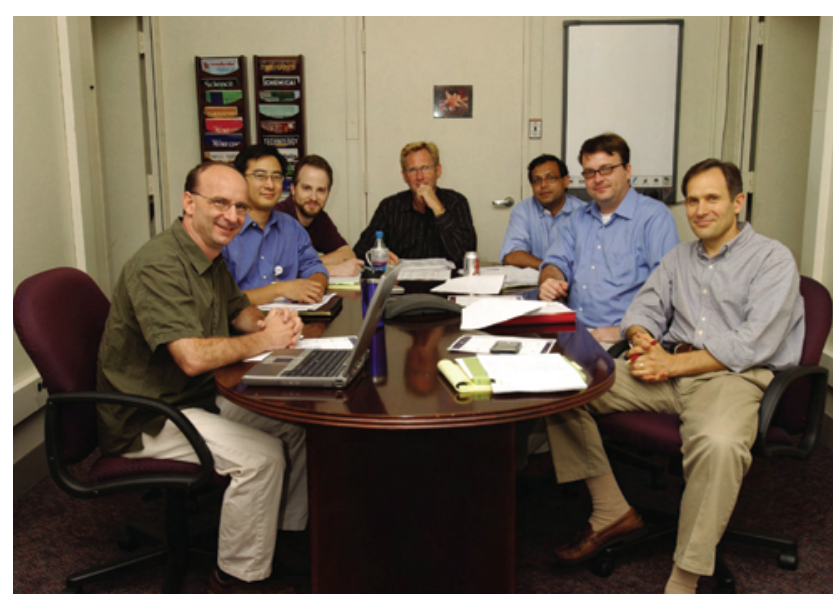

Polymers Division Management Team. Clockwise from Left: Kalman Migler, Eric Lin, Chad Snyder, Eric Amis, Alamgir Karim, Mike Fasolka, and Marc Cicerone. 


\section{Cell Morphology and Migration Linked to Substrate Rigidity}

Appropriate cell migration is critical to many areas of biology, and to guiding outcomes in Tissue Engineering. Also, cell morphological changes affect the formation of cell adhesion and phenotypic expression. Cells have been shown to change morphology and migrate in response to a wide number of external stimuli, including chemical, mechanical, and electrical. We have developed a model that links cell morphology and migration to substrate mechanical properties.

\section{Martin Y.M. Chiang}

Cells cultured, in vivo, on substrates show activity that is influenced by substrate mechanics in the following ways:

(1) Cells respond to variation in substrate rigidity. Certain types of cells on stiffer substrates have less rounded morphologies and are more likely to extend into branched morphologies than the same cells on softer substrates (Fig. 1a). In contrast, some experimental studies show that other cell types extend into more branches on softer substrates and exhibit no preference to branching on stiffer ones (Fig. 1b).

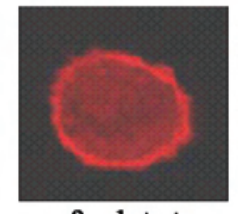

soft substrate

(a)

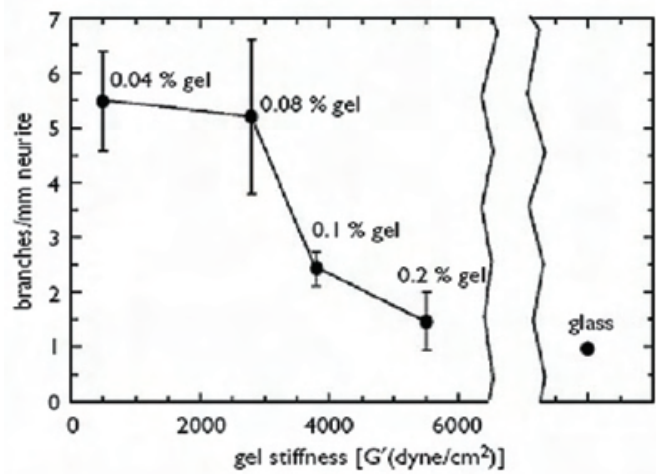

(b)

Figure 1: Morphological change (stability).

(2) When cells, cultured on a substrate, are subjected to a uniaxial cyclical substrate stretch, the cells tend to elongate perpendicular to the direction of stretch

(Fig. 2).

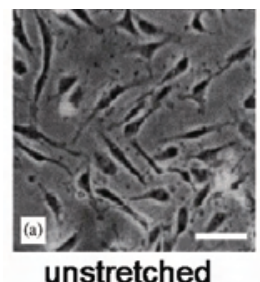

unstretched

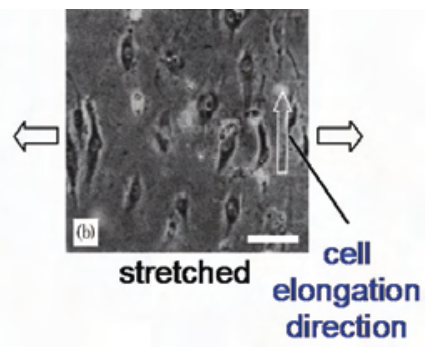

Figure 2: Orientation stability.

(3) Cells tend to migrate towards stiffer regions on a substrate with a stiffness gradient, which is known as durotaxis, although reverse durotaxis (migration towards less stiff regions) has also been observed.

Guided by the aforementioned experimental observations, we have developed a mathematical model of cellsubstrate adhesion that connects cell morphology and migration with the mechanical properties of the extracellular environment. The model is based on the hypothesis that morphology of a cell adhering to a substrate is driven by the competition between strain energies (in the cell and substrate) and interfacial energy (work of adhesion at the cell periphery). The stability of a given cell morphology is given by the total energy of the cell/ substrate system based on strain and interfacial energies.

The total free energy $\left(E_{\text {tot }}\right)$ is expressed as the sum of the strain energy in the cell and substrate $\left(E_{1}\right)$ and interfacial energy at the cell periphery $\left(E_{2}\right)$. The ratio of these energies is given the designation $\beta$ :

$$
\beta=E_{1} / E_{2}
$$

In Fig. 3, the graph on the right hand side of the figure displays $E_{\text {tot }}$ as a function of perturbation mode for several values of $\beta$. Here, $\beta$ is varied by changing substrate rigidity. The image insets correspond to the most
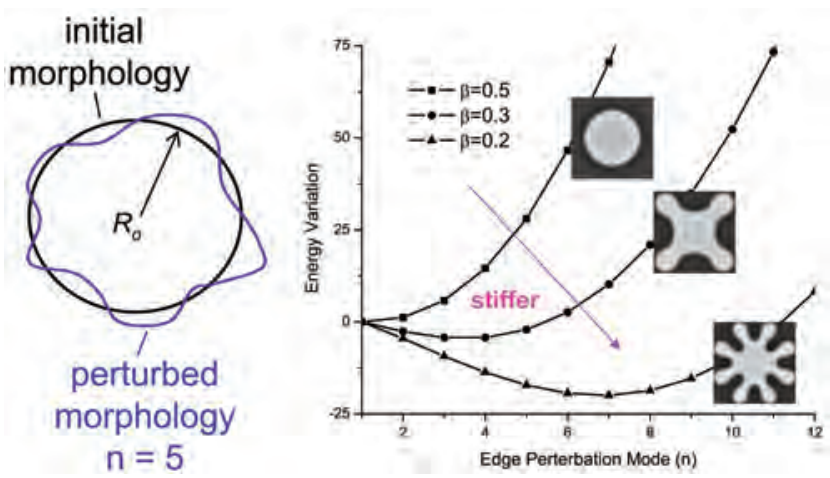

Figure 3: Left-An initially circular cell (solid line) undergoing a morphological perturbation of harmonic form (dotted line). Right-The energy variation as a function of perturbation mode (or number of lobes) $n$ for different values of $\beta$ due to changes of the substrate rigidity. 
stable configuration of the cell predicted using phasefield simulation that includes geometric nonlinearity.

In agreement with experimental observations reported in the literature, our model has demonstrated the hypotheses that the morphological stability of an adherent cell to substrate is governed by the minimal total free energy in the cell/substrate system. The morphological changes of a cell can be rationalized as the consequence of competition between interfacial and elastic energies in the total free energy of cell/substrate system. Thus, the cell changes into its energetically favorable shape by the assembling/disassembling of focal adhesions distributed around the cell periphery. The interfacial energy between the cell and substrate stabilizes the cell morphology while the strain energy from intracellular and extracellular forces destabilizes it. The competition results in a "resonant" window that refers to the range of the stiffness ratio $\left(\mu^{\mathrm{s}} / \mu^{\mathrm{c}}\right.$, substrate to cell) over which the cell is in interaction with the substrate (Fig. 4). With this interaction, the cell and substrate can be mutually compliant. Consequently, it becomes energetically favorable for the cell to undergo significant morphological changes. More importantly, the results from our analysis indicates that the change of trend in morphological stability (the inflection point, Fig. 5) corresponds to $\mu^{s}=\mu^{c}$. Therefore, controlled experiments can be planed to estimate the cell stiffness by observing this change (as shown in Fig. 4.)

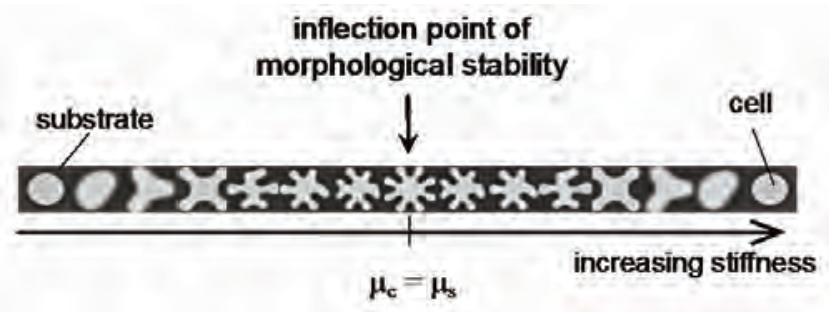

Figure 4: Schematic representing how cell morphology changes in response to substrate rigidity.

Within the framework of our phase-field model, cell migration and orientation are due to the variation of substrate stiffness and durotaxis is viewed as a progressive minimization of the total free energy in a cell/substrate system. Our analysis indicates that the cell migrates not only from softer towards to stiffer regions of the substrate, but that the reverse is possible under certain conditions. The occurrence of durotaxis or reverse durotaxis phenomena depends on the relative rigidity between the cell and substrate.

Our study provides insights into the dependence of cell morphology and migration on substrate mechanics; it can be used to rationalize discrepancies on the trend of morphological changes reported in the literature as well as cell locomotion due to the stiffness variation of a substrate. Finally, the study provides a guide in measurement science for cell stiffness and can be extended to explore cell morphology and migration in a more complex native environment subjected to other external stimuli.

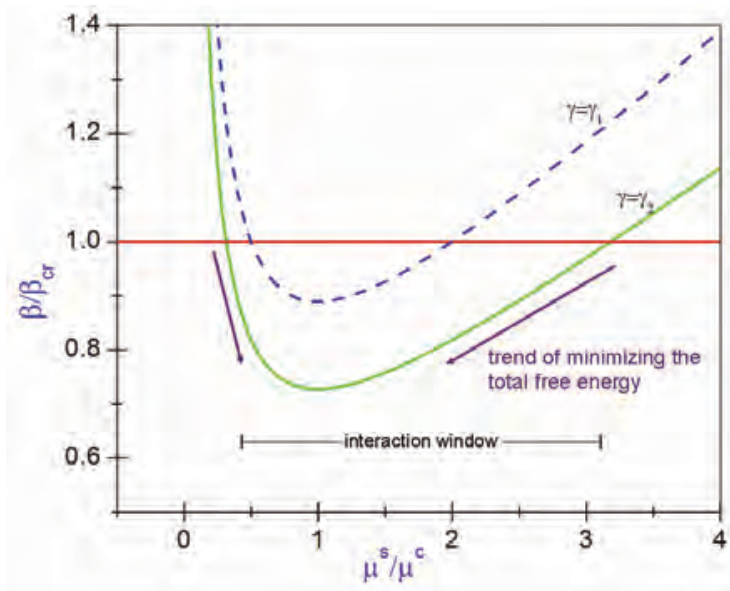

Figure 5: The variation of normalized $\beta$ as a function of rigidity ratio, $\mu^{s} / \mu^{c}$, for various interfacial energies, $\gamma_{1}$ and $\gamma_{2}\left(\gamma_{1}>\gamma_{2}\right)$, corresponding to different cell/substrate systems. $\beta$, the ratio of the interfacial energy density to strain energy density of the cell/ substrate system. The decrease of $\beta$ gives rise to morphological instabilities.
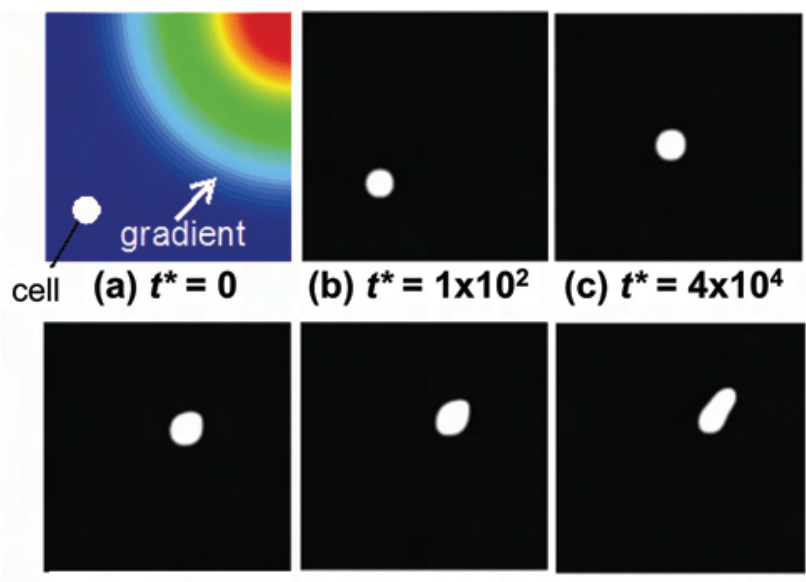

(d) $t^{*}=7 \times 10^{4}$

(e) $t^{*}=9 \times 10^{4}$

(f) $t^{*}=1 \times 10^{5}$

Figure 6: A typical evolution path of the cell from the initial stage to final stage of the simulation. A circular cell initially located on the softer region of substrate with a stiffness gradient (a). The cell migrates from the substrate surface with lower stiffness to higher stiffness ( $b-f)$. When the cell migrates to a region on the substrate surface with a critical stiffness, the cell morphology becomes unstable and changes from the original shape to a "potato"-like shape ( $f$ ). This phenomenon is attributed to a consequence of the competition between energies for the morphological change and the migration.

\section{For More Information on This Topic}

M.Y.M. Chiang, J.P. Dunkers, and M.T. Cicerone (Polymers Division, NIST) 


\section{Chemical Heterogeneity in Chemically Amplified Photoresists: FTIR Measurements and the Effect on Film Dissolution}

The semiconductor industry roadmap indicates that excessive line-edge roughness (LER) in photoresists at sub $45 \mathrm{~nm}$ half pitch is a critical problem with no known manufacturable solutions. It has been broadly accepted that the chemically heterogeneous structure in the photoresist is due to the reaction-diffusion process used to alter the solubility of exposed areas of the material. However, few measurements of this structure have been successful. In this work, we demonstrate that Fourier-Transfer Infrared (FTIR) spectroscopy measurements can be used to parameterize the degree of heterogeneity in polymer thin films, and to illustrate its effect on the response of the polymer to an aqueous base developer solution.

\section{Vivek M. Prabhu}

$\mathrm{T}$ he semiconductor industry roadmap indicates that excessive line-edge roughness (LER) in photoresists at sub $45 \mathrm{~nm}$ half pitch is a critical problem with no known manufacturable solutions. LER is believed to result from the heterogeneous distribution in the deprotection product at the line edge of the latent image. The optical image quality of the exposure and the distribution of the subsequently generated photoacid catalysts is a key factor to LER. This heterogeneous deprotection distribution results in local differences in solubility in the developer solution, where highly deprotected chains dissolve, and those with insufficient deprotection remains in the film. A quantitative measure of the degree of heterogeneity in the deprotection reaction product is needed to identify and enable design strategies to reduce materials sources of LER. Direct experimental observation of the degree of heterogeneity is difficult because of the small size scale, and the limited contrast between protected and deprotected chains.

Here, we developed a method to characterize the degree of heterogeneity in thin polymer films using FTIR on materials that exhibit hydrogen bonding. Carboxyl groups are well known to form dimers that induce a band shift in $\mathrm{C}=\mathrm{O}$ IR spectra relative to the monomer state. Since hydrogen bonding (dimer) can only occur with adjacent $\mathrm{C}=\mathrm{O}$ groups, and the films are processed well below the glass transition, the local distribution in chemical composition generated by deprotection reaction can be deduced from the ratio of hydrogen bonded to free $\mathrm{C}=\mathrm{O}$ groups. As a reference point, we use the fraction of hydrogen-bonded $\mathrm{C}=\mathrm{O}$ groups when the sites are randomly distributed. If the measured fraction exceeds this limit, dimer clusters are expected and the positive deviation from the homogeneous limit is indicative of the degree of heterogeneity. These results can help understand correlations between compositional heterogeneity and the roughness observed upon the dissolution (development) of resists in aqueous bases.
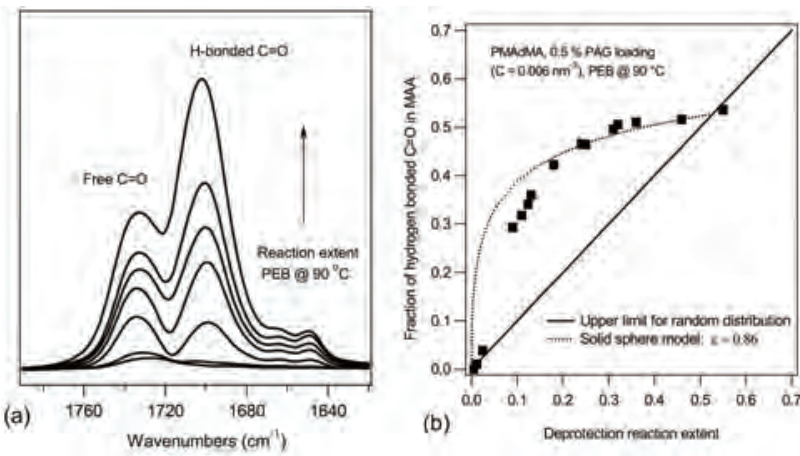

Figure 1: (a) Evolution of $C=O$ stretching bands of $M A A$ groups with reaction extent in PMAdMA samples. (b) Change of fraction of hydrogen bonded $C=O$ in MAA groups with deprotection reaction extent.

Figure 1a shows the IR spectrum of methacrylic acid (MAA) in a partially deprotected poly(methyladamantyl methacrylate) (PMAdMA) film with different extents of reaction. If we define a ratio of the hydrogen bonded $\mathrm{C}=\mathrm{O}$ to the total $\mathrm{C}=\mathrm{O}$ in MAA, $\mathrm{F}_{\text {bond }}$, we can obtain a continuous change of this ratio with the extent of reaction of the film (Figure 1b). Here, $\mathrm{F}_{\text {bond }}$ is calculated from the area under the IR peaks corresponding to hydrogen bonded and free $\mathrm{C}=\mathrm{O}$, respectively. The degree of heterogeneity within the film is defined relative to the ideal homogeneous structure with a random distribution of components.

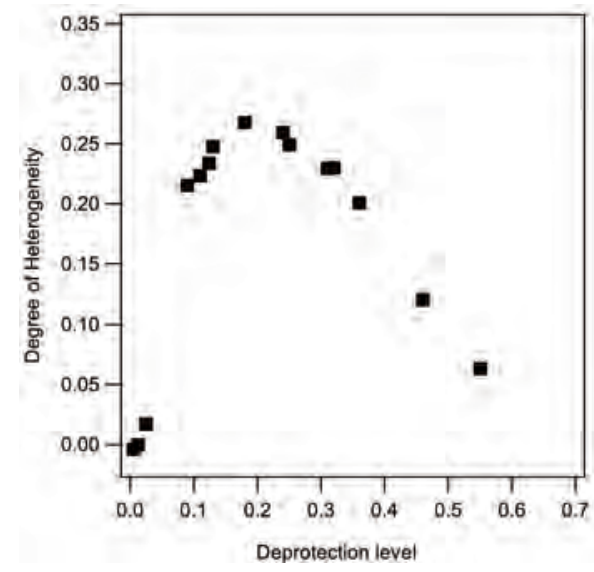

Figure 2: Degree of heterogeneity vs. deperotection level in PMAdMA film for photoacid concentration $C=0.006 \mathrm{~nm}^{-3}$ 
We calculate the degree of heterogeneity $(\mathrm{DH})$ and the dependence of $\mathrm{DH}$ on deprotection level as shown in Fig. 2. It can be seen that the $D H$ is small at very low and very high deprotection level, but is maximized at a deprotection level of approximately 0.20 to 0.25 . These trends arise because the system heterogeneity is dominated by one component at low or high deprotection levels, and the contrast from the uniform phase is small.

The heterogeneity measured from FTIR strongly affects the response of a thin film to an aqueous base solution as a function of the average composition of the polymer as it changes during an acid catalyzed deprotection reaction.

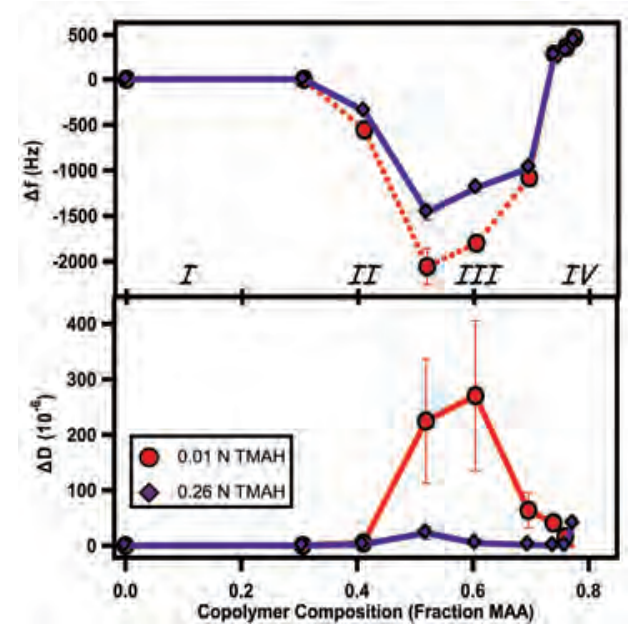

Figure 3: Polyelectrolyte quasi-equilibrium phase behavior determined from the long-time $Q C M$ response in frequency $(\Delta F)$ and dissipation $(\Delta D)$.

Quartz crystal microbalance (QCM) with energy dissipation measurements are used to monitor changes in the crystal resonance frequency (film swelling) and its dissipation (related to its mechanical response). Four different regimes of film behavior were identified when equilibrated with aqueous hydroxide solutions (Figure $3)$. In Regime I, there is no film swelling between compositions $\left(0<f_{\text {MAA }}<0.3\right)$; at higher average degrees of protection $\left(0.3<f_{\mathrm{MAA}}<0.5\right)$, Regime II is characterized by film swelling only; in Regime III

$\left(0.5<f_{\text {MAA }}<0.7\right)$, partial dissolution of a swollen film is observed; and finally, in Regime IV ( $\left.f_{\text {MAA }}>0.7\right)$, nearly complete dissolution is observed.

The different responses of the films to the aqueous solution are a function of the copolymer composition and the distribution of the methacrylic acid within the polymer films (Figure 4). At short reaction (bake) times, the photoacid diffuses and reacts to a limited extent, forming domains of reacted photoresist that do not overlap. At this early stage, Regime I, the hydrophobic continuum of protected polymer, prevents penetration of water. The methacrylic acid content is inaccessible to the solution. With longer reaction time, diffusion and reaction of the photoacid molecules increases the methacrylic acid content, which eventually forms a connected or percolated network. The percolated character of these groups provides pathways for solute transport throughout the film, enabling the hydroxide ions to induce film swelling by titrating methacrylic acid groups. The film swelling is enabled by the percolated distribution of deprotection domains, while the degree of swelling is controlled by the ionizable group content.

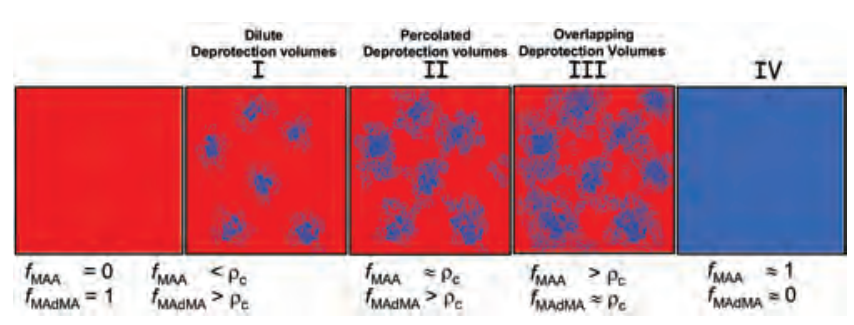

Figure 4: Schematic of the deprotection morphologies of the four regimes and relationship to the average $f_{\text {MAA }}$ (and $f_{\text {MADMA }}$ ) and percolation threshold $\left(\rho_{c}\right)$.

These data raise an interesting question about the origin of the stability of the swollen films. For most polymer gels, film stability during swelling is due to the existence of cross-link junctions formed covalently or by physical entanglement of high molar mass chains. However, the system here does not have any chemical cross linking, and the molar mass of the polymers is below the entanglement molar mass. The stability of our swollen films is attributed to physico-chemical junctions formed by the continuum of hydrophobic (methyladamantyl) groups. The formation of these junctions is analogous to the aggregation of hydrophobic/hydrophilic copolymers in aqueous solution.

The combination of FTIR and QCM measurements provides new insights into the mechanisms of development which are being incorporated into photoresist models of latent-image formation and dissolution. In future work, we plan to extend these results to the gradient latent image line-edge to understand the polyelectrolyte effects at the liquid-photoresist interface by in situ neutron reflectivity.

\section{For More Information on This Topic}

B.D. Vogt, S. Kang, V.M. Prabhu, E.K. Lin, S.K. Satija, $\mathrm{K}$. Turnquest, and W. Wu, Macromolecules 39, 8311 (2006).

A. Rao, S. Kang , B. D. Vogt , V. M. Prabhu, E. K. Lin, W. L. Wu, M. Muthukumar, W. D. Hinsberg, Langmuir 22, 10009 (2006). 


\section{Combinatorial Platform for Measuring the Effect of Substrate Heterogeneities}

To the developers of thin films for coatings, electronics, and nanotechnology, substrate heterogeneities can be the boon of a chemical pattern that drives self-assembly, or the bane of a defect that causes film fail-ure. We have recently developed a combinatorial method for measuring a key under-examined factor in thin film behavior: the "strength" of substrate chemical heterogeneities. Our approach merges soft-lithography and gradient techniques to design a mi-cropatterned library with gradually diminishing chemical contrast. As we demonstrate for the case of polymer film dewetting, our graded test patterns illuminates functional ranges and critical phenomenon in film behavior.

\section{Michael J. Fasolka and Duangrut Julthongpiput}

Urface chemical heterogeneities are essential to the $S$ performance and behavior of substrate supported polymer films. For example, from a quality control standpoint, substrate chemical heterogeneities can be defects that drive instabilities, and thus failure, in polymer film products such as barrier coatings, electronics packaging, and resist systems. Alternately, patterned chemical heterogeneities on a substrate can be advantageous, since they can template structures in an overlying film for micro- and nano-manufacturing aims.

With respect to both of these themes, a critical parameter is the strength of heterogeneity, i.e., the magnitude of chemical differences expressed by the substrate. However, the effect of this factor is difficult to quantify and examine in a methodical manner. In response to this need, we have developed a breakthrough combinatorial library for measuring the effect of differences in surface chemistry, or surface energy.

Our combinatorial test substrates consist of a pattern of micrometer-scale lines that exhibits a continuous gradient in surface energy differences against a constant surface-energy matrix (Fig. 1). The library is fabricated through a vapor-mediated soft lithography of an octyldimethylchlorosilane self assembled monolayer (SAM), which is then treated with a graded ultraviolet (UV)-ozonolysis. The substrate matrix material is SiO2. The library design includes two calibration strips that express the changing and static surface energy of the SAM pattern lines and matrix, respectively. The surface energy contrast, $\Delta \gamma=\gamma_{\mathrm{SiO} 2}-\gamma_{\mathrm{SAM}}$, along the pat-

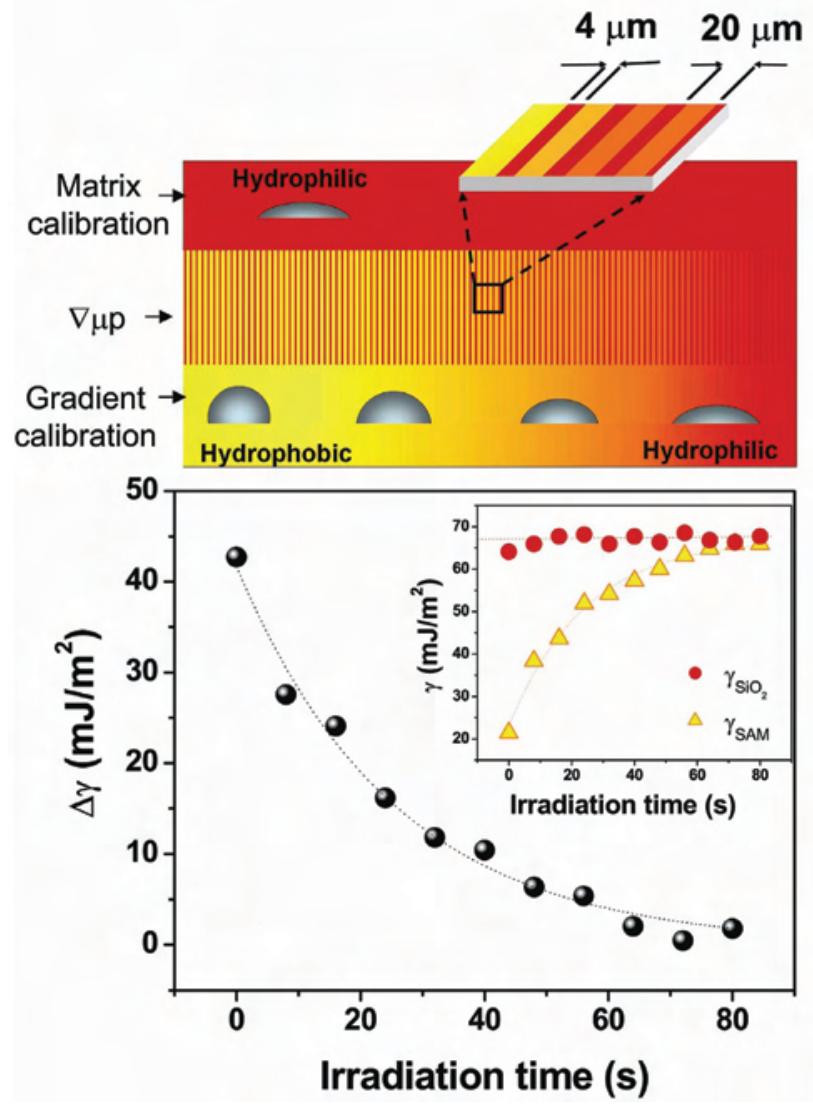

Figure 1: Schematic illustration (top) and calibration (bottom) of the gradient micropattern $(\Delta \mu p)$ library of surface chemical heterogeneities. The inset graph shows $\gamma$ data from the calibration regions.

terned region can thus be determined by contact angle measurements along these calibration regions. In these libraries, $\Delta \gamma$ diminishes from $\approx 45 \mathrm{~mJ} / \mathrm{m}^{2}$ to $0 \mathrm{~mJ} / \mathrm{m}^{2}$ (i.e., a homogeneous surface).

Previously we described how gradient micropattern $(\Delta \mu \mathrm{p})$ libraries can be used to quantify emerging nanometrologies, such as chemical force microscopy [Nanoletters 5, 1535 (2005)]. This year, we illustrated the utility of $\Delta \mu \mathrm{p}$ libraries for mapping thin film phenomenon. As a demonstration case, we examined the dewetting of a polystyrene (PS) deposited on the micropattern. The film was $35 \mathrm{~nm}$ thick and annealed at $60{ }^{\circ} \mathrm{C}$ for $12 \mathrm{~h}$.

As summarized in Fig. 2, the $\Delta \mu$ p enables systematic evaluation of the film dewetting as $\Delta \gamma$ diminishes, and illuminates critical surface energy conditions associated with this behavior. The top of Fig. 2 shows a mosaic of optical micrographs used to assess the dewetted film morphology across the library. An automated optical 
microscope was used to capture this fully contiguous set of images. Figure 2a-d shows four strips of optical microscopy data taken from this array. The top and bottom of each strip respectively show the matrix and SAM calibration regions. As expected from homogeneous areas, the dewetted film exhibits polygonal arrangements of droplets, the scale of which remains constant along the matrix, but which increases as $\gamma$ decreases along the SAM region.

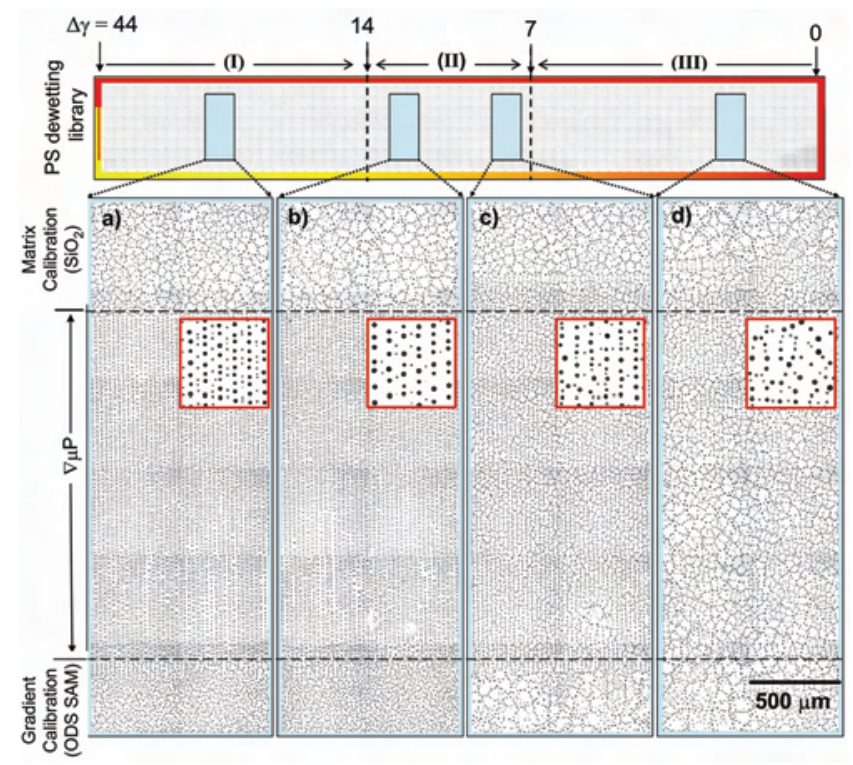

Figure 2: PS film dewetting from a $\Delta \mu$ p library. Top: Contiguous optical micrographs over the entire library. Bottom (a-d): images detailing the transition from pattern-directed to isotropic dewetting, delineated as Regions I-III. Red insets are $250 \mu \mathrm{mx}$ $250 \mu \mathrm{m}$. (ODS SAM=n-octyldimethylchlorosilane self-assembled monolayer)

Middle sections of Fig. 2a-d show dewetting along the $\Delta \mu$ p. Highlight images (red border) detail representative droplet morphologies in each strip. The $\Delta \mu$ p library reveals three regimes of behavior. The borders of these regimes can be delineated with precise $\Delta \gamma$ . values because the library is calibrated, and due to the detail provided by comprehensive optical microscopy measurements.

In Region I, where $\Delta \gamma>14 \mathrm{~mJ} / \mathrm{m}^{2}$, we observe a pattern-directed dewetting. Region I is characterized by ordered arrays of PS droplets which are distributed along the center of both the matrix and SAM portions of the pattern. In Region III, where $\Delta \gamma<7 \mathrm{~mJ} / \mathrm{m}^{2}$, isotropic dewetting occurs. In this region, film dewetting is not affected by the substrate pattern, and the droplet morphology closely resembles the polygonal arrangements observed in the calibration regions. A crossover between these limiting behaviors is exhibited in Region II $\left(7 \mathrm{~mJ} / \mathrm{m}^{2}<\Delta \gamma<14 \mathrm{~mJ} / \mathrm{m}^{2}\right)$ where droplets gradually lose registry with the underlying pattern. More detailed analysis in this region (not shown) indicates

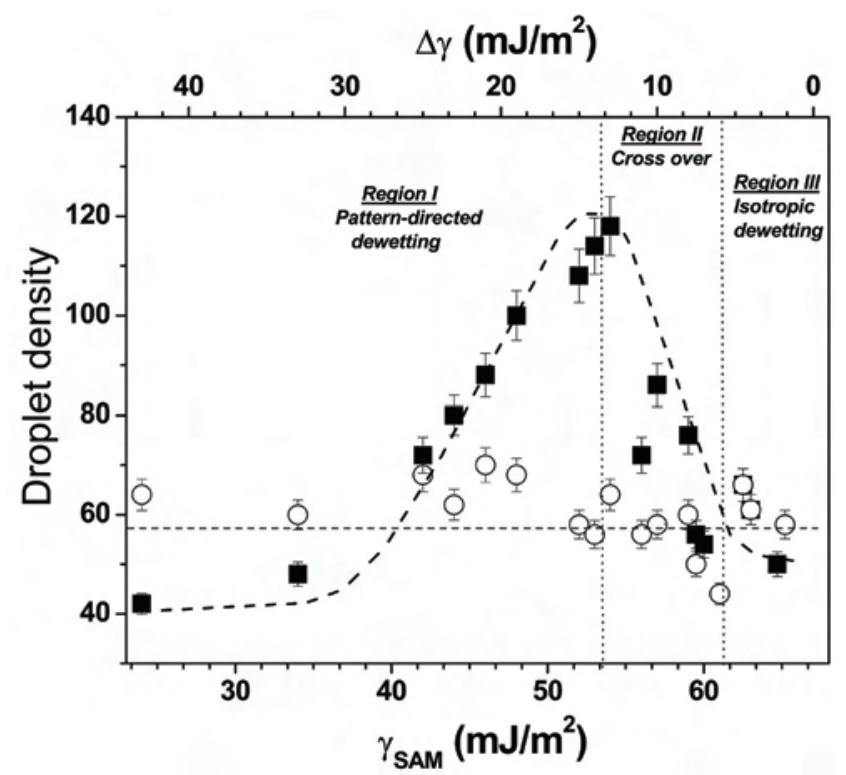

Figure 3: Summary of droplet density data derived from images in Figure 2. Three regimes of droplet behavior are indicated in the figure.

that this crossover is largely due to competition between the kinetics of isotropic and pattern-driven dewetting. By comprehensively mapping dewetting behavior, the $\Delta \mu$ illuminates functional ranges and critical values of substrate chemical heterogeneity for film applications. For example, the library shows that for self-assembly of PS droplet arrays, the optimum pattern strength is in the range of $14 \mathrm{~mJ} / \mathrm{m}^{2}<\Delta \gamma<20 \mathrm{~mJ} / \mathrm{m}^{2}$. Likewise, to avoid heterogeneity-driven dewetting, surface energy differences on the substrate need to be less than $7 \mathrm{~mJ} / \mathrm{m}^{2}$ in magnitude.

In FY06, we reported this work through an invited lecture at the American Chemical Society fall meeting, and at the 2006 International Waterborne Coatings Symposium.

\section{For More Information on This Topic}

A. Karim, J. Douglas, E. Amis (Polymers Division, NIST). For more information on the NIST Combinatorial Methods Center, see www.nist.gov/combi. 


\section{Comparative Metrologies for Evaluating Single Wall Carbon Nanotube (SWNT) Dispersion}

\begin{abstract}
Despite claims of SWNT dispersion throughout the literature, there is currently no consensus on an absolute measure, nor has there been a methodical comparison of different approaches that might serve as a foundation for such a measure. We demonstrate that small-angle neutron scattering (SANS) provides the most direct measure of dispersion. Near-infrared fluorescence spectroscopy, proposed to be sensitive to the bundling or clustering of SWNTs, similarly exhibits a definitive measure. In contrast, optical absorption spectroscopy and resonant Raman scattering have limited sensitivity. Our study represents the first controlled comparison of currently accepted metrics for evaluating SWNT dispersion, and serves as a useful point of reference for any of the four techniques.
\end{abstract}

\section{Jeffrey A. Fagan and Erik K. Hobbie}

S ingle wall carbon nanotubes (SWNTs) exhibit re$S$ markable physical properties, and have the potential for profound impact over a broad range of technologies, from first generation applications such as conducting and mechanically reinforced plastics, to more advanced applications such as fuel cells, microelectronic components, flat panel displays, and biochemical sensors. In many of these applications, efficient dispersion of SWNTs at the individual nanotube level will be a prerequisite for optimal performance. Sources of imperfection in dispersing individual SWNTs are most likely bundles, defined here as the lengthwise aggregation or "roping" of tubes, and aggregates, the fractal-type networking of tubes in a floc.

Of all the measurement techniques currently used to evaluate SWNT dispersion in solutions and composites, small-angle scattering is perhaps the simplest to interpret and understand. This technique directly probes two-point correlations in composition, and can thus distinguish true form scattering due to individual SWNTs from the structural scattering that arises from nanotube aggregates and bundles. Other more readily available methods, however, have also recently been used to evaluate SWNT dispersion, typically by focusing on subtle effects such as those that tube-tube interactions have on the optical signatures associated with transitions between electronic states in SWNTs of different chirality. Most notably, these techniques include optical absorption spectroscopy, near-infrared (NIR) fluorescence spectroscopy, and resonant Raman scattering. To extract maximum information from these optical techniques, the sensitivity of each to the level of SWNT dispersion must be known, especially as the limit of individually dispersed tubes is approached.

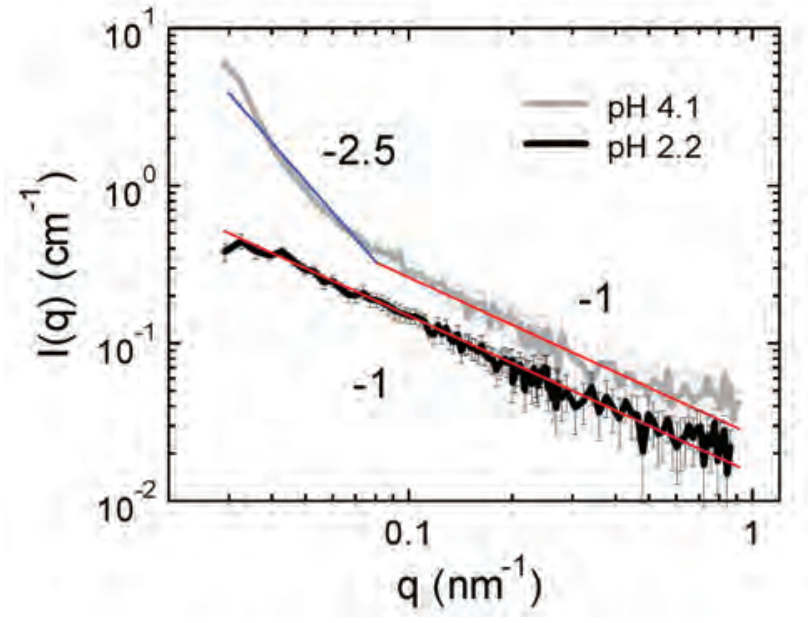

Figure 1: SANS intensity as a function of scattered wave vector for SWNT-PAA composites cast from ssDNA stabilized SWNTs in unbuffered ( $p H$ 2.2) and buffered ( $p H 4$.1) aqueous $240 \mathrm{k}$ $P A A$ solution, where the red lines denote a power law with an exponent of -1 , and the blue line a power law with exponent -2.5 . The black trace has been shifted down slightly for clarity by a constant multiplication factor of 1.77. Error bars denote two standard deviations in the total experimental uncertainty.

Despite claims of SWNT dispersion throughout the literature, there is currently no consensus on an absolute measure, nor has there been a methodical comparison of different approaches that might serve as a foundation for such a measure. For this purpose, we have prepared model polymer composites composed of single-stranded deoxyribonucleic acid (ssDNA) stabilized SWNTs dispersed in polyacrylic acid (PAA) for an evaluation of the different metrologies currently used to assess SWNT dispersion. By varying the $\mathrm{pH}$ of the precursor PAA-SWNT solution, a systematic variation in SWNT aggregate dispersion is evaluated by scattering and optical spectroscopy. Small-angle neutron scattering (SANS) provides the most direct measure of dispersion. Near-infrared fluorescence spectroscopy, proposed to be sensitive to the bundling or clustering of SWNTs, similarly exhibits a definitive measure. In contrast, optical absorption spectroscopy and resonant Raman scattering have limited sensitivity. Our study represents the first controlled comparison of currently accepted metrics for evaluating SWNT dispersion, and serves as a useful point of reference for any of the four techniques.

SANS profiles from the two composite samples are shown in Fig. 1. For fully dispersed linear objects, we would expect a power law of -1 . The unbuffered composite exhibits this power law over the entire q range measured, corresponding to rigid-rod behavior over a range of length scales between (6 and 200) nm. In comparison, the buffered composite exhibits a distinctive 
upturn in scattering at low-q, approximately proportional to a power law with an exponent of -2.5 , which is characteristic of nanotube clustering. The difference is both striking and conspicuous, directly demonstrating ideal nanoscale SWNT dispersion in the unbuffered composite, as well as the ability to tune dispersion through adjusting the $\mathrm{pH}$ of the precursor PAA solution.

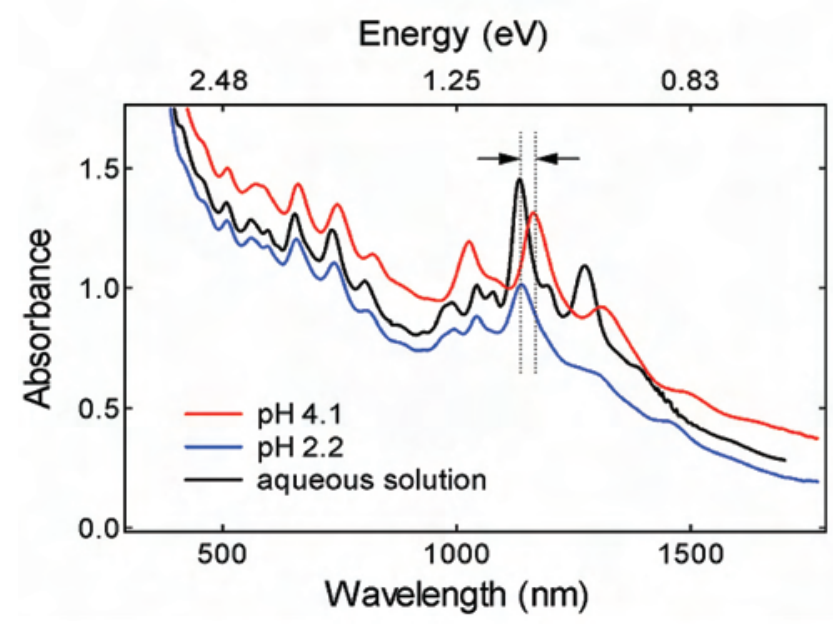

Figure 2: UV-Vis-NIR absorbance as a function of wavelength for the same SWNT-PAA composites, where the solid/black trace denotes the spectra of the same concentration of ssDNA wrapped SWNTs in pure aqueous suspension ( $\mathrm{pH} 7$ ).

Figure 2 shows a comparison of the ultravioletvisible-near-infrared (UV-Vis-NIR) absorption spectra obtained from the two composites, along with that from the initial aqueous ( $\mathrm{pH}$ 7) ssDNA stabilized SWNT suspension. The relative intensity of the peaks corresponding to the excitonic transitions for SWNTs of specific chirality in the NIR region of the spectrum are reduced from aqueous solution to buffered composite to unbuffered composite. The second difference between samples is a redshift in the position of the dominant absorption peak from $1134 \mathrm{~nm}$ in the aqueous solution and unbuffered composite to $1163 \mathrm{~nm}$ in the buffered composite. The changes in the peak structure leading to a broader and redshifted absorption in the buffered sample compared to the unbuffered one supports the SANS results that enhanced dispersion is achieved for the unbuffered composite.

Fluorescence spectroscopy is also a sensitive measure of SWNT dispersion, since SWNT bundling quenches the NIR fluorescence associated with the narrow band gap of the semiconducting SWNT species. A comparison of the fluorescence maps for the unbuffered and buffered PAA composites, shown in Fig. 3, illustrates a pronounced difference in their spectral emissions. In contrast, resonant Raman scattering from the SWNT radial breathing mode (Fig. 4) shows very little difference between the two samples. In combination, these two observations point to fully debundled SWNTs that have aggregated in the $\mathrm{pH} 4.1$ composite.
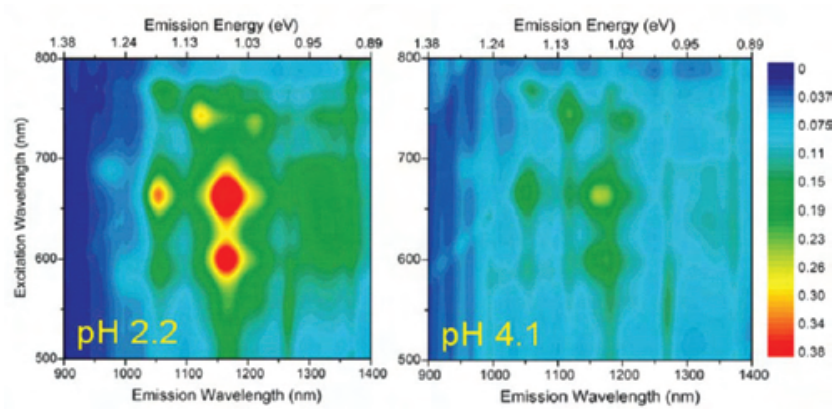

Figure 3: Comparison of NIR fluorescence plots for the SWNT composite samples. Despite equal SWNT concentration and near equal film thicknesses, the fluorescence of the unbuffered film is substantially larger.

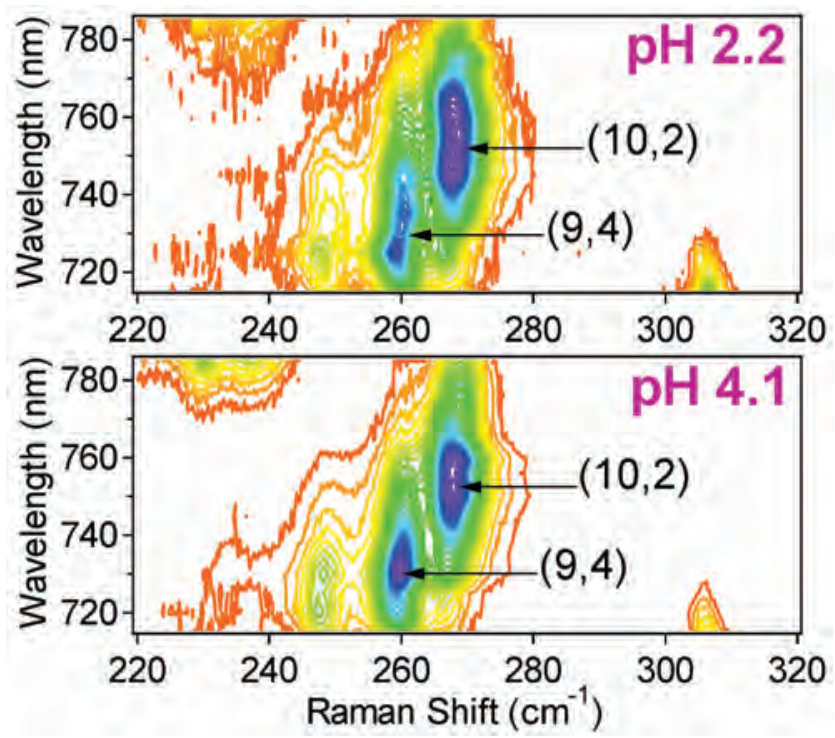

Figure 4: Radial breathing mode resonant Raman scattering for the two SWNT composites.

From this comparison, SANS provides the most direct measure of nanotube dispersion, followed by near-infrared fluorescence spectroscopy. Resonant Raman scattering and optical absorption spectroscopy are useful, but the limits of these techniques for assessing the relative level of dispersion need to be clearly understood, as they provide measures with more subtle changes in signal. From our results, the independent use of any of these techniques can be improved in its interpretation.

\section{For More Information on This Topic}

J.A. Fagan, E.K. Hobbie, K.B. Migler, B.J. Bauer (Polymers Division, NIST) 


\section{Numerical Optimization of Complex Instrumentation}

A novel approach has been created for the selection of optimal instrument parameters that yield a mass spectrum which best replicates the molecular mass distribution of a synthetic polymer. The application of implicit filtering algorithms was shown to be a viable method to find the best instrument settings, while simultaneously minimizing the total number of experiments that need to be performed. This includes considerations of when to halt the iterative optimization process at a point when statistically significant gains can no longer be expected. This work represents part of an effort to develop an absolute molecular mass distribution polymer Standard Reference Material ${ }^{\circledR}$ by matrix-assisted laser desorption/ionization time-offlight (MALDI-TOF) mass spectrometry.

\section{William E. Wallace}

$\mathrm{T}_{\mathrm{p} \text { tas }}$ pical analytical instrumentation optimization is performed by the analyst by simply applying the "factory settings" or by "optimizing by eye." This is because an exhaustive search of the parameter space for modern instrumentation with many adjustable parameters is prohibitively time consuming. However, a variety of little-known mathematical methods exist that enable the experimentalist to optimize instrument settings without performing an exhaustive search. Broadly classified, these methods are all forms of numerical optimization. When the topology of the search space is very complex, for example, when it has great sensitivity to one or more parameters (as mass spectrometers often do), the methods used are part of the field of non-linear programming. They are called non-linear because some (or all) of the instrument parameters do not have a linear relationship between parameter value and measurement response. A simple example is laser intensity in MALDI-TOF mass spectrometry and its effect on signal-to-noise ratio where a relatively sharp threshold is observed experimentally. When the measurement outcomes (which in the present case are mass spectra) contain random noise, the mathematical methods are termed stochastic numerical optimization. Stochastic methods are important in mass spectrometry because all mass spectra have noise, this noise varies as the instrument parameters are adjusted, and the noise will often change across the spectrum. Measurement noise presents a significant challenge to any optimization method especially for cases where signal to noise is not the measurand to be optimized. Nevertheless, numerical optimization methods offer experimentalists a way to tune the instrument parameters to achieve the desired goal without having to search all possible parameter combinations. To measure the absolute molecular mass distribution of a synthetic polymer, it would be ideal to locate a region in parameter space where the instrument response function was uniform across the entire mass range. Finding the instrument response function is necessary to calibrate the intensity axis of the mass spectrum; that is, to go from mass spectrum to molecular mass distribution. If the instrument response function is uniform, then the relative peak areas in the mass spectrum correspond directly to the relative abundances of individual n-mers in the sample. A uniform instrument response function would be a line of zero slope; that is, it would have a derivative of zero. If not uniform, the instrument response function could slowly vary across the mass range, preferably linearly with mass. The optimal conditions are those that give the simplest (or flattest) instrument response function; that is, the one with the smallest derivative.

To measure the instrument response function, a gravimetric mixture was made of three low polydispersity polystyrenes that were very close in average molecular mass. The optimal instrument settings were those that provided the closest match between the total integrated peak intensity of each of the three polymers with the known gravimetric ratios. Note that there is no guarantee (or even assumption) that the optimal instrument settings that give the flattest instrument response function will also yield optimal signal to noise ratios. In fact there is no reason to believe that a search for the instrument settings that optimize the response function will not lead into a region where the mass spectra become so noisy as to make peak integration impossible. Thus, to find the optimal instrument settings, we used stochastic gradient approximation methods. These methods have proven to be extremely robust in cases where the measured data are very noisy.

Optimization is performed by defining an objective function $J(x)$ where $x$ is a vector consisting of the instrument parameters. In our case, the objective function was the sum of the squared differences between the amount of each polymer in a mixture created gravimetrically, and the amount of each polymer in the mixture found by mass spectrometry. When this function is zero, the gravimetric concentrations match the concentrations found by mass spectrometry, and the instrument is optimized. The function $J(x)$ is a noisy function with respect to the parameter vector $\mathrm{x}$, due to the inherent statistical noise in the mass spectra. This complicates the task of numerically locating the minimum of $J(x)$. The fact that each evaluation of $J(x)$ requires an experiment, and subsequent interpretation of experimental 
results, means that there is a high cost for each function evaluation. This further complicates any numerical procedure that seeks to minimize $J(x)$. Finally, there are values of the vector $x$ (for example, out of range instrument parameter settings), for which $J(x)$ cannot be evaluated. One method for minimizing noisy functions that seeks to approximate the gradient of the objective function is called implicit filtering. Broadly speaking, this method uses a very coarse grained step-length to build a finite difference approximation to the gradient of $J(x)$. This gradient is then used to generate steep-descent directions for a minimization process. As iterates draw closer to the solution, and the objective function decreases, the finite difference step-length is decreased until it approaches a number small enough to suggest convergence of the algorithm to the minimum value.

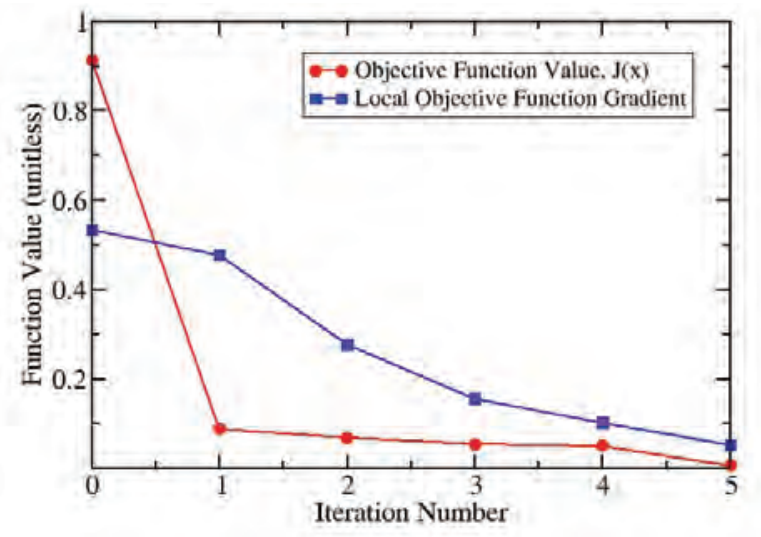

Figure 1: The objective function $J(x)$ and its local gradient value as a function of iteration step.

In Fig. 1, there is an initial steep drop in the objective function flowed by gradual movement to the optimal parameter settings. The gradient of the objective function also decreases steadily as the optimum point is approached. These monotonic responses indicate that the optimization routine is stable. At the optimum value, the objective function is so small that it cannot be reduced further due to the inherent noise in the measurement. Likewise, the step size indicated for each parameter at this point is so small as to be below the precision of the instrument's settings.

In Fig. 2, the values oscillate about their final values as the optimization proceeds. The laser intensity undergoes the greatest excursions: decreasing in the first two iterations, returning to its initial value in the third iteration, and then increasing in the fourth iteration before settling into its final value. The four other parameters make an excursion in the direction of their final values in the first iteration, return to their initial value in the second iteration, and find the equilibrium values by the third iteration. This zigzag pattern is characteristic of the non-linearity of the system. This non-linearity arises from the fact that the instrument parameters are coupled; that is, varying one requires all others to vary in response if $J(x)$ is to move closer to its optimal value. Thus, the vector $x_{i+1}$ has a tendency to be normal to the vector $x_{\mathrm{i}}$ (in its five-dimensional space). The laser intensity varies the most, and would seem to be the dominant variable. It seeks its stable value before the other parameters can settle down to find their optimal values.

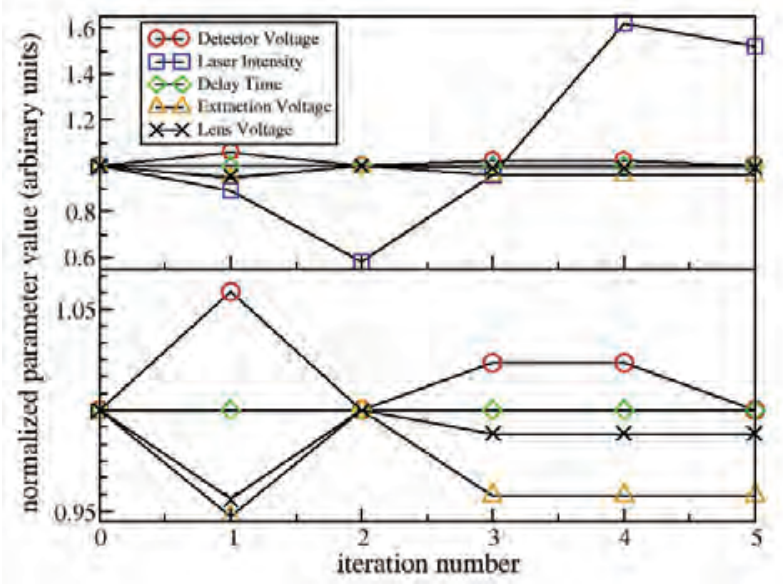

Figure 2: Individual instrument parameter values as a function of iteration number.

A specialized noise-adapted filtering method has been applied to the problem of finding the optimal instrument parameters for a MALDI-TOF mass spectrometer. Finding the optimal instrument parameters was a critical step in creating an absolute molecular mass distribution polymer Standard Reference Material ${ }^{\circledR}$. The task of tuning the instrument's five main parameters could not be approached by exhaustive search methods, given the amount of effort needed to take and to reduce the data in a statistically meaningful way at each set of instrument parameters. Additionally, this method produces an estimate of the sensitivity of each optimal parameter not available to traditional exhaustive search methods. Each of the subtasks in the process could be automated to create an integrated closed-loop optimization scheme.

\section{For More Information on This Topic}

W.E. Wallace, K.M. Flynn, C.M. Guttman (Polymers Division, NIST); A.J. Kearsley (Mathematical and Computational Sciences Division, NIST)

See www.nist.gov/maldi for more information. 


\section{Platform for Characterizing Defects in Magnetic Nanoparticles}

\begin{abstract}
Nanoparticles are an important component of emerging technologies for medical diagnostics, targeted therapies, biomolecular detection, and next generation electronics. The efficacy of nanoparticles in these applications is challenged by the lack of methods to characterize the strength and homogeneity of nanoparticle interactions. We are developing a measurement platform to rapidly quantify nanoparticle interactions for large assemblies of nanoparticles, providing statistics on the distribution in strength of interaction, and the capability to determine the nature and concentration of defective particles.
\end{abstract}

\section{Ronald Jones and Jason Benkoski}

$\mathrm{N}$ anoparticles with diameters of (1 to 100) nm are widely sought as enablers of advanced technologies for medical diagnostics and therapies, chemical and biological sensors, and microelectronics. Many of these technologies rely on the enhanced optical, magnetic, and chemical functionality inherent in particles of nanometer scale. However, properties of nanoparticles are highly sensitive to particle size, shape, and chemical composition. As a result, small variations in the manufacturing process can produce defective particles with undesirable properties, resulting in a loss of device functionality. The advancement of suitable manufacturing techniques is currently limited by a lack of measurement platforms capable of rapidly characterizing defects in nanoparticle interactions over large particle quantities.

A specific class of nanoparticles that has received significant interest is magnetic nanoparticles (MNPs). The particles typically consist of a metallic core with a polymeric flocculant coating. Recent advances in synthetic methods make the production of large numbers of MNPs with nearly monodisperse diameters and homogeneous compositions feasible. However, scaling established methods to characterize the interaction strength of a single MNP are challenged by the large numbers of particles and their small dimensions, precluding a measurement of defect concentration and homogeneity in manufacturing. As a result, indirect and often timeconsuming measurements of size dispersity are used to infer homogeneity of interactions.

We are developing a methodology to rapidly characterize the homogeneity of large numbers of particles to quantify the number density of defective particles. Defective particles are particles with vanishing magnetic moment or particles with undesirable polarity. In many applications, for example, the synthesis attempts to cre- ate large batches of dipolar particles. However, small variations in synthetic conditions can result in varying concentrations of quadripolar particles, or even particles with higher moments of polarity.

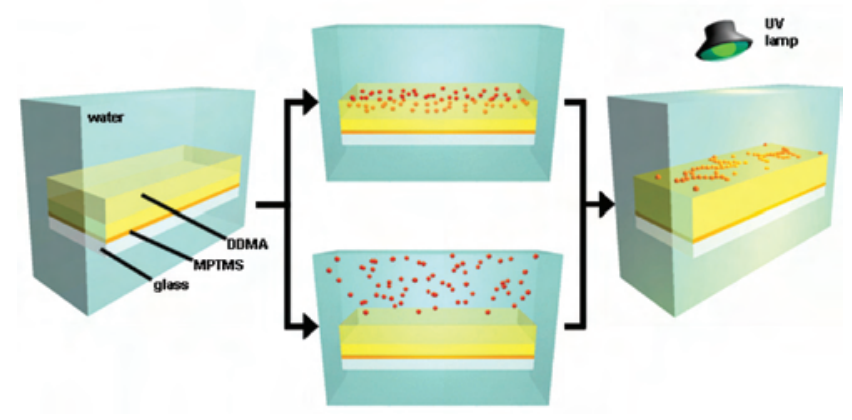

Figure 1: Schematic depicting the crosslinkable oil-water platform for characterizing nanoparticle interactions. The platform provides a rapid method to quantify the character and number of defects in magnetic interactions for a large number of particles. A wide range of particle size and chemistry are available to this methodology due to the ability to assemble oil soluble (top route) and/or water soluble (bottom route) particles.

Rather than measuring the properties of individual particles in a serial fashion, our methodology measures the shape and size of nanoparticle assemblies, where assemblies can consist of 10's or 100's of nanoparticles. Dipolar MNPs are known to assemble as long strings in solution when their magnetic moment interactions exceed other isotropic forces, including thermally induced vibrations and isotropic van der Waals interactions. In contrast, non-polar particles or multipolar particles form non-linear assemblies. Defects in MNPs are therefore characterized by the linearity within assemblies. The methodology described here uses a novel platform to freeze-in thousands of assemblies for characterization using scanning probe or electron microscopies. By measuring a large number of assemblies, where each assembly can contain 1000's of particles, high precision statistics of defect concentration are obtained.

As shown in Fig. 1, MNP assembly occurs at an oil/ water interface, in which the oil phase can be flashcured upon ultraviolet (UV) radiation exposure. The platform utilizes a photopolymerizable oil phase, 1,12-dodecanediol dimethacrylate (DDMA), to provide a portable and characterizable "snapshot" of the assembly process. The use of a low viscosity oil and water phase allows MNP assembly to proceed rapidly. With an appropriate chemistry and light source, the assemblies are frozen in less than $1 \mathrm{~s}$ from initial exposure, with minimal volume change of the oil phase. Freezing of the MNP assemblies precludes the alteration of as- 
sembly structure by capillary forces at the air/water interface. The resulting sample is glassy, allowing storage for long-time or future measurements, and is amenable to a wide range of characterization platforms, including scanning probe and electron microscopies. Its transparency to a wide range of electromagnetic wavelengths will enable the advancement of scattering and optical imaging based characterization platforms.

While development has focused on nanoparticles, a wider range of particle interactions can be studied under identical conditions, including much larger colloidal and latex particles, particles of varying polarity, and of varying composition, including metallic and polymeric. Particles investigated to date include nonpolar $0.39 \mu \mathrm{m}$ poly(methyl methacrylate) latex spheres, nonpolar $10 \mu \mathrm{m}$ polystyrene latex spheres, highly polarizable $5 \mathrm{~nm}$ Au nanocrystals, dipolar $10 \mathrm{~nm}$ CdTe quantum dots, and magnetic $25 \mathrm{~nm}$ magnetite nanoparticles. As shown in Fig. 2, the range of structures expected in these assemblies is large, making the methodology generalizable to a large range of particles.

In Fig. 3, assemblies of both micrometer scale polymer particles and nanometer scale metallic particles provide immediate qualitative and quantitative characterizations of average interaction strength, while providing data on defect concentrations. Specifically, charging on polystyrene particles as a result of the sample preparation method dramatically changes the assembled structure from dense, two-dimensional aggregates to highly branched networks. The charging appears to be uniformly applied across all particles. In contrast, gold nanoparticles follow a well defined random walk characteristic of directional forces. Similarly sized magnetite particles feature regions of dense packing, indicating the presence of particles with lower magnetic moment and/ or multipolar character.

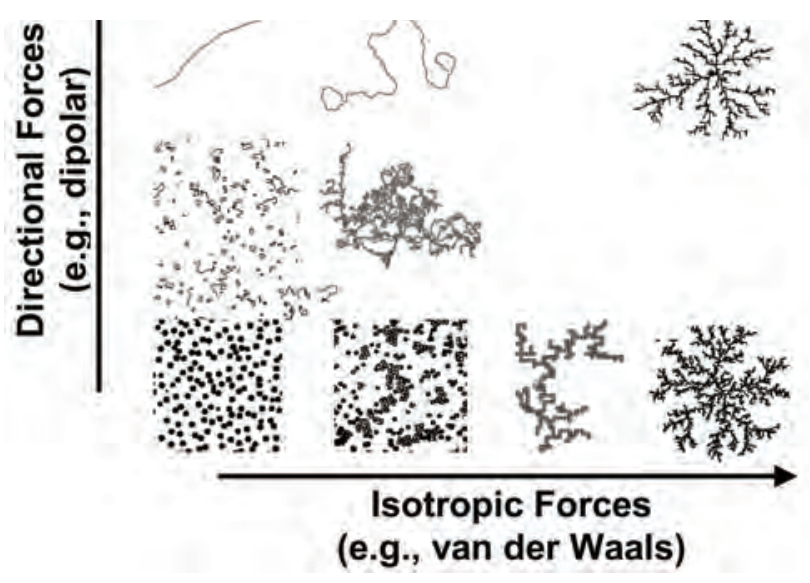

Figure 2: Representative structures from assemblies of particles with varying types and strength of interaction.

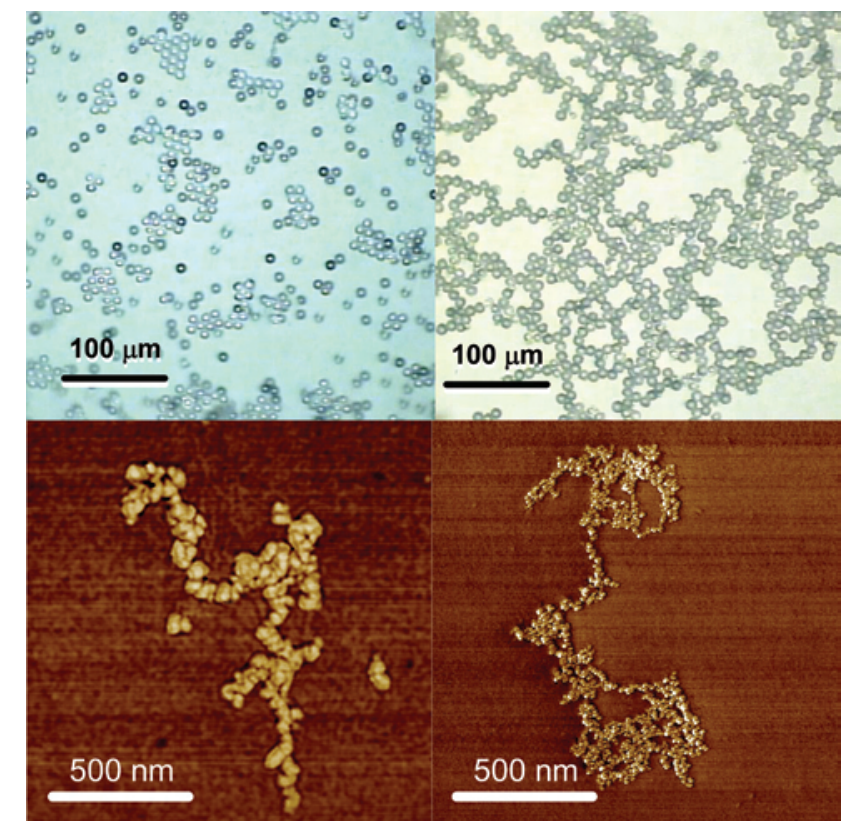

Figure 4: Top: Optical micrographs of $10 \mu \mathrm{m}$ PS spheres protruding from the oil/water interface after crosslinking. Shown are uncharged particles (left) with characteristic two-dimensional dense assemblies. In contrast, a small charge added to the particle creates a highly branched assembly (right). Bottom: Atomic force microscope phase image of assemblies of gold (left) and magnetite (right) nanoparticle assemblies. While the gold particles show a characteristic linear assembly, the presence of dense assembled regions in the magnetite particles may indicate the presence of particles with reduced magnetic moment.

We have demonstrated the use of a DDMA/water interface as a platform for characterizing defects in the magnetic interactions of particles with nanometer scale dimensions. The methodology characterizes the interaction strength of a large number of particles by the examining the structure of assemblies of 10's or 100's of nanoparticles at an oil/water interface. Without the ability to preserve snapshots of the aggregated structures by this process, the rapid movement of the particles would challenge microscopy characterization techniques. The high interfacial tension of the oil/water interface provides a strong driving force for the segregation of many different particle types, despite large differences in charge, polarizability, size, and surface chemistry. It therefore allows one to compare the aggregation of a wide variety of nanoparticles under remarkably similar conditions.

\section{For More Information on This Topic}

J. Douglas, A. Karim (Polymers Division, NIST); J. Pyun (University of Arizona) 



\section{Advanced Manufacturing Processes}

The competitiveness of U.S. manufacturers depends substantially on their ability to create new product concepts and to quickly translate such concepts into manufactured products that meet their customers' increasing expectations of performance, cost, and reliability. This is equally true for well-established "commodity" industries, such as automotive, aerospace, and electronics; for materials suppliers of aluminum, steel, and polymers; and for rapidly growing industries based on nanotechnology and biotechnology. In support of these industries, the NIST Materials Science and Engineering Laboratory (MSEL) is developing robust measurement methods, standards, software, and process and materials data needed for design, monitoring, and control of new and existing materials and their manufacturing processes.

Our research is conducted in close collaboration with industrial partners, including industrial consortia, and with national standards organizations. These collaborations not only ensure the relevance of our research, but also promote rapid transfer and utilization of our research by our partners.

Contact: Michael J. Fasolka

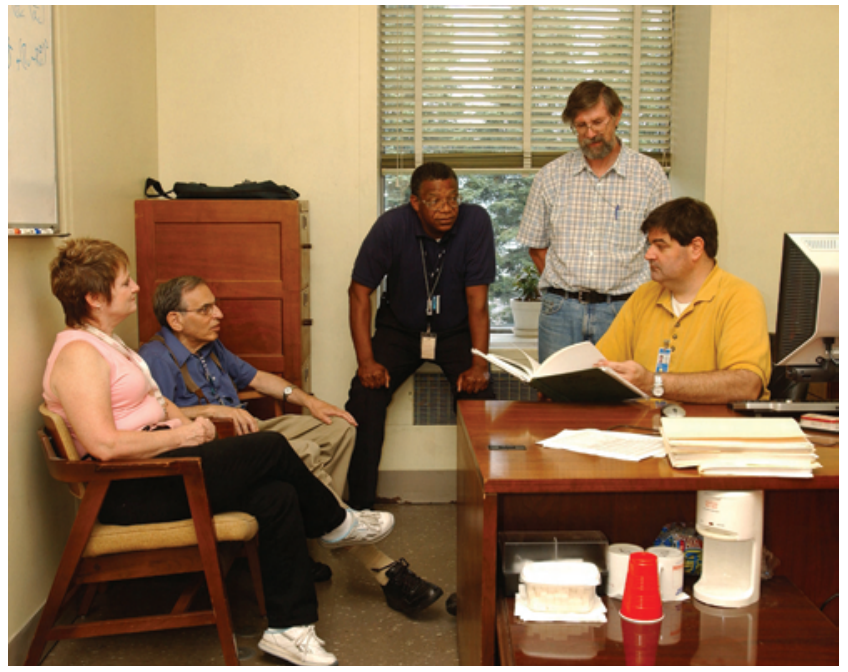

Quantitative Polymer Mass Spectrometry Project Meeting

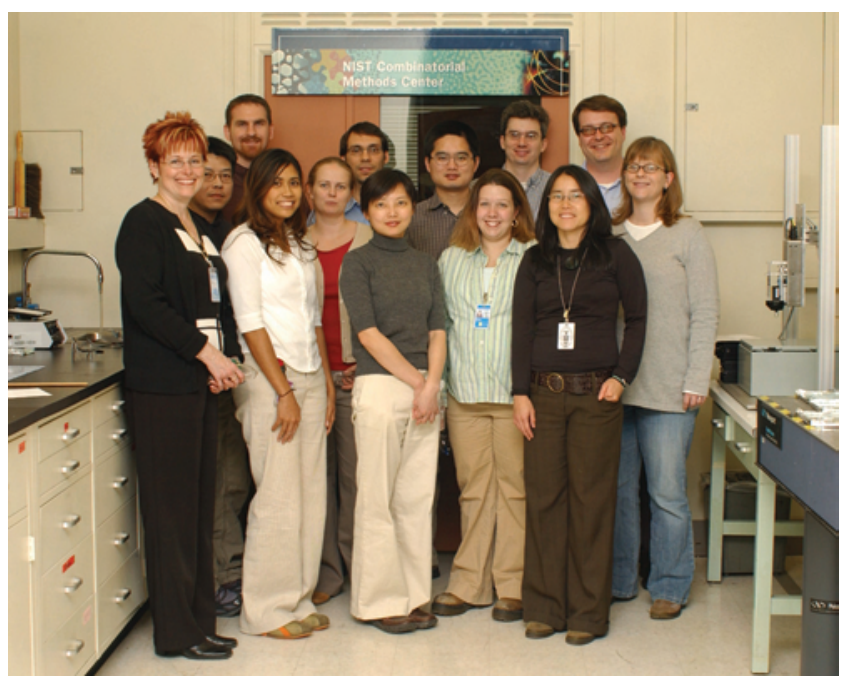

NIST Combinatorial Methods Center 


\section{NIST Combinatorial Methods Center}

\section{A Pioneer and Partner in Accelerated Materials Research}

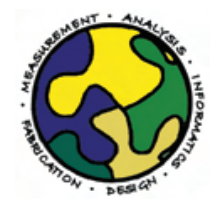

Combinatorial and high-throughput (C\&HT) methods hold great potential for making materials research more productive, more thorough, and less wasteful. However, significant technical barriers prevent the widespread adoption of these revolutionary approaches by industry. Through creative, cost-effective measurement solutions, and by using a customer outreach model designed for fruitful collaboration, the NIST Combinatorial Methods Center (NCMC) strives to ease the acquisition of C\&HT techniques by the materials research community.

\section{Michael J. Fasolka and Carol E. Laumeier}

$\mathrm{T}$ he NIST Combinatorial Methods Center (NCMC), now in its fifth year, continues to be an innovative resource for industrial materials researchers striving to acquire combinatorial and high-throughput (C\&HT) measurement capabilities. The NCMC fosters adaptation of C\&HT methodologies through two complementary efforts. First is a research program, centered in the Polymers Division's Combinatorial Methods Group, which develops and demonstrates C\&HT measurement solutions for the discovery and optimization of complex materials products, including polymer coatings and films, adhesives, cosmetics, and structural plastics. Moreover, in conjunction with other Polymers Division Groups, a growing aspect of NCMC research provides C\&HT methods for emerging technologies such as nanostructured materials, organic electronics, and biomaterials. All NCMC research is published in the public domain.

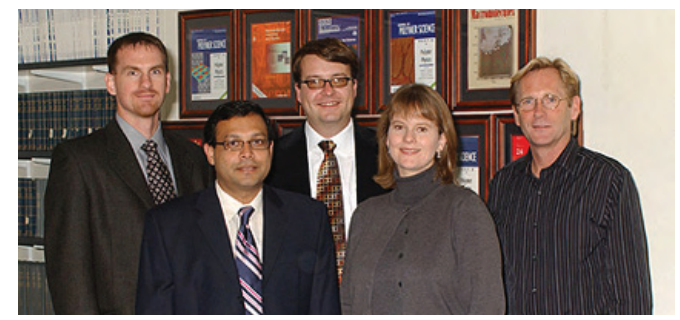

Figure 1: NCMC Awardees of the Department of Commerce Silver Medal for Customer Service in 2006. From left to right: C.M. Stafford, A. Karim, M.J. Fasolka, K.L. Beers, E.J. Amis.

Second, to complement its research, the NCMC conducts outreach efforts focused on disseminating new measurement methods to industry, assessing emerging industrial C\&HT metrology needs, and forming a community of technology stakeholders. The NCMC leverages a unique, "non-proprietary" consortium model, the development and success of which garnered a Department of Commerce Silver Medal for Customer Service (see photo). This model promotes open discus- sion of the metrology issues that face industrial stakeholders, and a focus on the fundamental, common needs of this community. NCMC consortium members (see table below) are served through a series of semi-annual workshops, a dedicated website, and documents that detail NCMC instrumentation. FY06 activities included two additions to the NCMC workshop series. NCMC-8: Polymer Formulations II, held Oct. 27-28, 2005, assessed opportunities in microscale measurement technologies for advancing formulations science, gauged related measurement needs, and reported the achievements of the Polymers Formulations project. NCMC-9: Combinatorial Methods for Nanostructured Materials, held Apr. 24-25, 2006, determined key metrology needs in industrial research of nanostructured polymeric materials, and set priorities for developing C\&HT methods for these systems. NCMC-9's Workshop Report, NISTIR 7332, is available on the NCMC website (see below).

In addition, non-proprietary "Focus Projects" enable member companies to collaborate with NCMC researchers to produce more specific measurement solutions. Two NCMC Focus Projects were completed in 2006. The first, co-funded by Procter and Gamble and National Starch, resulted in a powerful microfluidic approach for measuring interfacial tension. The second, sponsored by Intel and National Starch, resulted in combinatorial libraries that assess the performance of epoxy adhesives for flip-chip electronics. A new Focus Project, with the goal of developing HT measurements of polymer hydrogel mechanics, was launched with Vistakon, a manufacturer of contact lenses.

$\begin{array}{lr}\text { NCMC Members } & \left({ }^{*} \text { New in FY2006) }\right. \\ \text { Air Products \& Chem. } & \text { Insight Faraday* } \\ \text { Arkema Inc. } & \text { Intel } \\ \text { Avon* } & \text { L'Oreal } \\ \text { BASF } & \text { PPG Industries } \\ \text { Bayer Polymers } & \text { Procter \& Gamble } \\ \text { Dow Chemical Company } & \text { Rhodia } \\ \text { ExxonMobil Research } & \text { Symyx Technologies* } \\ \text { Honeywell International } & \text { Unilever* } \\ \text { Hysitron International } & \text { Univ. of Southern Miss. } \\ \text { ICI/National Starch \& Chem. } & \text { Veeco Metrology }\end{array}$

For more information, please visit the NCMC website at www.nist.gov/combi.

\section{Contributors and Collaborators}

E.J. Amis, K.L. Beers, A. Karim, C.M. Stafford (Polymers Division, NIST) 


\section{Polymer Formulations:}

\section{Soft Materials Measurements on a Chip}

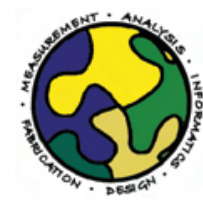

Complex structured fluids, also known as formulations, have been an important part of modern life for many years, even though they are poorly understood. Only recently has industry begun to control and design these materials to obtain novel, valuable properties, but scientists are overwhelmed by the composition and processing variable space required. We develop and demonstrate high throughput methods to advance polymer formulations science through the fabrication of microscale instrumentation for measuring physical properties of complex mixtures.

\section{Kathryn L. Beers}

Significant progress was made this year in our $\checkmark$ efforts to build microfluidic instrumentation for the measurement of polymer solutions. New measurement capabilities were developed, several collaborators visited NIST for extended periods to learn new technologies, and high impact publications resulted from our work.

Dynamic light scattering (DLS) remains one of the most popular and powerful tools to measure size in solutions containing nanoparticles (such as latexes and suspended inorganics) and nanostructures (such as micelles and vesicles). Several prototypes were constructed that enable particle sizing in the range of 19 $\mathrm{nm}$ to $107 \mathrm{~nm}$ using less than $150 \mu \mathrm{L}$ of solution (Figure 1). Rapid measurements using these devices surveyed a range of solution composition and temperature to determine surfactant dissolution behavior. Simple extension of these measurements will enable phase mapping of nanostructured solutions. The instruments were designed to be integrated with existing NIST techniques for synthesis, spectroscopy, and image
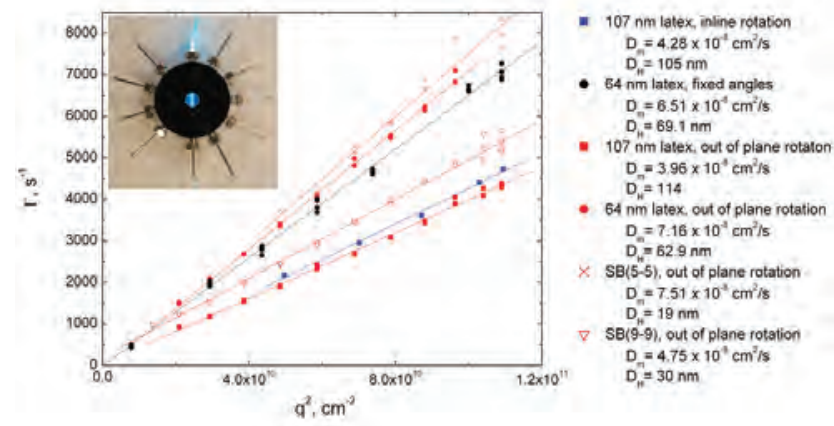
$D_{n}^{\prime \prime}=30 \mathrm{~nm}$
$D_{\mu}=30 \times 10$

Figure 1: Diffusion measurements and particle sizing of latex spheres and block copolymer micelles conducted on a microfluidic device. Inset: Photograph of a fixed, multi-angle device.

analysis of polymer solutions in microfluidic devices. the developing industry.

Progress was also made on additional methods, including the validation and integration of droplet library fabrication, photopolymerization, and shrinkage measurements for methacrylate-based resins [The Analyst 131, 1027

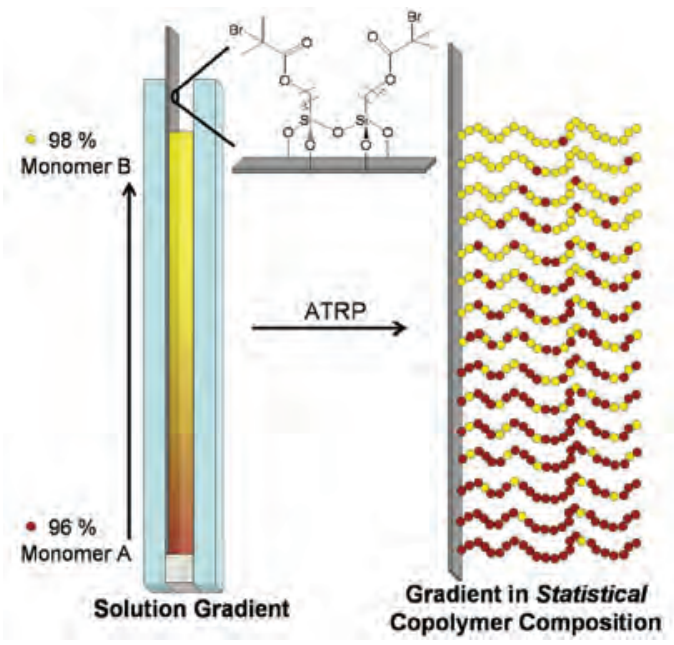

Figure 2: : Illustration of a solution gradient stabilized in a confined microchannel (left) that was used to construct a polymer brush surface with a gradient in statistical copolymer composition (right).

(2006).]. Correlations between monomer composition, extent of conversion, and shrinkage were matched with off-chip measurements.

Microfluidic confinement has also enabled the stabilization of solution gradients, as validated by fiber-optic Raman spectroscopy. The power of such a solution gradient was demonstrated by fabrication of a statistical copolymer brush surface containing a gradient in composition [Fig. 2; Adv. Mat. 18, 1427 (2006)], a gradient heretofore unobtainable, and thus valuable in characterizing thin film and nanoparticle behavior.

Visiting scientists from CSIRO and BASF performed research in the NIST laboratories this year. Microfluidic device design, measurement strategies for polymerization, and use of microscopy and spectroscopy tools were transferred to both organizations. A new joint project on the library design and characterization of surfactant solutions will eventually be conducted at CSIRO using NIST technology.

\section{Contributors and Collaborators}

T.Q. Chastek, C. Xu, S.E. Barnes, K.A. Page, M.J. Fasolka, E.J. Amis (Polymers Division, NIST); S. Müssig (BASF); C. Fong (CSIRO) 


\section{Quantitative Polymer Mass Spectrometry}

Matrix-assisted laser desorption ionization time-offlight mass spectrometry (MALDI-TOF-MS) has been demonstrated as a method for absolute molecular mass distribution measurement of synthetic polymers via the creation of Standard Reference Material ${ }^{\circledR}$ 2881. This means determining a comprehensive uncertainty budget for a complex measurement technique that must include both Type A and Type B uncertainties.

\section{William E. Wallace}

C urrently, all absolute molecular mass polymer standards provide a single moment (e.g., $\mathrm{M}_{\mathrm{n}}$ or $\mathrm{M}_{\mathrm{w}}$ ) of the molecular mass distribution (MMD). (By absolute it is meant that the standard is based on direct measurement of a physical property, and not on calibration against another polymer.) We have created a comprehensive strategy to create an absolute molecular mass distribution polymer Standard Reference Material ${ }^{\circledR}$ using matrix-assisted laser desorption/ionization time-of-flight (MALDI-TOF) mass spectrometry. To create such a standard, our methods must be demonstrably free of operator bias (intentional or unintentional). Furthermore, the Type A ("random") and Type B ("systematic") uncertainties must be determined for each part of the experiment. The required unbiased, repeatable steps are: 1 ) sample preparation, 2) instrument tuning, 3) data collection, and 4) data analysis. These steps ultimately lead to a signal intensity calibration function (and, most importantly, its associated uncertainty) that relates the true MMD of the analyte to the MMD measured by mass spectrometry.

We used a narrow polydispersity polystyrene initiated with an n-octyl group, and having a peak mass $\left(\mathrm{M}_{\mathrm{p}}\right)$ of $9000 \mathrm{u}$. To this polymer we gravimetrically mix two n-butyl-initiated polystyrenes, one having an $\mathrm{M}_{\mathrm{p}}$ of $6000 \mathrm{u}$ and the other an $\mathrm{M}_{\mathrm{p}}$ of $12000 \mathrm{u}$. The different end groups allow us to distinguish between different peaks in the mass spectrum, as shown in the top panel of the figure (right). By controlling the gravimetric mix, we created a signal-intensity calibration curve using a Taylor's expansion around a central mass peak as our mathematical model. We used electrospray methods to create uniform MALDI targets that are free of "sweet spots" that could be reproducibly made, and whose preparation could be described precisely. Removal of the gross uncertainties inherent in typical MALDI sample preparation methods (e.g. "hand spotting") is critical for quantitation work. With gradient-free numerical optimization methods (see Technical Highlight in this Report) we located the instrument parameter settings in 5-dimensional space (extraction, lens, and detector voltages; laser intensity; and extraction delay time) that give the least bias in the molecular mass distribution, as well as the Type B uncertainties for the measurement. We do this by comparing the difference between the gravimetric mixture ratios with the ratios found by mass spectrometry until an optimal set of instrument settings is found. Data collection is randomized to prevent unknown changes in experiment procedure or instrument performance from creating a systematic bias in the data over periods of hours, days, and weeks. Using our time-series segmentation method, MassSpectator (see W.E. Wallace, A.J. Kearsley, and C.M. Guttman, “An Operator Independent Approach to Mass Spectral Peak Identification and Integration," Analytical Chemistry 76, 2446 (2004) and A.J. Kearsley, W.E. Wallace, and C.M. Guttman, "A Numerical Method for Mass Spectral Data Analysis," Applied Mathematics Letters 18, 1412 (2005), we automatically identify and integrate mass peaks to give a MMD, as shown in the bottom panel of the figure. Finally, using standard additions of the three polystyrenes (and two other polystyrenes with intermediate average masses), we created a signal intensity calibration curve as a function of molecular mass across the distribution. Variations in relative ratios of the three polymers let us determine the Type A uncertainty in the calibration curve.

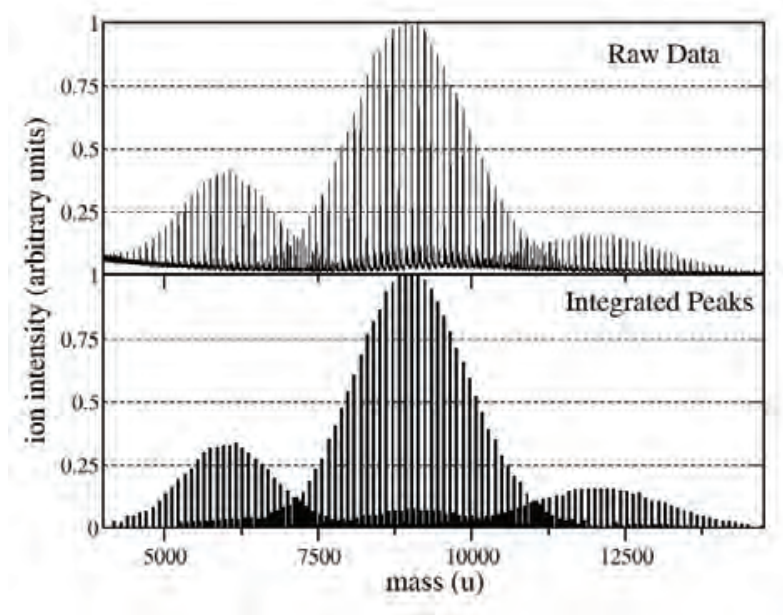

\section{Contributors and Collaborators}

M.Y.M. Chiang, S. Guo, J.H. Kim, E.A. Wilder, W. Zhang (Polymers Division, NIST); Daisuke Kawaguchi (Nagoya University); Gareth Royston (University of Sheffield) 


\section{Biomaterials}

Rapid development of medical technologies depends on the availability of adequate methods to characterize, standardize, control, and mass produce them. To realize this goal, a measurement infrastructure is needed to bridge the gap between the exponentially increasing basic biomedical knowledge and clinical applications. The NIST Materials Science and Engineering Laboratory (MSEL) Biomaterials Program is a collaborative effort creating a new generation of performance standards and predictive tools targeting the metrology chain for biomedical research.

Today, all areas of materials science confront real systems and processes. In the biomaterials arena, we can no longer advance science by simply studying ideal model systems. We must comprehend complex realistic systems in terms of their structure, function, and dynamics over the size range from nanometers to millimeters. MSEL is uniquely positioned to make a major contribution to the development of measurement infrastructure through three focus areas: Systems Biology, Bioimaging, and Nanobiosensing.
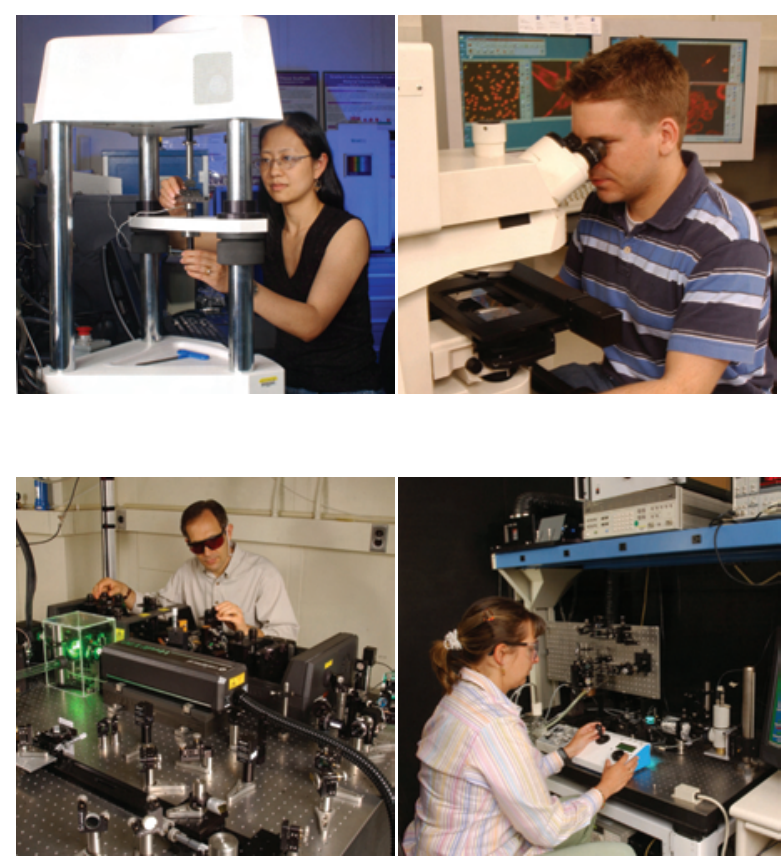

Contact: Marcus Cicerone 


\section{D Tissue Scaffolds}

We are developing reference materials and noninvasive analytical methods to meet measurement needs related to $3 D$ tissue engineering (TE). These methods and reference materials will replace destructive end-point methods currently in use, and in so doing, will facilitate more efficient and effective TE scaffold testing and design.

\section{Marcus T. Cicerone}

$\mathrm{R}$ esult-focused rational design of 3D TE scaffolds, one Holy Grail of tissue engineering, requires first that cell response can be monitored to known conditions within the bulk of the scaffold. We are developing noninvasive characterization methods that will replace some of the destructive end-point methods currently in use. The methods we are developing will result in quantifiable, reliable metrics that are relevant to success of TE constructs in properly supporting cell and tissue growth. Cells in 3D constructs respond to perfusion conditions, local surface morphology, and surface chemistry, as well as a number of other mechanical, chemical, and electrical stimuli. Work under this project focuses on characterizing the first three of these factors, as well as cell response to them.

\section{Reference Scaffold and Structural Analysis -} There is a continuing need for reference materials and standard artifacts in this field. We are in the final stage of generating a 3D reference scaffold for porosity. These artifacts will be used as standards against which to evaluate scaffold porosity and permeability; the primary characterization data are permeability and porosity. Analysis of 3D images obtained by micro-computed tomography $(\mu \mathrm{CT})$ plays a major role in scaffold characterization. We expect to complete an interlaboratory comparison for determining confidence limits in specifications by December 2006. The work is a collaborative project through ASTM (Task Force F04.42.06). This work has also spurred generation of an ASTM draft guide for scaffold characterization (in preparation).

In-situ Cell and Tissue Imaging - Optical and other imaging modes have revolutionized research into many areas, including cell-material interactions. Optical imaging in 3D TE constructs poses two important problems: the rapidly dividing cells are sensitive to high light flux, and 3D scaffolds generally provide poor platforms for high-resolution imaging due to opacity. In addressing the former, we continue to develop broadband coherent anti-Stokes Raman scattering microscopy
( $\mu$-CARS) as a powerful, noninvasive method for determining cell health and development within tissue scaffolds. Progress this year has included a new method to eliminate nonresonant background so that weak spectral features in the fingerprint region can be detected [Kee et al., Optics Express 14, 3631 (2006)].

We have demonstrated a solution to another major hurdle for imaging in 3D scaffolds by developing an optically clear tissue scaffold. This scaffold is fabricated by salt-leaching from a photopolymerized glassy polymer that has been used in dental applications. Characterization of these scaffolds with respect to morphology and mechanical properties has been submitted to Biomaterials (S. Lin-Gibson, et al.). A report on behavior of osteoblast cells on these scaffolds has been published [F.A. Landis et al., Biomacromolecules 7, 1751 (2006)].
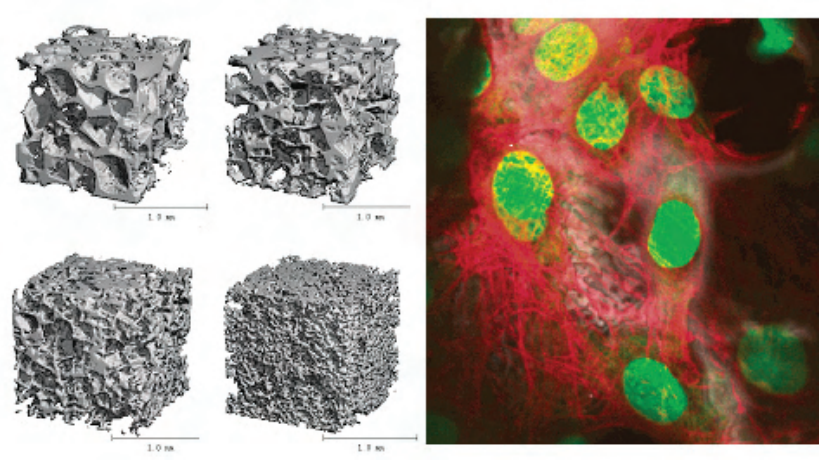

Figure 1: Optically clear salt-leached scaffolds made with varying porosity and pore size allows large range in mechanical properties as well as clear imaging of cells as far as $400 \mu \mathrm{m}$ into scaffold.

Applicability of 2D Results to 3D Scaffolds Surfaces of many 3D scaffold types present to cells as having varying degrees of curvature, however analysis of cell response to surface chemistry is most conveniently performed on flat surfaces. We have shown that in some cases the results obtained in 2D are retained in 3D scaffolds. Distinct compositions of phase-separated blends of tyrosine-derived polycarbonates have been shown to elicit marked differences in proliferation and differentiation response in 2D. We have recently demonstrated that these results carry over to 3D.

\section{Contributors and Collaborators}

Y.M. Chiang, M.T. Cicerone, J. Cooper, J.P. Dunkers, S. Lin-Gibson, F. Phelan, J.R. Smith, T. Dutta Roy, F.Wang, T.W. Kee (Polymers Division, NIST); J. Kohn (Rutgers) 


\section{Combinatorial Methods for Tissue Engineering}

The goal of this project is to develop measurement methods and associated materials to characterize the interactions, at the surface, between cells and biologically active materials for use in optimizing tissue-engineered products.

\section{Matthew L. Becker}

$T_{t}$ he diversity of functional species, distinct structural features, and multiple hierarchies within classes of functional biomaterials create significant challenges to characterization. Solutions to these challenges require novel approaches to measurements, including the development of new methods and instrumentation. Our program seeks to develop combinatorial tools and methods to identify the critical physico-chemical properties influencing the biological performance of biomaterials.

Morphology Gradient Libraries - We are fabricating single and multi-variable continuous gradient libraries to generate linear variations in important physical properties, including roughness, surface energy, crystallinity, and phase separation. This approach affords opportunities to quickly identify compositions and physical properties exhibiting favorable cell-material interactions using automated characterization and data acquisition methods, and narrow the physical parameter space where higher levels of characterization are required.
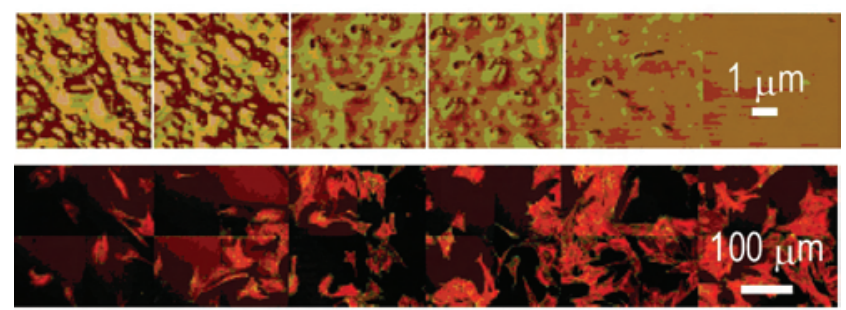

Figure 1: Diffraction patterns collected over a range of sample rotation angles. The distance between the pronounced horizontal ridges provides the sidewall angles $\beta$, while the relative intensity and placement

One of our focus areas is to develop physical and spectroscopic methods to measure the extent and associated physico-chemical changes resulting from phase separation. Phase separation is a critical component in solution processing, materials optimization, and medical device manufacturing. The various changes in properties have been shown to influence preferential protein adsorption profiles that influence the biological responses.
Bioactive Ligand Gradients - We are designing bioactive ligand density gradients to identify optimum lignad densities for directed cell adhesion, proliferation, and differentiation. We are combining traditional and automated bio-imaging techniques with emerging microanalytical techniques to obtain data that links substrate properties with morphology and biochemical signatures of protein and RNA expression in small cell populations.

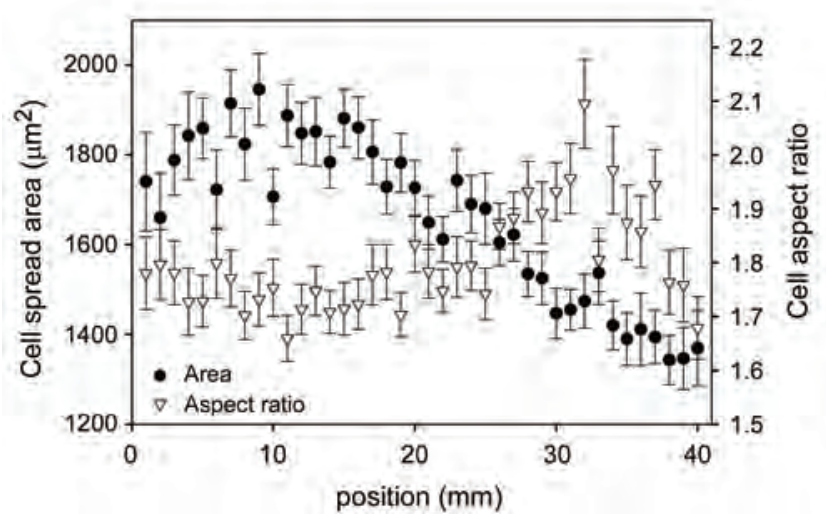

Figure 2: The plot above depicts cell area and aspect ratio as a function of position on a peptide functionalized self assembled monolayer possessing a gradient in RGD density, a peptide commonly used in cell adhesion applications.

Cell morphological, viability, and biomarker measurements are being measured on similar 2D and 3D substrates and their results compared among them and with existing data of in vivo samples. These welldefined substrates are being used to push the sensitivity limits of current methods and if successful, the correlations will increase the ability of in vitro cell-based measurements to predict cellular behavior in vivo.

We anticipate these efforts will provide the foundation for a framework, which would guide the early assessments of in vitro and in vivo material performance, and establish a series of metrologies for biocompatibility thresholds. These measurement solutions would provide useful guidance to materials development efforts, manufacturing process optimization, and regulatory agencies.

\section{Contributors and Collaborators}

C.G. Simon, N.D. Gallant, M.J. Kipper, K.E. Roskov, M.D. Roy, M.T. Cicerone (Polymers Division, NIST); J. Kohn, P. Holmes (New Jersey Center for Biomaterials, Rutgers University); D.L. Kaplan (Tissue Engineering Resource Center, Tufts University) 


\section{Dental Polymers and Composites: Developing a Test Suite for Total Materials Characterization}

The objective of this project is to develop combinatorial platforms for characterizing salient material properties of candidate dental polymers. These platforms will aid others in the development and optimization of polymers for restorative composites with improved performance.

\section{Sheng Lin-Gibson and Nancy J. Lin}

$\mathrm{P}_{\mathrm{r}}^{\mathrm{o}}$ lymeric dental composites are widely used in the restoration of anterior lesions and small to medium sized defects in the posterior region of the mouth. While significant improvements have been made in the composites' properties, their long term performance is still less than optimal. A significant effort has been devoted to improve the composite properties by designing new dental polymers. For the matrix polymer alone, a large number of material and processing parameters influence the material properties and biological responses. High-throughput and combinatorial methods are ideal for the purpose of characterizing these materials because of their faster data acquisition and ability to span a wide parameter space, and equal processing conditions for a given specimen, resulting in a lower experimental error rate.

\section{2,2-bis[4-(2-hydroxy-3-methacryloxypropoxy)} phenyl] propane (BisGMA) and triethylene glycol dimethacrylate (TEGDMA) are two commercially important dental monomers. 2D gradient samples of a model dental polymer consisting of a dimethacrylate mixture (BisGMA and TEGDMA) varying in composition and conversion were fabricated (Figure 1). A broad conversion range was obtained for all compositions. The conversions were measured using near infrared (IR) spectroscopy, and the mechanical properties (elastic modulus and hardness) were determined using nanoindentation with the continuous stiffness method. Our previous work demonstrated a strong correlation between vinyl conversion and mechanical properties of the cross-linked polymer.

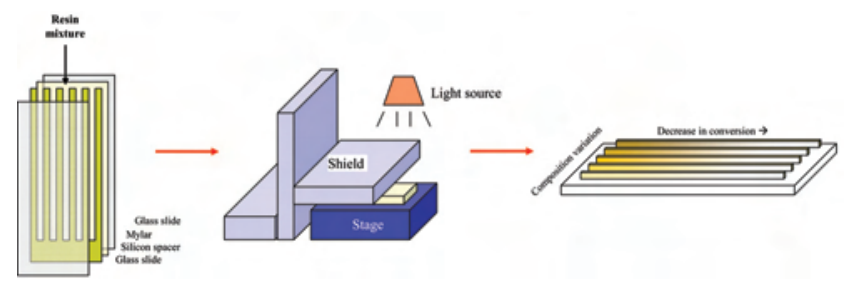

Figure 1: Fabrication of 2D gradient samples.
Cell viability on the 2D gradient samples was assessed using fluorescence microscopy (Figure 2). As the degree of conversion increased, cell attachment and viability increased. Compositions with increasing TEGDMA content had increased cell viability, but the effects were subtler.

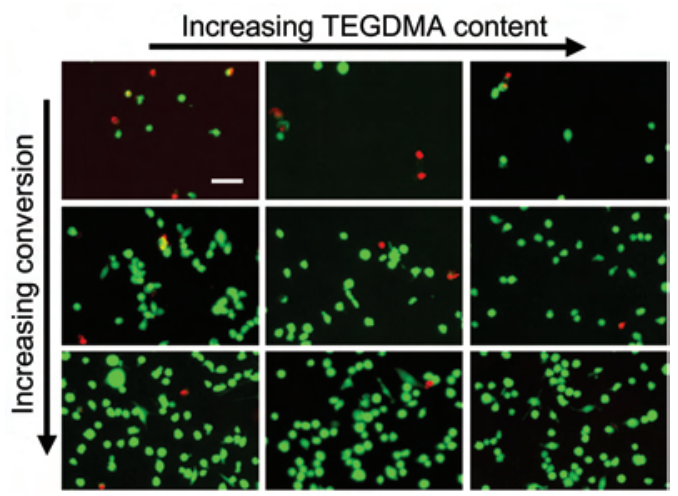

Figure 2: Fluorescent microscopy showing viability of cells cultured on 2D gradient samples. Live cells are stained green, and dead cells are stained red. Scale bar $=50 \mu \mathrm{m}$.

Two indicators for material biocompatibility are cell viability and cell attachment density, both of which can be quantified (Figure 3 ). In general, cell viability remained high until the conversion fell below $44 \%$. A high degree of cell attachment required a higher conversion. Similar trends were seen for all compositions.
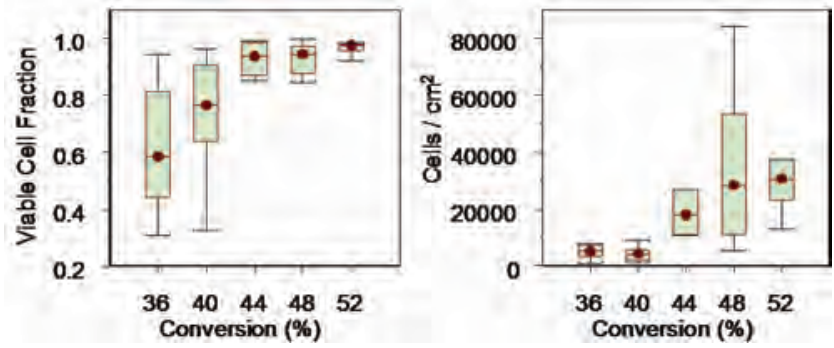

Figure 3: Cell viability (left) and cell density (right) measured as a function of methacrylate conversion for a single composition.

Continuous variable gradients are efficient for screening biological effects arising from the composition and degree of conversion. Other potential combinatorial/ high-throughput techniques are also being evaluated for their ability to screen structure-properties relationships in dental materials, including relating conversion to polymerization shrinkage.

\section{Contributors and Collaborators}

\section{J.M. Antonucci (Polymers Division, NIST)}




\section{Metrology for Biomechanical Effects on Cell/Material Interactions}

\begin{abstract}
Many tissue engineered medical products are cultured under mechanical stimuli to mimic the physiological environment. This approach is currently addressed in an ad hoc way to demonstrate how cells respond under macroscopic stress conditions. However, stress at the cellular level is not well characterized. This project will develop a measurement infrastructure consisting of instrumental techniques, mathematical modeling, and characterization protocols to determine local stresses on cells from macroscopic stresses and to quantify the effect of these stresses on cell morphology, phenotype, and biochemical signaling.
\end{abstract}

\section{Joy P. Dunkers}

$\mathbf{M}$ etrology Development - Mechanotransduction is the process by which cells convert mechanical stimuli into biochemical signals. Most cell functions are influenced by mechanical stimuli: proliferation, migration, differentiation, apoptosis, and protein synthesis. Much work has been done to identify the specific molecular signals activated for various phenotypes, and to correlate the macroscopic stress/strain on the flexible substrate to cell response. However, there are currently no standard protocols on how these studies are conducted, or how the real time stress/strain local to the cell is determined. We believe that the existence of many apparently contradictory results in the literature is related to this lack of consistent analysis. We are creating a measurement platform to quantify the actual stress transferred to the cell and to correlate it to cellular response in 2-D and 3-D cultures. This platform will be useful to researchers, as well as to regulatory agencies, as they seek new methods to test tissue engineered medical products.

In this investigation, smooth muscle cells were selected as the model system to examine the transition from synthetic to contractile phenotypes under biaxial stress. A combination of culture techniques, bioreactor designs, and cell-based assays were used to image, quantify, and predict biological responses as a function of mechanical stimuli. Studies include dynamic culture testing, advances in optical and fluorescence in vitro imaging, genetic profiling, and mathematical/computational modeling. This research platform expands across the tissue engineering spectrum, starting from the simplest case of non-confluent cells to proliferation and organization into tissues.
This year we developed methods to robustly attach and thoroughly characterize extracellular matrix (ECM) proteins on the deforming substrate. Here, the ECM protein is fibronectin (FN) grafted to the deforming polydimethylsiloxane via a silane coupling agent. We characterized the FN coating in terms of surface roughness, uniformity, thickness, conformation, and rigidity. Response of smooth muscle cells (shown below, stained for focal adhesions and actin cytoskeleton) to the grafted FN was characterized by proliferation and live/dead assays. These baseline results will serve to evaluate the durability of the attached FN after the biaxial stretching.

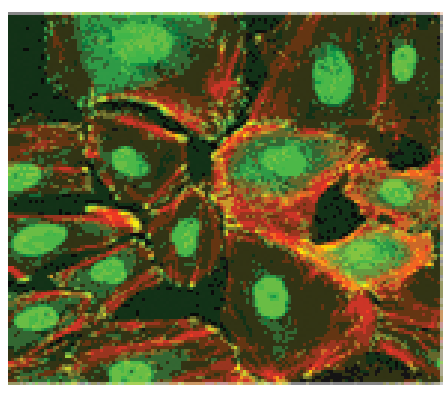

Mathematical Modeling - Previous in vitro studies using cells cultured on substrates show that 1 ) cells sense the variation in substrate rigidity by modifying their morphology; 2) cells subjected to uniaxial stretch elongate perpendicular to the stretch direction; and 3) cells show preference to specific substrate stiffness on a stiffness gradient (durotaxis).

Guided by this, we developed a mathematical model of cell-substrate adhesion to explain the dependency of cell morphology and migration on substrate mechanics. The hypothesis is that the morphology of a cell adhering to a substrate is characterized by the competition between cell and substrate strain energies and interfacial energy (work of adhesion) at the cell periphery. The final configuration of the cell morphology is determined by the total free energy minimum of the cell/substrate system. This work is discussed further in the Highlights section of this report. [Yong Ni and Martin Y.M. Chiang, "Cell Morphology and Migration Linked to Substrate Rigidity,” Soft Matter 3, 1285 (2007).]

\section{Contributors and Collaborators}

L. Pakstis, M.Y.M. Chiang, Y. Ni, N. Lin, L. Henderson, J. Cooper, M. Cicerone (Polymers Division, NIST); T. Quinn, T. Oreskovic (Materials Reliability Division, NIST); T. Vorburger, A. Zheng (Precision Engineering Division, NIST); J. Taboas, R. Tuan (NIAMS, NIH) 



\section{Materials for Electronics}

The U.S. electronics industry faces strong international competition in the manufacture of smaller, faster, more functional, and more reliable products. Many critical challenges facing the industry require the continual development of advanced materials and processes. The NIST Materials Science and Engineering Laboratory (MSEL) works closely with U.S. industry, covering a broad spectrum of sectors including semiconductor manufacturing, device components, packaging, data storage, and assembly, as well as complementary and emerging areas such as optoelectronics and organic electronics. MSEL has a multidivisional approach, committed to addressing the most critical materials measurement and standards issues for electronic materials.

Our projects are conducted in collaboration with partners from industrial consortia (e.g., SEMATECH), individual companies, academia, and other government agencies. The program is strongly coupled with other microelectronics programs within the government such as the National Semiconductor Metrology Program (NSMP). Materials metrology needs are also identified through the International Technology Roadmap for Semiconductors (ITRS), the International Packaging Consortium (IPC) Roadmap, the IPC Lead-free Solder Roadmap, the National Electronics Manufacturing Initiative (NEMI) Roadmap, the Optoelectronics Industry Development Association (OIDA) Roadmap, and the National Magnetic Data Storage Industry Consortium (NSIC) Roadmap.

Contact: Christopher L. Soles

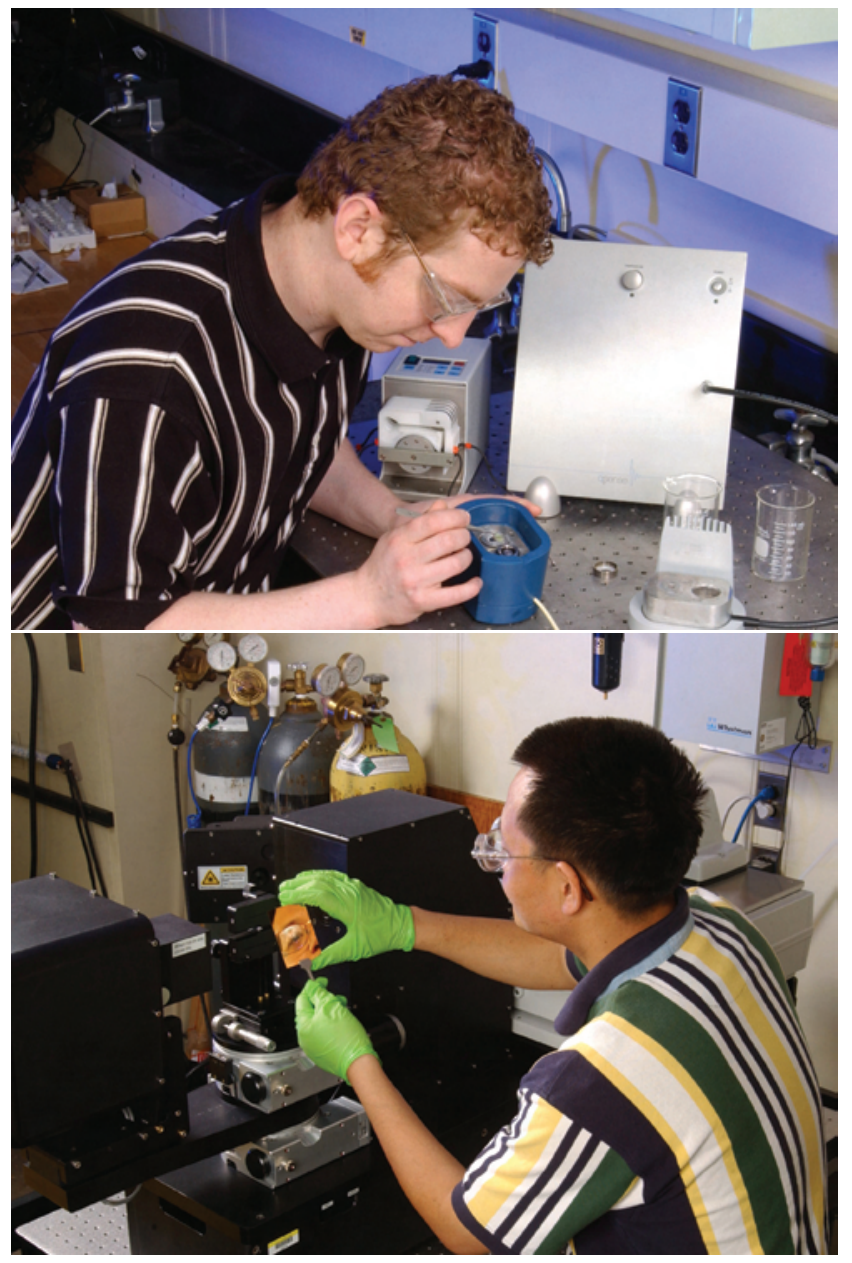




\section{Dimensional Metrology with Small Angle X-Ray Scattering}

The feature size in microelectronic circuitry is ever decreasing, and now approaches the scale of nanometers. This trend creates a need for new measurements capable of non-destructive measurements of small features consisting of a variety of materials (polymers, nanoporous thin films, metals), with sub-nanometer precision. In this project, we are developing small angle $x$-ray scattering for the non-destructive measurements of the pitch, linewidth, sidewall angle, average cross section, and line edge roughness in nanoscale patterns. Other applications of this technique in areas such as nano-rheology and nanofabrication are being explored.

\section{Wen-li Wu and Ronald L. Jones}

$\mathrm{T}^{\mathrm{k}}$ he demand for increasing computer speed and deceasing power consumption continues to shrink the dimensions of individual circuitry components toward the scale of nanometers. When the smallest, or "critical”, dimensions are $<40 \mathrm{~nm}$, the acceptable tolerance will be $<1 \mathrm{~nm}$. This requirement creates significant challenges for measurements based on electron microscopy and optical scatterometry. In addition, the continuing development of new materials for extreme ultraviolet photoresists, nanoporous low-k dielectrics, and metallic interconnects all require high precision dimensional measurements for process development and optimization.

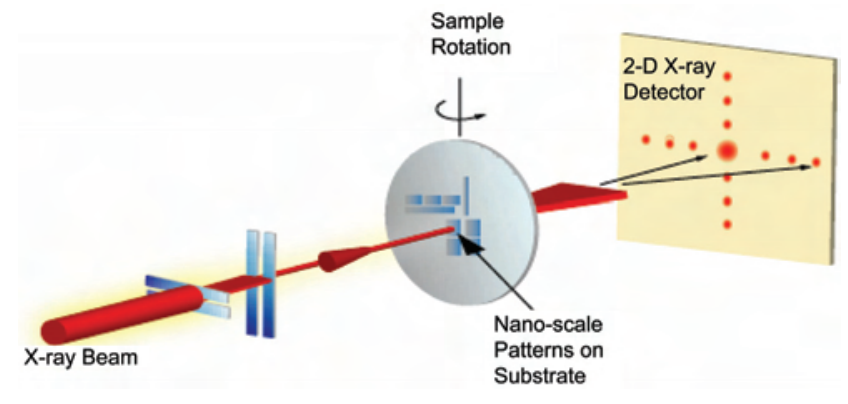

Figure 1: Schematic of the CD-SAXS geometry, showing the $x$-ray beam (solid line) in transmission through the patterned sample.

To address this need, we are developing a high precision X-ray based metrology, Critical Dimension Small Angle X-ray Scattering (CD-SAXS). This technique is capable of non-destructive measurements of test patterns routinely used by microelectronic industries to monitor their fabrication process. A collimated monochromatic $\mathrm{x}$-ray beam of sub-Ångström wavelength is used to measure the pattern dimensions on a substrate in transmis- sion mode. CD-SAXS has previously demonstrated a capability for sub-nm precision for periodicity, line width, sidewall angle, and height measurements.

This year, we have extended the capabilities to better quantify roughness. Structures were designed with line edges close to a sine wave with a nominal wavelength of $150 \mathrm{~nm}$ and amplitudes ranging from $20 \mathrm{~nm}$ to $60 \mathrm{~nm}$. Three classes of structures were measured: (1) centerline roughness (CLR), where the center of mass of the line varies sinusoidally but the line width remains constant; (2) line width roughness (LWR), where the centerline follows a straight line and the line width varies as two opposing sine waves; and (3) line edge roughness (LER), where the line edges have some randomness in its phase angle relative to its neighbor. These controlled structures produced observable "satellite" diffraction peaks that run parallel to the main diffraction axis. The peak positions provide the wavelength of the roughness, while their intensity revealed the amplitude of the periodic component to the roughness.
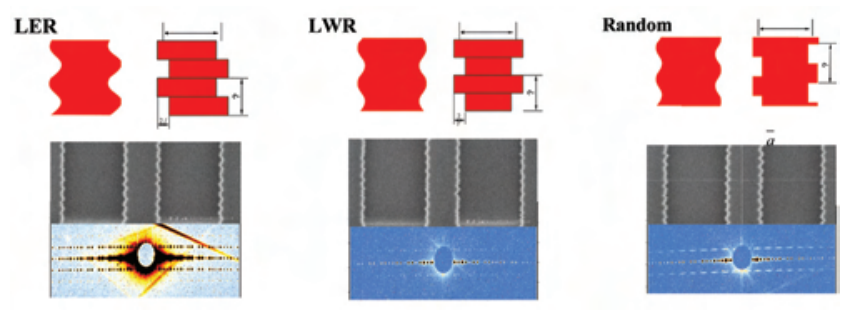

Figure 2: Schematic of model line edge roughness structures with the top-down SEM micrograph and the resulting SAXS data.

Also this year, NIST completed installation of the world's first laboratory based CD-SAXS instrument. This instrument is incorporates a rotating anode source with two different targets, advanced focusing optics, and a Bonse-Hart camera. The result is a flexible system that can address both the development of CD-SAXS for the semiconductor industry and advanced structural measurements of nanostructured materials in solution or in the bulk.

\section{Contributors and Collaborators}

C. Wang, D. Ho, H. Lee, C. Soles, H. Ro, V. Tirumala, E. Lin (Polymers Division, NIST); K. Choi, B. Rice, G. Thompson (Intel); B. Bunday (ISMI); S. Knight (Office of Microelectronics Programs, NIST); J. Villarrubia, A. Vladar (MEL, NIST); P. Butler (Center for Neutron Research, NIST); Y. Liu (SEMATECH); Q. Lin, A. Grill, H. Kim (IBM); K. Char, D. Yoon (Seoul National University); J. Watkins (U. Mass-Amherst) 


\section{Organic Electronics}

Organic electronics has dramatically emerged in recent years as an increasingly important technology encompassing a wide array of devices and applications, including embedded passive devices, flexible displays, and photovoltaic cells. Device performance, stability, and function critically depend upon charge transport and material interaction at the interfaces of disparate materials. We develop measurement methods to characterize the electronic and interfacial structure of organic electronics materials with respect to processing methods, processing variables, and materials characteristics.

\section{Dean M. DeLongchamp}

$\mathrm{O}$ rganic electronic devices are projected to revolutionize new types of integrated circuits through new applications that take advantage of low-cost, high volume manufacturing, nontraditional substrates, and designed functionality. The current state of organic electronics is slowed by the concurrent development of multiple material platforms and processes, and a lack of measurement standardization between laboratories. A critical need exists for new diagnostic probes, tools, and methods to address these technological challenges.
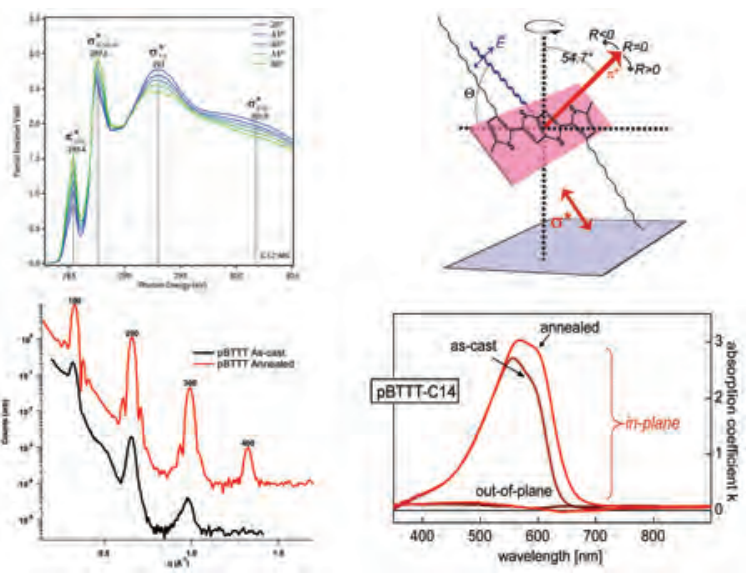

Figure 1: Several different experimental methods (NEXAFS, $X R D$, and ellipsometry) are brought together to determine the structure of $20 \mathrm{~nm}$ films to determine correlations with device performance.

Organic electronics presents different measurement challenges from those identified for inorganic devices. We are developing an integrated suite of metrologies to correlate device performance with the structure, properties, and chemistry of materials and interfaces. We apply new measurement methods to provide the data and insight needed for the rational and directed development of emerging materials and processes. Studies include electrical measurements of organic semiconductor films, the effect of branched end-group substitutions on molecular packing, growth mechanisms of evaporated thin films, and the development of combinatorial methods to characterize materials rapidly.

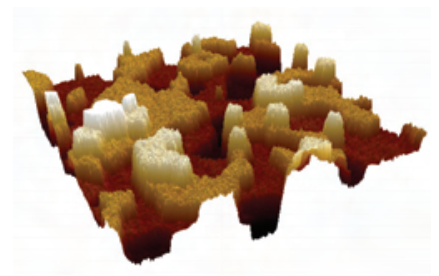

Figure 2: AFM image from terraced structure of a high carrier mobility polythiophene thin film.

This year, significant progress was made to identify the molecular characteristics of newly developed, high carrier mobility poly(2,5-bis(3-alkylthiophen-2-yl) thieno[3,2-b]thiophenes). These polymers exhibit the highest mobility for a polymer material due to exceptional order throughout the film. Near edge x-ray absorption fine structure (NEXAFS), atomic force microscopy (AFM), ellipsometry, Fourier transform infrared (FTIR) spectroscopy, and x-ray diffraction (XRD) measurements were able to highlight the influence of substrate surface on the film morphology, to determine the tilt angle of the conjugated planes, and to highlight the importance of the side-chain packing density on the structure formation.

Several activities highlighted the increasing need for advanced measurements and materials science in organic electronics. A U.S. Measurement System Workshop was held on "Large-area Flexible Electronics and Photonics” (http://usms.nist.gov/workshops/ macroelectronics.htm) to determine measurement needs to enable this emerging technology area. Also, NIST staff co-chaired and contributed to the development of a technology roadmap for printed and organic electronics with iNEMI.

\section{Contributors and Collaborators}

R.J. Kline, J. Obrzut, Y. Jung, T. Psurek, B. Vogel, M. Fasolka, C. Chiang, E. Lin (Polymers Division, NIST); D. Fischer (Ceramics Division, NIST); M. Gurau, L. Richter (CSTL, NIST); D. Gundlach, C. Richter, O. Kirillov, (EEEL, NIST); I. McCulloch, M. Heeney (Merck Chemicals), L. Lucas, G. Jabbour (Arizona State University); M. Chabinyc, J. Northrup (PARC); A. Murphy, J.M.J. Frechet, P. Chang, V. Subramanian (U. C. Berkeley); M. Ling, Z. Bao (Stanford University); Y. Wu, B. Ong (Xerox); M. Toney (Stanford Synchrotron Radiation Laboratory) 


\section{Polymer Photoresists for Next-Generation Nanolithography}

Photolithography, the process used to fabricate integrated circuits, is the key enabler and driver for the microelectronics industry. As lithographic feature sizes decrease to the sub-32 $\mathrm{nm}$ length scale, challenges arise because photoresists appear to be reaching their resolution limits. Unique high-spatial resolution measurements are developed to reveal limits on materials and processes that challenge the development of photoresists for next-generation sub-32 nm lithography.

\section{Vivek M. Prabhu}

$\mathrm{P}_{\mathrm{t}}^{\mathrm{h}}$ hotolithography is the driving technology used by the microelectronics industry to fabricate integrated circuits with ever decreasing sizes. This fabrication technology is also rapidly being adopted in emerging areas in optoelectronics and biotechnology, requiring the rapid creation of nanoscale structures. In this process, a designed pattern is transferred to the silicon substrate by altering the solubility of areas of a polymer-based photoresist thin film through an acid catalyzed deprotection reaction after exposure to radiation through a mask. To fabricate smaller features, next generation photolithography will be processed with shorter wave lengths of light requiring photoresist films less than $100 \mathrm{~nm}$ thick and dimensional control to within $2 \mathrm{~nm}$.

We work closely with industrial collaborators to develop high-spatial resolution and chemically specific measurements to understand changes in material properties, interfacial behavior, and process kinetics at nanometer scales that can significantly affect the patterning process.

This year, we worked with SEMATECH to determine materials sources of line-edge roughness in 193-nm photoresists, and with Intel Corporation to investigate the effect of extreme-ultraviolet (EUV) exposure on pattern resolution of model EUV photoresist materials. We continue to provide new insight and detail into the complex physico-chemical processes used in advanced chemically amplified photoresists. Measurement methods include $\mathrm{x}$-ray and neutron reflectivity (XR, NR), small angle neutron scattering (SANS), near - edge $\mathrm{x}$-ray absorption fine structure (NEXAFS) spectroscopy, solid state nuclear magnetic resonance (NMR), quartz crystal microbalance (QCM), infrared variable-angle spectroscopic ellipsometry; fluorescence correlation spectroscopy (FCS), and atomic force microscopy.
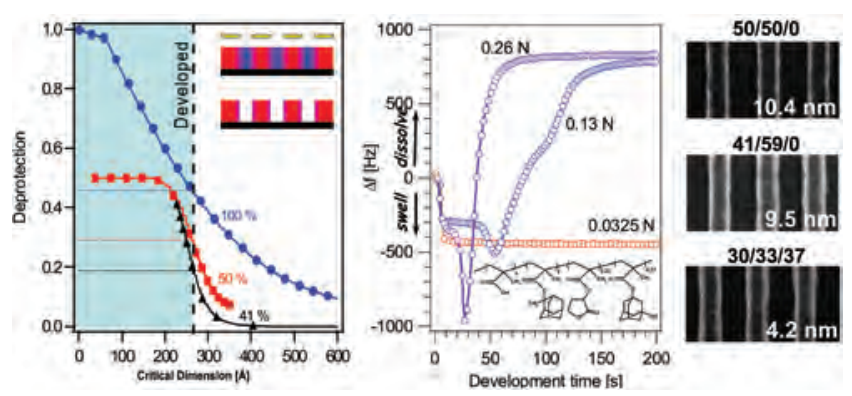

Figure 1: Copolymer composition effect on deprotection front measured by NR. Swelling and dissolution behavior of polymer thin films controlled by developer normality quantified by QCM. Evaluation of three model copolymer resists for LER for $100 \mathrm{~nm}$ line/space imaged with $193 \mathrm{~nm}$ photolithography.

Photoresists are multi-component mixtures that require dispersion of additives, controlled transport properties during the interface formation, and controlled dissolution behavior. The fidelity of pattern formation relies on the materials characteristics. Hence, we examine the influence of copolymer compositions, molar mass, and photoacid generator additive size to determine the root causes of image quality by highlighting the polymer physics and chemistry. In addition, our collaborators test our hypothesis using $193 \mathrm{~nm}$ and EUV lithographic tests.

Accomplishments for this past year include quantification of the copolymer composition and quencher additives effect on photoacid-reaction diffusion deprotection front for resolution and roughness fundamentals by NR, advancement of photoresist / liquid interfaces for dissolution fundamentals by QCM, effect of exposure dose on the reaction-front in model EUV materials, photoacid generator miscibility and dispersion in complex photoresist co- and ter-polymers by NMR, aqueous immersion dependence on photoacid generator leeching and depth profile by NEXAFS, and development of cross-correlation FCS with nanopositioning for labeled-polymer diffusivity in bulk and at interfaces.

\section{Contributors and Collaborators}

B. Vogt, A. Rao, S. Kang, D. VanderHart, K. Lavery, W. Wu, E. Lin (Polymers Division, NIST); D. Fischer, S. Sambasivan (Ceramics Division, NIST); S. Satija (NIST Center for Neutron Research, NIST); K. Turnquest (SEMATECH); Kwang-Woo Choi (Intel); R. Allen, W. Hinsberg, H. Ito (IBM Almaden Research Center); R. Dammel, F. Houlihan (AZ Electronics); J. Sounik, M. Sheehan (DuPont Electronic Polymers) 


\section{Nanometrology}

Nanotechnology will revolutionize and possibly revitalize many industries, leading to new and improved products based on materials having at least one dimension less than $100 \mathrm{~nm}$. The federal government's role in realizing the full potential of nanotechnology is coordinated through the National Nanotechnology Initiative (NNI), a multi-agency, multi-disciplinary program that supports research and development, invests in a balanced infrastructure, and promotes education, knowledge diffusion, and commercialization in all aspects of nanoscale science, engineering, and technology. NIST's unique and critical contribution to the NNI is nanometrology, defined as the science of measurement and/or a system of measures for nanoscale structures and systems. NIST nanometrology efforts focus on developing the measurement infrastructure - measurements, data, and standards - essential to advancing nanotechnology commercialization. This work provides the requisite metrology tools and techniques and transfers enabling measurement capabilities to the appropriate communities.

The NIST Materials Science and Engineering Laboratory (MSEL) plays a vital role in nanometrology work at NIST with efforts in four of the seven NNI Program Component Areas - Instrumentation Research, Metrology and Standards for Nanotechnology; Nanomaterials; Nanomanufacturing; and Fundamental Nanoscale Phenomena and Processes. Innovative projects across MSEL are defining and addressing the forefront research issues in these areas.

Contacts: Alamgir Karim; Kalman Migler

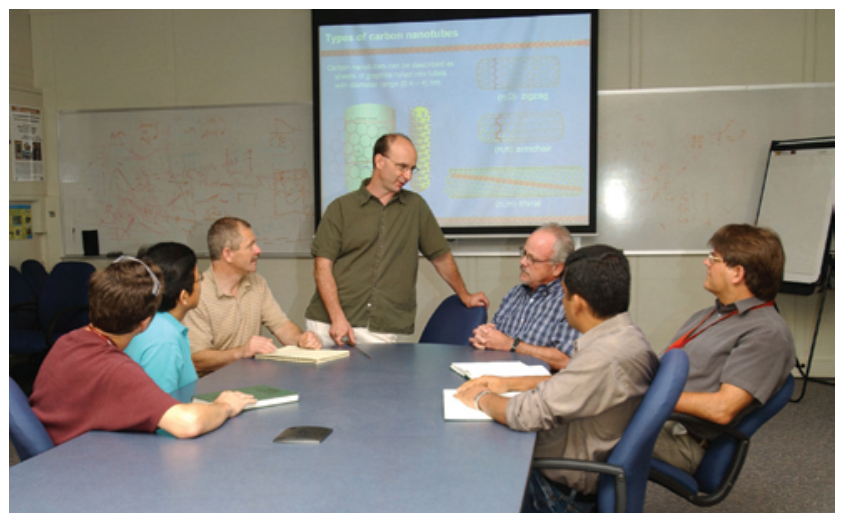

Carbon Nanotube Project Meeting

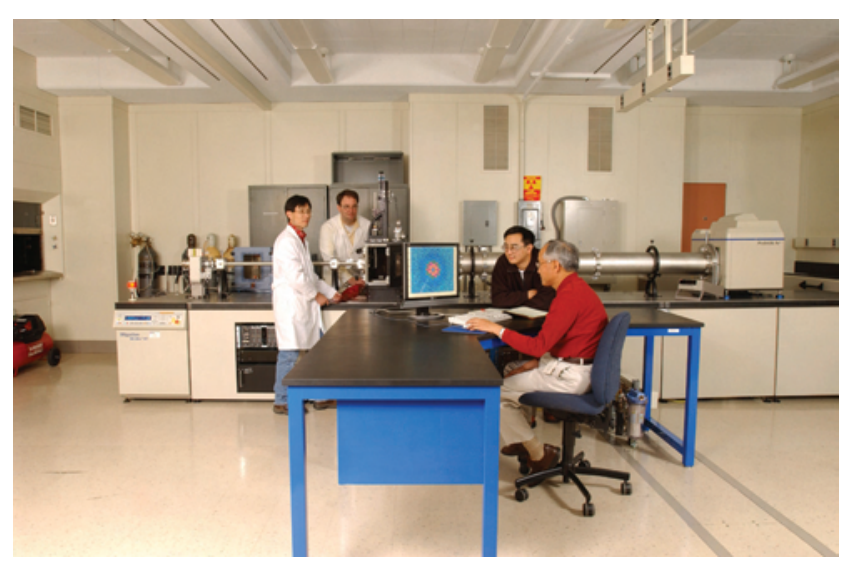

Polymers Division Small-Angle X-Ray Scattering Facility 


\section{Carbon Nanotube Materials:}

\section{Achieving The Gold Standard in Quality and Characterization}

The availability of high-quality, well-characterized carbon nanotube materials is essential to the development of advanced applications that exploit the nanotube's remarkable combination of properties. Our goal is to provide the methodologies that will produce Gold Standard nanotubes materials separated by length, diameter, and type. These high-quality samples will enable characterization of their electrical, optical and mechanical properties.

\section{Barry J. Bauer, Erik K. Hobbie and Kalman B. Migler}

\begin{abstract}
world-wide effort to harness the extraordinary Aproperties of carbon nanotubes and to develop revolutionary new functional applications ranging from high-speed electronics to biosensors, fuel cells, and displays is underway. However, typical nanotube materials contain dozens of different types of tubes, have a wide distribution of lengths, and frequently contain impurities. The ill-defined nature of this material prevents proper characterization, which hampers trade and application development.
\end{abstract}

Our goal is to enhance both the quality of nanotube materials and the characterization methodologies of them by the development of solution-based techniques which sort the nanotubes by type and length, and enable characterization on materials of high purity. We are currently focusing our efforts on three key areas which demonstrate the principles of our approach. First, we are developing metrics for assessing the dispersion of nanotubes in solutions and in polymers. Second, we are fractionating dispersed suspensions to produce nanotubes of well-defined length, and then characterizing the effects of length on their optical and toxicological properties. Third, we are laying the groundwork for the next phase of the project, using the length fractionated tubes to then separate by type, via combinations of flow and electric fields.

The characterization of nanotube dispersion is critical to our program, as only singly dispersed nanotubes can be separated by length and type. Further, fundamental characterizations through optical, electrical, and mechanical methods are sensitive to the degree of dispersion. We have compared a variety of optical, viscometric, and scattering techniques to measure dispersion, and found that DNA-wrapped nanotubes disperse well in aqueous suspensions, so we have chosen this material for the next phase of the project.
In the Technical Highlights section of this report, we describe our achievement of unprecedented dispersion using the DNA-wrapped nanotubes in a model polymer, and the resulting comparison of dispersion measurement techniques.

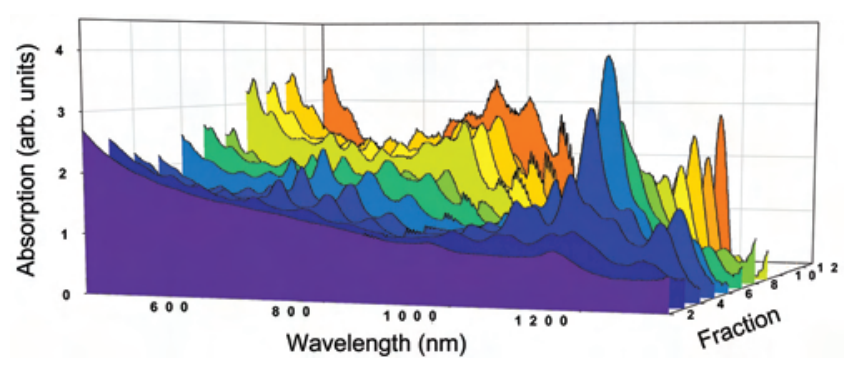

Figure 1: Absorption spectroscopy for 12 fractions that eluted from an ion-exchange column.

Next, we utilize the well-dispersed nanotubes for length fractionation through size-exclusion chromatography. We have introduced automated quality control measures into the chromatography via on-line viscometry and light scattering. We are exploring the effects of length on absorption and Raman spectroscopy, and we find a strong increase of absorbance with length as well as shifts in key Raman ratios. The well-characterized, length separated fractions are also being used in toxicology studies, and we find that the long SEC fractions show much lower toxicity.

Finally, we are laying the groundwork to take the length-separated tubes and further separate them by type (semi-conducting vs. metallic). This requires measures of the response of the DNA-wrapped nanotubes to electric fields and the complex flow fields in a fieldflow-fractionation system. We have measured a wide range of electrophoretic mobilites on tubes of the same nominal length, and have measured the polarizability and impedance of the tubes in $\mathrm{AC}$ electrical fields. The data generated will drive the design of future separation methods.

The methods and data developed here will be utilized to develop standards for nanotube characterization, and to develop complementary reference materials.

\section{Contributors and Collaborators}

V. Bajpai, M. Becker, J. Fagan, J. Obrzut, F. Phelan (Polymers Division, NIST); A. Hight Walker, I. Mandelbaum, J. Simpson (PL, NIST); A. Allen, V. Hackley (Ceramics Division, NIST); S. Hooker, W. Johnson; T. Yildirum (NIST Center for Neutron Research); S. Arepalli (NASA); M. Pasquali (Rice U.); B. Landi, R. Raffaelle(RIT) 


\section{Combinatorial Methods for Nanomaterials and Nanometrology}

Industrial developers of nanomaterials strive to adapt combinatorial and high-throughput methods to accelerate product discovery and optimization. This project addresses the primary barrier to the application of these attractive measurement approaches to nanostructured polymer materials: the design of appropriate combinatorial libraries. To meet this need, we produce and demonstrate library designs that express the factors governing nanomaterials behavior, and that are amenable to high-throughput characterization by industrially relevant nanometrologies.

\section{Michael J. Fasolka and Thomas H. Epps III}

$\mathrm{T}_{\mathrm{p}}^{\mathrm{k}}$ he structure and performance of nanostructured polymer materials, including self-assembling systems, nanocomposites, and lithographic resists, depend on a plethora of interdependent compositional, interfacial, and processing factors. This makes these materials prime candidates for combinatorial and high-throughput devel-opment. However, as detailed in a 2006 NIST Combinatorial Methods Center (NCMC) Industry Workshop Report, adaptation of combinatorial methods to these systems is hampered by the persistent challenge of de-signing appropriate combinatorial libraries (see NISTIR 7332, www.nist.gov/combi). Specifically, industry requires libraries that express the parameters relevant to nanomaterials behavior, and that can be analyzed with the measurements they currently rely upon, such as Transmission Electron Microscopy (TEM), Atomic Force Microscopy (AFM), and X-Ray Photoelectron Spectroscopy (XPS).

The NCMC has produced a suite of gradient combinatorial library designs that are readily employed for the high-throughput analysis of polymer nanomaterials. Gradients in temperature, composition, film thickness, and substrate surface energy $(\gamma)$ provide means to rapidly and comprehensively measure the effect of these key factors on structure and performance. Moreover, these planar libraries are highly geared for characterization by automated microscopy and spectroscopy methods. In 2006, we extended gradient approaches to analyze triblock copolymer thin films. In bulk systems, these materials self-assemble into intricate nanostructures that can be harnessed for membranes and electronic devices, but it is uncertain whether these compelling motifs persist in the thin film geometries required for these applications. We demonstrated a gradient design to gauge the effect of $\gamma$ on the film behavior of a triblock copolymer that exhibits a unique gyroid morphology in the bulk. As shown in Figure 1, our library revealed that this material switched from its bulk morphology to a lamellar motif in thin films, and illuminated a critical $\gamma$ (water contact angle of $\approx 70^{\circ}$ ) below which the material exhibited "autophobic dewetting." Automated XPS and NEXAFS (Near Edge X-Ray Absorption Fine Structure) analysis along the library shows that dewetting was driven by a $\gamma$-dependent structuring of a polymer monolayer that forms at the substrate surface. This work was reported via invited lectures at meetings of the American Physical Society and the American Chemical Society in 2006.

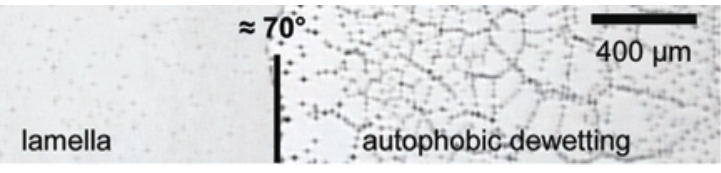

Figure 1: Optical micrograph of $\gamma$-dependent morphology and autophobic dewetting in a triblock copolymer thin film.

Also in 2006, we designed a method to prepare combinatorial libraries of film specimens for TEM analysis. The industrial need for this is paramount, since TEM is the primary tool many companies use for imaging nanostructures. As shown in Figure 2, our method employs an array of poly(acrylic acid) (PAA) droplets, which are deposited over a thin film library; in the Figure, a gradient library of block copolymer on silicon is shown. A polydimethylsiloxane (PDMS) gasket, with an array of holes, is used to sequester plugs of aqueous PAA solution while they dry atop the specimen. The system is then immersed in liquid nitrogen, which causes the PAA droplets to pull a library of 48 different film specimens from the substrate. Since they remain lodged in the gasket, the PAA plugs retain their registry. Samples are harvested by dissolving the PAA plugs to leave floating films that can be retrieved with TEM grids.

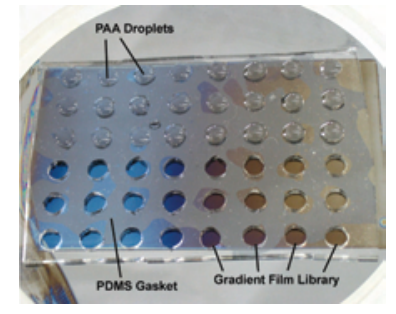

Figure 1: Combinatorial film libraries for TEM analysis.

\section{Contributors and Collaborators}

K. Roskov, D. DeLongchamp, A. Karim (Polymers Division, NIST); D. Fischer (Ceramics Division, NIST); E. Jablonski (Bucknell University); F. Bates (University of Minnesota) 


\section{Defects in Nanostructured Materials}

The functionality of nanoscale materials, critical to emerging technologies in data storage, microelectronics, and medical diagnostics, is strongly dependent on the density and location of physical, chemical, and magnetic defects. Currently, a lack of tools capable of characterizing defects in these materials limits the development of these important technologies. We are developing measurement platforms capable of high precision measurements of defects in nanostructured surfaces, and materials to enable manufacturing at these scales, as well as provide insight into materials properties that control defect formation.

\section{Ronald L. Jones and Alamgir Karim}

$M$ anufacturing reliable structures with nanometer scale dimensions is a potential route to advanced technologies for microelectronics, medial diagnostics and therapy, and data storage. However, manufacturing is challenged by the sensitivity of structural functionality to small concentrations of defects in size, shape, and chemical composition. Current methods of inspection are incapable of rapidly locating the "needle-in-ahaystack" defect until late in manufacturing stages, and are often destructive to the sample. We are developing measurement platforms capable of quantifying the type and number of defects, their location, and their resulting impact on materials properties.

In addition to advancing manufacturing methods, measurement platforms to characterize defects will advance the fundamental understanding of defect formation mechanisms, providing additional control over material properties. Controlled defect formation in bulk materials has long been an important route toward mechanical toughening in crystals, altering phase transitions in solids, and imposing the formation of amorphous glasses.

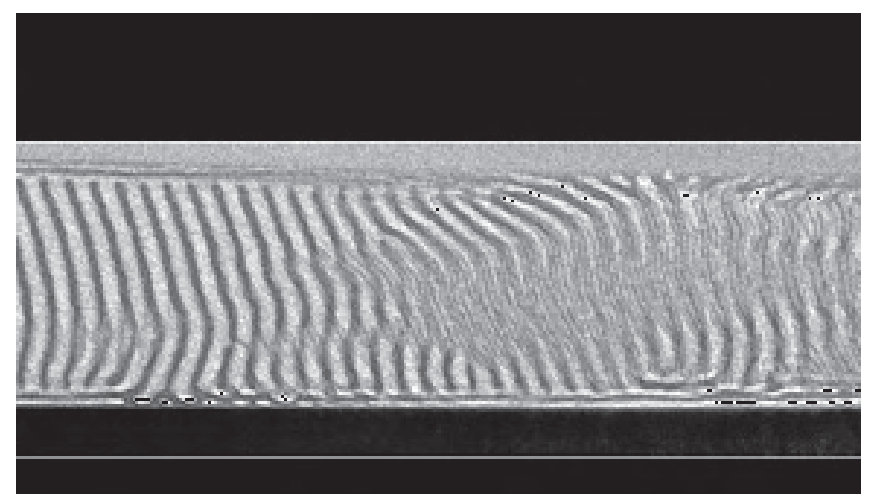

Figure 1: Transmission electron micrography of a cross sectioned porous silica film created by patterning with a block copolymer template. The image shows a wide range of structural irregularities (Image courtesy H.-C. Kim).

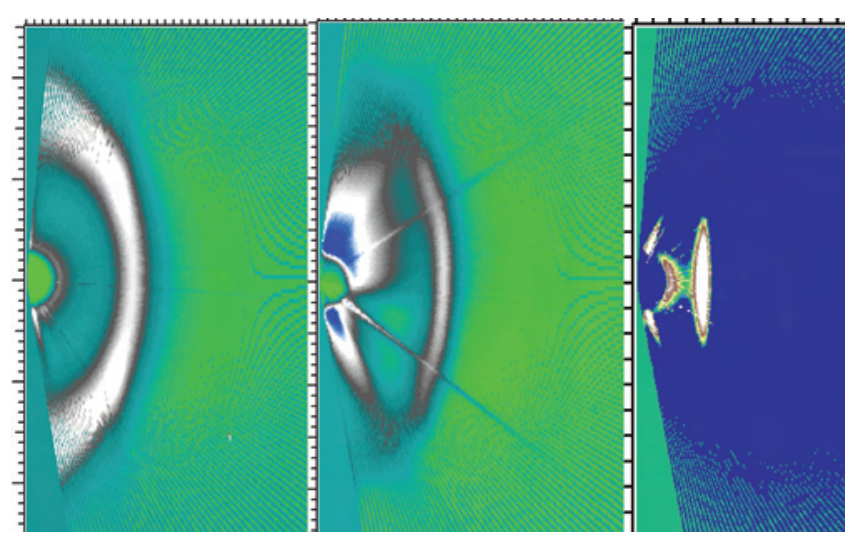

Figure 2: SAXS intensity as a function of scattering vector parallel (horizontal axis) and perpendicular to the substrate (vertical axis) for nanostructured silica films. The data shows qualitative and quantitative differences between varying degrees of orientational control, including (from left to right) randomly packed spheres, partially oriented cylinders, and highly oriented lamellae.

One platform under development uses small angle $\mathrm{x}$-ray scattering (SAXS) to measure the 3D distribution of orientation within nanostructured thin films. In Fig. 1 , the microphase structure of a block copolymer film has been chemically converted to a porous silica film as a potential route toward high density data storage and next generation membranes. Controlling the orientation of the morphology is critical to both applications. The data in Fig. 2 demonstrates both a qualitative and quantitative change in SAXS intensity as the orientation of the microphases is varied. Fits of the data provide high precision measures of the distribution in orientation non-destructively in a manner exportable to industry.

Other platforms being developed aim to characterize and quantify magnetic interactions in individual metallic nanoparticles, rapidly quantifying the concentration of defective particles for many applications, including medical diagnostics. Precise characterization of nanoparticle interactions is also aiding the development a "nanotagger" platform that utilizes nanoparticles to "seek, identify, and locate" chemical and topological defects on surfaces by attaching and fluorescing. Applications of these platforms focus on fundamental understanding of packing defects during self-assembly of polymer crystals and block copolymer films.

\section{Contributors and Collaborators}

J. Benkoski, B. Berry, S. Lacerda, J. F. Douglas (Polymers Division, NIST); J. Pyun (University of Arizona); S. Kim, R. Briber (University of Maryland); T. Albrecht (Hitachi); K.-W. Choi (Intel); K.-I. Nihara, H. Jinnai (Kyoto Institute of Technology); H.-C. Kim (IBM Almaden) 


\section{Mechanics of Complex Interfaces: Libraries for Probing Interfacial Adhesion}

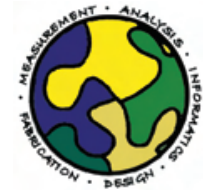

Technologies such as next-generation electronics, advanced adhesives, and functional coatings depend on robust interfaces between a variety of hard and soft materials. To ensure the reliability of new products and drive these products into new and emerging markets, industry requires means to assess the host of factors that govern the mechanical behavior of these systems. With an emphasis on combinatorial and high-throughput approaches, this project develops and demonstrates innovative tools for measuring the mechanical properties and performance of materials systems that incorporate complex interfaces.

\section{Christopher M. Stafford}

Roughness plays a critical role in interfacial adhesion, however systematic measurements of the effect of this factor are difficult to achieve. To meet this need, we are developing approaches to design roughness libraries that span multiple length scales. For example, a versatile method for creating roughness gradients exploits the melting and fusion of surface-adsorbed polymer microspheres. As shown in Figure 2, the scale of roughness is gradually varied by melting the spheres on a temperatures gradient. In addition, we are harnessing a buckling phenomenon to design libraries that independently vary the amplitude and lateral spacing of surface roughness.
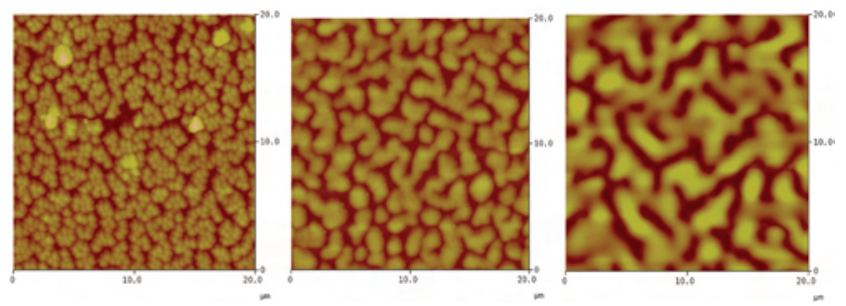

Figure 2: Atomic force micrographs of a roughness library produced by melting polymer microspheres on a temperature gradient.

Development of multi-component adhesives and coatings by industry involves testing hundreds or thousands of candidate formulations. To address this challenge, we are developing routes to prepare compositional libraries of the viscous polymer mixtures that are inherent to these systems. This work is part of a NIST Combinatorial Methods Center (NCMC) Focus Project with Intel and ICI/National Starch, completed in 2006. These companies will use these libraries for the highthroughput optimization of epoxy underfill materials for flip-chip electronics. In 2007, we will develop and demonstrate the rapid measurement of the interfacial strength across these libraries using our combinatorial edge lift-off test.

Polymer brushes provide a facile route for expressing diverse chemistry and functionality on a surface. As illustrated in Figure 1, by changing monomer types, surface initiator site density, and reaction times across a surface, we are producing libraries that systematically express chemical moieties, chain lengths, and molecular architectures, which dictate polymer adhesion. These types of libraries also present a unique opportunity to study the effects of molecular rearrangements at contacting interfaces.

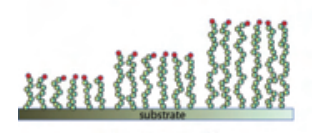

chain length

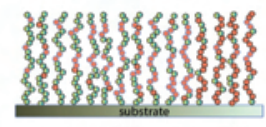

chemical functionality

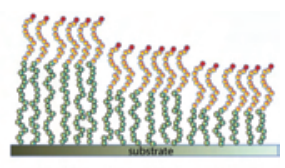

molecular architecture
Figure 1: Illustration of different polymer brush libraries for assessing interfacial adhesion.
In 2006, we reported our project achievements at key national conferences in the adhesion field. Invited lectures on this work were given at the two Gordon Research Conferences (Science of Adhesion, and ComAdhesion Society, and the Materials Research Society. We also disseminated our results to industrial partners at semi-annual NCMC Workshop lectures.

\section{Contributors and Collaborators}

C.M. Stafford, H. Huang, X. Hu, J.H. Kim, J.Y. Chung, M.Y.M. Chiang, C. Xu, K.L. Beers (Polymers Division, NIST); R. Huang (UT Austin); Y. Dar, S. Gibbon (ICI/National Starch); N. Patel (Intel) binatorial and High-Throughput Material Science), the 


\section{Nanoimprint Lithography}

Nanoimprint lithography is a high-throughput patterning technique with the ability to directly replicate features smaller than $10 \mathrm{~nm}$, not just in optimized resists, but also in functional materials. This makes the technology extremely attractive for many forms of nanotechnology, starting with CMOS applications, and extending into many emerging technologies. To fully harness the potential of nanoimprint lithography requires development of the measurement infrastructure to evaluate, quantify, and tailor the unique properties that come with patterning materials on the nanoscale.

\section{Christopher L. Soles}

$\mathrm{T}$ here are two aspects of nanoimprint lithography introducing measurement challenges. The first aspect is resolution. Because this technique has inherently high-resolution, basic shape metrology becomes a significant challenge. Quantifying physical dimensions is critical for evaluating patterning processes, quality control, and assessing the long-term stability of nanoscale features. Currently the semiconductor industry is challenged by with shape metrology at the $65 \mathrm{~nm}$ technology node. The potential jump to sub-10 nm features exacerbates this challenge. The second aspect stems from directly patterning the material of interest. This differs from other lithographies where a sacrificial resist transfers the pattern into the functional material. Beyond pattern shape, it then becomes critical to quantify how the desired properties of the functional material are impacted by the imprint process. This is a new class of metrology for the nanofabrication community.

Significant progress has been made on both aspects. Critical dimension small angle x-ray scattering (CDSAXS) and specular $x$-ray reflectivity (SXR) for pattern shape characterization were adapted for nanoimprint lithography. Papers were published describing how these techniques could be used to quantify, with nm precision, the fidelity of the imprint pattern transfer process. If the nanostructures are to be made of functional material, they must also be stable for long periods of time. Stability in nanostructures, especially polymeric ones, will be a major challenge for nanomanufacturing. Using CDSAXS and SXR, we found that the imprint process can induce internal stress that can compromise the stability of the resulting nanostructures. By modifying imprint variables, such as the time, temperature, and the molecular mass of the material being imprinted, we illustrated how these stresses can be minimized.
Examples of imprinted functional materials include semicrystalline materials where the crystal morphology needs to be controlled for organic semiconductor applications, and nanoporous low-k dielectric materials for interconnect technologies. For the patterned low-k materials, the way in which the imprint process affects the porosity will be important for controlling the effective $\mathrm{k}$ of the pattern. The Figure below, obtained by SXR and CD-SAXS, compares the imprinted pattern cross-section to the mold, quantifying the effects of pattern shrinkage upon vitrification. A modest shrinkage occurs in the vertical direction, with almost no shrinkage in the lateral. Knowing and controlling this shrinkage will be critical for next generation devices. The panel to the right further shows how the porosity of the pattern varies as a function of height (obtained by x-ray porosimetry). The porosity is reduced in the patterned region. These types of metrologies will be critical for developing effective imprint process for low-k materials

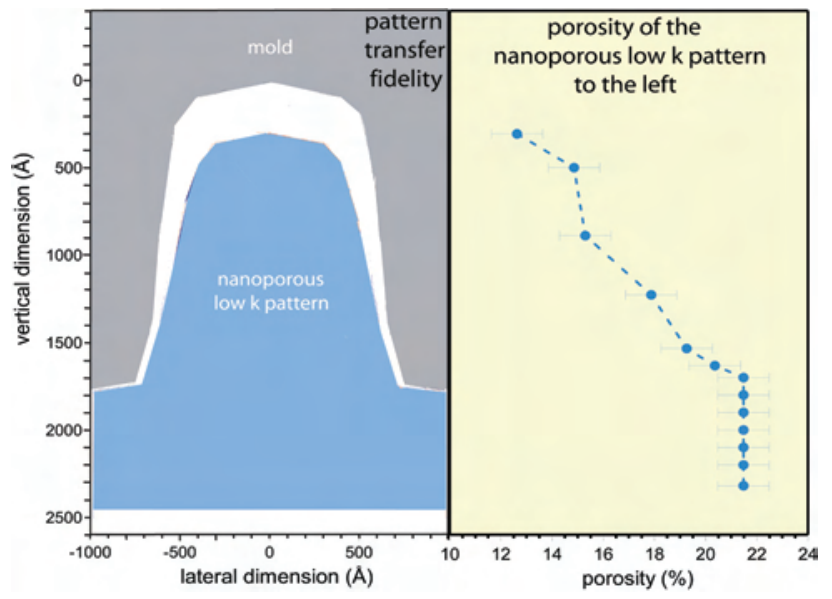

This year our findings were published in high profile journals, including Applied Physics Letters, Nano Letters, and Physical Review Letters. Industrial outreach to the semiconductor industry was achieved through invited and contributed presentations at major lithography and metrology conferences. These included a Webcast for Semiconductor International that was viewed by thousands worldwide. Bilateral cooperative agreements were established and funded with Seoul National University and the Indian Institute of Technology, Kanpur.

\section{Contributors and Collaborators}

H.-J. Lee, H. Ro, Y. Ding, B. Okerberg, K. Alvine, R. Jones, E. Lin, A. Karim, W.-L. Wu (Polymers Division, NIST); D. Hines (University of Maryland); S. Pang, D. Gidley (University of Michigan) 


\section{Self-Assembly for Soft Nanomanufacturing}

The self-assembly of molecules and nanoparticles is central to the formation of biological structures, and the synthetic emulation of this process offers great promise for nanometrology and nanofabrication. Accordingly, this approach to fabrication has recently been identified in U.S. chemical industry roadmaps for nanomanufacturing. We respond to this challenge by developing theoretical, computational, microfluidic, and optical methods to enable precision measurements of the thermodynamic and kinetic parameters controlling synthetic self-assembly

\section{Jack F. Douglas and Steven D. Hudson}

$\mathrm{S}_{\mathrm{t}}^{\mathrm{e}}$ elf-assembly of nanoparticles into functional structures such as sensors, optical filters, and pharmaceutical agents holds great promise for inexpensive manufacturing at the nanoscale. The greatest opportunity involves self-assembly of highly directional and specific interactions that lead to structures that have a chain-like, sheet-like, or shell-like structure. The basic design rules that describe how a given set of anisotropic particles assembles into a given structure are now being developed as one component of this project. The second component addresses the critical need for methods to measure these anisotropic and specific interactions between particles; such basic information is the key to determining how the asymmetry of interacting particles encodes information about the geometry of the organized structure.

Our theoretical approach to the principles governing this process is inspired by existing models and measurements for the self-assembly of actin, tubulin, and the ubiquitous icosahedral shell structures of viral capsids. In our simulations, we introduce a family of simple, yet anisotropic, potentials that give rise to the self-assembly of linear polymeric, random surface ("membrane”), tubular ("nanotube"), and hollow icosahedral structures that are similar in many respects to their biological counterparts, yet simple enough for model experimental realization. The potentials involve equivalent particles and an interplay between directional (dipolar, multipolar) and short range (van der Waals) interactions. Specifically, we find that the dipolar potential, having a continuous rotational symmetry about the dipolar axis, gives rise to chain formation, while particles with multipolar potentials having discrete rotational symmetries (square quadrupole or triangular ring of dipoles or "hexapole") lead to the self-assembly of sheet (see
Figure), nanotube, and hollow icosahedral geometries. These examples reveal a general design rule: the local rotational symmetries of the organizing particles tend to be preserved in the final organized structure. They also strongly motivate the need for methods to measure the anisotropic interaction strength. We also show that fluctuations in the assembly kinetics and the form of assembly and polymorphism can be controlled with appropriate seeding of the assembly process. Seeding is thus crucial to the effective control of kinetics and form of synthetic self-assembly.

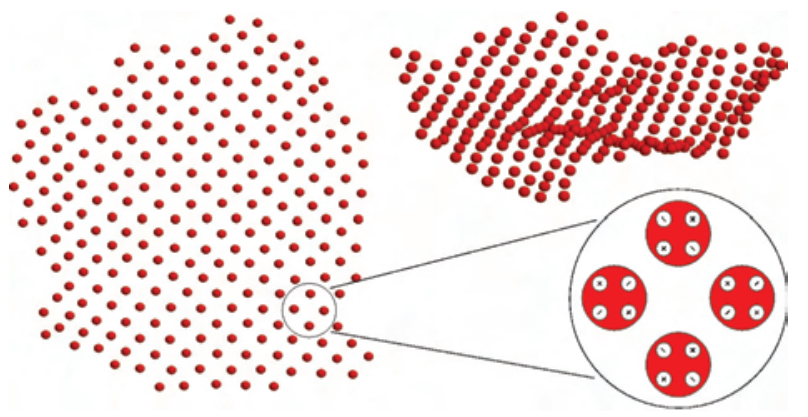

Figure 1: Self-assembled sheet preserving the local symmetry of quadrupole particles (shown in enlargement).

Extensive effort has also been made in characterizing the thermodynamic nature of self-assembly, and in the numerical and experimental procedures required in determining the thermodynamic parameters governing these transitions. A recent commentary in Science magazine (July 7, p.55) highlights this modeling effort.

Current method and technology development here explores the potential of particles with charged patches or magnetic moments to control assembly. Surfacesensitive and patterning techniques are being adapted to access anisotropic interactions between particles. These measurements employ the asymmetry inherent at a surface.

The geometry of these assemblies is characterized by electron microscopy, microfluidic, and optical methods. Recently, we have discovered hollow colloidal shell structures, and are working with Percec and coworkers to manipulate the interactions controlling this structure. Microfluidic traps have been used to determine interaction strength within assemblies.

\section{Contributors and Collaborators}

T. Chastek (Polymers Division, NIST); V. Hackley (Ceramics Division, NIST); Y. Xia (U. Washington); V. Percec (U. Pennsylvania); K.F. Freed and J. Dudowicz (U. Chicago); S. Kumar (Rensselaer Polytechnic Institute); W. Losert (U. Maryland); S. Velankar (U. Pittsburgh) 



\section{Safety and Reliability}

We take for granted that the physical infrastructure around us will perform day in and day out with consistent reliability. Yet, failures occur when these structures degrade to where they no longer sustain their design loads, or when they experience loads outside their original design considerations. In addition, we have become increasingly aware of our vulnerability to intentional attacks. The Safety and Reliability Program within the NIST Materials Science and Engineering Laboratory (MSEL) was created to develop measurement technology to clarify the behavior of materials under extreme and unexpected loadings, to assess integrity and remaining life, and to disseminate guidance and tools to assess and reduce future vulnerabilities. Project selection is guided by identification and assessment of the particular vulnerabilities within our materials-based infrastructure, and focusing on those issues that would benefit strongly by improved measurements, standards, and materials data. Ultimately, our goal is to moderate the effects of acts of terrorism, natural disasters, or other emergencies, all through improved use of materials. Our vision is to be the key resource within the Federal Government for materials metrology development as realized through the following objectives:

- Develop advanced measurement methods needed by industry to address reliability problems that arise with the development of new materials;

- Develop and deliver standard measurements and data;

- Identify and address vulnerabilities and needed improvements in U.S. infrastructure; and

- Support other agency needs for materials expertise.

Contact: Chad R. Snyder

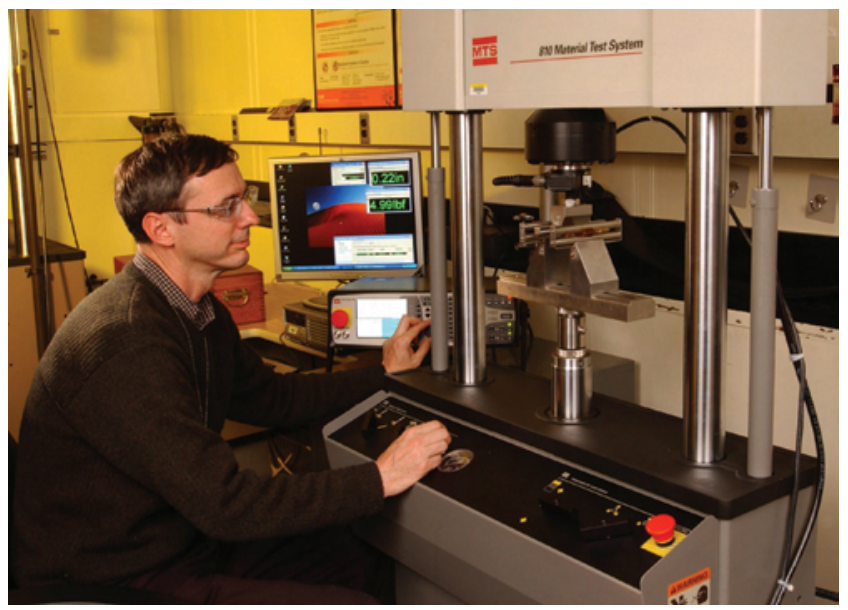




\section{Ballistic Resistance of Polymeric Materials}

This project is developing metrologies and Failure of a first responder's personal body armor prompted NIST's Office of Law Enforcement Standards (OLES), under the auspices of the National Institute of Justice (NIJ), to initiate a program to assess the long-term durability and effectiveness of current and future soft body armor products. In response, we are developing metrologies and models to test and predict the long-term reliability of polymers used in these products.

\section{Gale A. Holmes and Chad R. Snyder}

$\mathrm{I}_{\mathrm{n}}^{\mathrm{n}}$ a recent review [1] of the potential degradation mechanisms of the ballistic fiber poly (p-phenylene benzobisoxazole, i.e., $\mathrm{PBO}$ ), ultraviolet (UV) radiation exposure, exposure to moisture, elevated temperature exposure, and mechanical folding have been identified as mechanisms that may compromise the structural integrity of the active ballistic fiber during use. Since answers related to the long-term durability of the armor are sought, any testing methodology must allow relevant degradation mechanisms to occur under field conditions and controlled exposure conditions without compromising the structural integrity of the armor. As a result the testing methodology must be non- or minimally-invasive, precisely determine the mechanical properties of the ballistic fibers, and reflect the ballistic performance properties of the armor.

ASTM Standard D3379-75, a statistically robust testing methodology that measures the tensile strength and Young's modulus for high modulus single-filament materials that also addresses the issue of sample size, was modified to accurately measure the strain-to-failure and diameter of single filaments, with the latter providing a more accurate measure of the yield strength and Young's modulus of the filament. This test was designed to monitor changes in fiber properties without compromising the integrity of flexible body armor. In addition to showing that changes in ballistic fiber properties are readily quantified using this test, results from $\mathrm{PBO}$ filaments have shown that deviations of the yield strength data from the expected normal distribution can be attributed to abnormal variations in the fiber diameter of the PBO filament along its length, thus highlighting the need to profile the length of the tested fiber.

Research data from 2005 indicated that a single severe fold in a PBO fiber could result in a greater than $10 \%$ reduction in ballistic performance, as quantified by the test method discussed above. To better quantify the impact of fatigue on ballistic performance, a device was designed (below) for use on a servo hydraulic machine, such as those commonly used to measure the fatigue resistance of structural materials, to fold single and multiple layers of woven fabric of ballistic material. Initial data from this device indicate that repeated folding of PBO fibers can result in a greater than $20 \%$ reduction in ballistic performance after 5000 cycles or seven months of wear! Since this is a new testing methodology, research is being conducted to validate these preliminary test results.

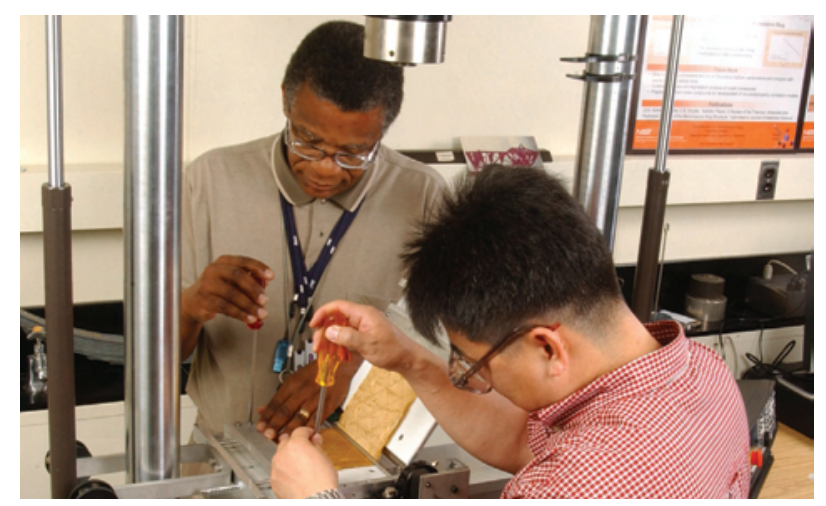

Our research on the degradation pathways of PBO and PBO-like materials has focused on the role of residual phosphoric acid on the chemical degradation of these materials. A procedure was devised to extract residual acid using a soxhlet extractor, methylate any extracted phosphoric acid by diazomethane, and identify and quantify its presence by gas chromatography-mass spectrometry. This more involved procedure was devised to overcome the presence of phosphorus containing sizing material that may be added during processing. Initial results indicate that after a week-long extraction process, only $25 \%$ of the phosphorus containing species was removed from the fiber. Research is ongoing to identify the extracted phosphorus, which is presumed to be composed primarily of the sizing additive, and to devise a more robust approach for removing the remaining phosphorus believed to be trapped in the micro and nanoscale voids of the fiber structure.

\section{References}

1. G. A. Holmes, K. Rice, C.R. Snyder, Journal of Materials Science 41, 4105 (2006).

\section{Contributors and Collaborators}

J. Kim, W.G. McDonough, W. Blair, K. Flynn, C.M. Guttman, W. Wallace (Polymers Division, NIST); K. Rice (Office of Law Enforcement Standards, NIST); J.R. Sieber (Analytical Chemistry Division, NIST)K. Rice (Office of Law Enforcement Standards, EEEL); T. Dang (Air Force Office of Scientific Research) 


\section{Polymers Division FY06 Annual Report Publication List}

\section{Polymer Science and Characterization}

\section{Dielectric Spectroscopy}

J. Obrzut, "Coaxial line reflection method for dielectric permittivity of thin film samples at microwave frequencies - numerical and experimental analysis," in Proceedings of the 40th Annual Symposium of the International Microwave Power Institutes, 2006, Boston, MA.

J. Obrzut, "Measurement of Materials Dielectric Properties" in Handbook of Materials Measurement Methods edited by H. Czichos, T. Saito and L. Smith, (Springer, 2006).

J. Obrzut and R. Nozaki, “ TDR Permittivity Measurements Of Dielectric Films," in Procceedings of the IEEE Conference on Instrumentation and Measurement. April 2006, Sorrento, Italy.

Anopchenko, T. Psurek, D. VanderHart, J. F. Douglas, and J. Obrzut, "Dielectric Study of the Antiplasticization of Trehalose by Glycerol," Physical Review E 74, (2006).

\section{Ballistic Resistance of Polymers}

G. A. Holmes, K. Rice, and C. R. Snyder, "Ballistic Fibers: A Review of the Thermal, Ultraviolet and Hydrolytic Stability of the Benzoxazole Ring Structure," Journal of Materials Science 41, 4105-4116 (2006).

\section{Polymer Mass Spectrometry}

W. E. Wallace, M. A. Arnould, and R. Knochenmuss, "2,5-Dihydroxybenzoic Acid: Laser Desorption/Ionisation As a Function of Elevated Temperature," International Journal of Mass Spectrometry 242, 13-22 (2005).

S. J. Wetzel, C. M. Guttman, K. M. Flynn, and J. J. Filliben, "Significant Parameters in the Optimization of MALDI-TOF-MS for Synthetic Polymers," Journal of the American Society for Mass Spectrometry 17, 246-252 (2006).

C.M. Guttman, “Mass Spectrometry,” in Encyclopedia of Polymer Science and Technology, Concise, third edition, edited by H. F. Mark, (Wiley, 2007).

W.E. Wallace, C.M. Guttman, K.M. Flynn, and A.J. Kearsley, "Development of an Absolute Molecular Mass Distribution Polymer Standard," in Abstracts of the Third International Symposium on Separation and Characterization of Natural and Synthetic Macromolecules, Amsterdam, Netherlands (2006).

\section{Rheology and Processing}

D. Bigio, M. G. Meillon, S. B. Kharchenko, D. Morgan, H. Zhou, S. R. Oriani, C. W. Macosko, and K. B. Migler, "Coating Kinetics of Fluoropolymer Processing Aids for Sharkskin Elimination: The Role of Droplet Size," Journal of Non-Newtonian Fluid Mechanics 131, 22-31 (2005).

A.J. Bur and K. Migler, “A Multi-Sample Melt Micro-Rheometer," in Proceedings Of the Annual Technical Meeting of the Society of Plastics Engineers (ANTEC), May, 2006, Charlotte, NC.

\section{Polymer Theory}

J. Dudowicz, K. F. Freed, and J. F. Douglas, “The Glass Transition Temperature of Polymer Melts," Journal of Physical Chemistry B 109, 21285-21292 (2005).

J. Dudowicz, K. F. Freed, and J. F. Douglas, "Fragility of Glass-Forming Polymer Liquids,” Journal of Physical Chemistry B 109, 21350-21356 (2005).

J. Dudowicz, K. F. Freed, and J. F. Douglas, “Direct Computation of Characteristic Temperatures and Relaxation Times for Glass-Forming Polymer Liquids," Journal of Chemical Physics 123, 111102 (2005).

B. M. Besancon, C. L. Soles, and P. F. Green, "Glass Transition of Miscible Binary Polymer-Polymer Thin Films," Physical Review Letters 97, 057801 (2006).

J. F. Douglas, J. Dudowicz, and K. F. Freed, "Does Equilibrium Polymerization Describe the Dynamic Heterogeneity of Glass-Forming Liquids?" Journal of Chemical Physics 125, 144907 (2006).

J. Dudowicz, K. F. Freed, and J. F. Douglas, “Entropy Theory of Polymer Glass Formation Revisited. I. General Formulation,” Journal of Chemical Physics 124, 064901 (2006).

A. Warren, J. T. Cabral, and J. F. Douglas, "Solution of a Field Theory Model of Frontal Photopolymerization," Physical Review E 72, 021801 (2005).

L. Granasy, T. Pusztai, T. Borzsosnyi, G. I. Toth, G. Tegze, J. A. Warren, and J. F. Douglas, "Polycrystalline Patterns in Far-From-Equilibrium Freezing: a Phase Field Study," Philosophical Magazine 86, 3757-3778 (2006). 
L. Granasy, T. Pusztai, T. Borzsonyi, G. Toth, G. Tegze, J. A. Warren, and J. F. Douglas, "Phase Field Theory of Crystal Nucleation and Polyerystalline Growth: A Review,” Journal of Materials Research 21, 309-319 (2006).

JL. Granasy, T. Pusztai, G. Tegze, J. A. Warren, and J. F. Douglas, "Growth and Form of Spherulites," Physical Review E 72, 011605 (2005).

K. Rah, K. F. Freed, J. Dudowicz, and J. F. Douglas, "Lattice Model of Equilibrium Polymerization. V. Scattering Properties and the Width of the Critical Regime for Phase Separation," Journal of Chemical Physics 124, 144906 (2006).

R. A. Riggleman, K. Yoshimoto, J. F. Douglas, and J. J. de Pablo, "Influence of Confinement on the Fragility of Antiplasticized and Pure Polymer Films," Physical Review Letters 97, 045502 (2006).

S. K. Kumar, G. Szamel, and J. F. Douglas, “Nature of the Breakdown in the Stokes-Einstein Relationship in a Hard Sphere Fluid,” Journal of Chemical Physics 124, 214501 (2006).

H. Zhang, D. J. Srolovitz, J. F. Douglas, and J. A. Warren, "Characterization of Atomic Motion Governing Grain Boundary Migration,” Physical Review B 74, 115404 (2006).

\section{Biomaterials}

\section{Metrology for Biomechanical Effects on Cell-Material Interactions}

L.M. Pakstis, L.A. Henderson, and J.P. Dunkers, "Physical and Biological Characterization of Protein Modified Biomaterial Surfaces," in Proceedings of the New Jersey Center for Biomaterials 8th Annual Biomaterials Symposium, 2006, New Brunswick, NJ.

K. M. N. Oates, W. E. Krause, R. L. Jones, and R. H. Colby, "Rheopexy of Synovial Fluid and Protein Aggregation,” Journal of the Royal Society Interface 3, 167-174 (2006).

\section{Metrology for 3D Scaffold Characterization}

K. Zhang, N. R. Washburn, J. M. Antonucci, and C. G. Simon, "In Vitro Culture of Osteoblasts With Three Dimensionally Ordered Macroporous Sol-Gel Bioactive Glass (3DOM-BG) Particles," Bioceramics 17, 655-658 (2005).
S. Lin-Gibson, R. L. Jones, N. R. Washburn, and F. Horkay, "Structure-Property Relationships of Photopolymerizable Poly(Ethylene Glycol) Dimethacrylate Hydrogels," Macromolecules 38, 2897-2902 (2005).

S. Lin-Gibson, S. Bencherif, J. M. Antonucci, R. L. Jones, and F. Horkay, "Synthesis and Characterization of Poly(Ethylene Glycol) Dimethacrylate Hydrogels," Macromolecular Symposia 227, 243-254 (2005).

T. W. Kee and M. T. Cicerone, "Biological Imaging Using Broadband Coherent Anti-Stokes Raman Scattering (CARS) Microscopy," Biophysical Journal 88, 362A (2005).

N. P. Patel, M. A. Hunt, S. Lin-Gibson, S. Bencherif, and R. J. Spontak, "Tunable CO2 Transport Through Mixed Polyether Membranes," Journal of Membrane Science 251, 51-57 (2005).

M. Y. M. Chiang, X. F. Wang, F. A. Landis, J. Dunkers, and C. R. Snyder, "Quantifying the Directional Parameter of Structural Anisotropy in Porous Media," Tissue Engineering 12, 1597-1606 (2006).

J.P. Dunkers, J.G. Hagedorn, A. Peskin, J.T. Kelso, and J.E. Terrill, "Quantitative, Interactive Measurement of Tissue Engineering Scaffold Structure Using Immersive Visualization," in Proceedings of the 8th NJ Symposium on Biomaterials, New Brunswick, NJ (2006).

J.P. Dunkers, J.G. Hagedorn, A. Peskin, J.T. Kelso, J.E. Terrill, and L. Henderson, "Interactive, Quantitative Analysis Of Scaffold Structure Using Immersive Visualization," in Proceedings of BIO2006, Amelia Island, FL (2006).

J.P. Dunkers, S.D. Leigh, M.T. Cicerone, and L. Henderson, "Quantifying The Structure Of Candidate Geometric Reference Scaffolds," in Regenerate 2006, Pittsburgh, PA (2006).

T. Dutta Roy, J.S. Stephens, A. Rege, M.T. Cicerone, J. Kohn, and M.L. Becker, "Osteoblast Response on Tyrosine-Derived Polycarbonate Tissue Engineering Scaffolds,” in Biophysical Journal, New Brunswick, NJ (2006).

T. Dutta Roy, J.J. Stone, W. Sun, E.H. Cho, S.J. Lockett, F.W. Wang, and L. Henderson, "Colocalization Of Cell Adhesion Proteins On 3D Tissue Engineering Scaffolds Fabricated By Rapid Prototyping,” in Transactions of the Society for Biomaterials 31st Annual Meeting and Exposition, 2006, Pittsburgh, PA .

T. Dutta Roy, J.J. Stone, W. Sun, E.H. Cho, S.J. Lockett, F.W. Wang, and L. Henderson, "Expression Of Osteoblast Adhesion Proteins On 3D Rapid Prototyped Scaffolds," in Transactions of the 52nd Annual Meeting of the Orthopaedic Research Society, Chicago, IL. 
J. A. Cooper, L. O. Bailey, J. N. Carter, C. E. Castiglioni, M. D. Kofron, F. K. Ko, and C. T. Laurencin, "Evaluation of the Anterior Cruciate Ligament, Medial Collateral Ligament, Achilles Tendon and Patellar Tendon As Cell Sources for Tissue-Engineered Ligament," Biomaterials 27, 2747-2754 (2006).

T. W. Kee, H. X. Zhao, and M. T. Cicerone, "OneLaser Interferometric Broadband Coherent Anti-Stokes Raman Scattering," Optics Express 14, 3631-3640 (2006).

Y. Hotta, K. Komatsu, and F.W. Wang, "Cure Monitoring Of Hydrosilation-Curable Silicones By Fluorescence Spectroscopy," in Proceedings of Society For Biomaterials 31st Annual Meeting, 2006, Pittsburgh.

F. A. Landis, J. S. Stephens, J. A. Cooper, M. T. Cicerone, and S. Lin-Gibson, "Tissue Engineering Scaffolds Based on Photocured Dimethacrylate Polymers for in Vitro Optical Imaging,” Biomacromolecules 7, 1751-1757 (2006).

C. T. Laurencin, Y. Khan, M. Kofron, S. El-Amin, E. Botchwey, X. J. Yu, and J. A. Cooper, "Tissue Engineering of Bone and Ligament - A 15-Year Perspective," Clinical Orthopaedics and Related Research 221-236 (2006).

W. J. Li, J. A. Cooper, R. L. Mauck, and R. S. Tuan, "Fabrication and Characterization of Six Electrospun Poly(Alpha-Hydroxy Ester)-Based Fibrous Scaffolds for Tissue Engineering Applications," Acta Biomaterialia 2, 377-385 (2006).

S. Lin-Gibson, F. A. Landis, and C. M. Stafford, "Polymerization Shrinkage Measurements of PhotocrossLinked Dimethacrylate Films," Abstracts of Papers of the American Chemical Society 231 (2006).

S. Lin-Gibson, F. A. Landis, and P. L. Drzal, "Combinatorial Investigation of the Structure-Properties Characterization of Photopolymerized Dimethacrylate Networks,” Biomaterials 27, 1711-1717 (2006).

\section{Metrology for Dental Materials}

J. M. Antonucci, S. H. Dickens, B. O. Fowler, H. H. K. Xu, and W. G. McDonough, "Chemistry of Silanes: Interfaces in Dental Polymers and Composites," Journal of Research of the National Institute of Standards and Technology 110, 541-558 (2005).

E. A. Wilder and J. M. Antonucci, "Improved Dental Composites Utilizing Dibenzylidene Sorbitol Networks,” Macromolecular Symposia 227, 255-263 (2005).
D. Skrtic, S. Y. Lee, J. M. Antonucci, and D. W. Liu, "Amorphous Calcium Phosphate Based Polymeric Composites: Effects of Polymer Composition and Filler's Particle Size on Composite Properties," Bioceramics 17, 737-740 (2005).

Dundigalla, S. Lin-Gibson, V. Ferreiro, M. M. Malwitz, and G. Schmidt, "Unusual Multilayered Structures in Poly(Ethylene Oxide)/Laponite Nanocomposite Films," Macromolecular Rapid Communications 26, 143-149 (2005).

J. M. Antonucci and D. Skrtic, "Matrix Resin Effects on Selected Physicochemical Properties of Amorphous Calcium Phosphate Composites," Journal of Bioactive and Compatible Polymers 20, 29-49 (2005).

J. Antonucci, T. B. Icenogle, W. F. Regnault, D. W. Liu, J. N. R. O'Donnell, and D. Skrtic, "Polymerization Shrinkage and Stress Development in Bioactive Urethane Acrylic Resin Composites," Abstracts of Papers of the American Chemical Society 231 (2006).

E. F. Burguera, H. H. K. Xu, and M. D. Weir, "Injectable and Rapid-Setting Calcium Phosphate Bone Cement With Dicalcium Phosphate Dihydrate," Journal of Biomedical Materials Research Part B-Applied Biomaterials 77B, 126-134 (2006).

N. Eidelman, N. Tsutsumi, and C. K. Chiang, "Micro FTIR Mapping of Nanometer Ferroelectric Polymer Films," Macromolecular Rapid Communications 27, 558-562 (2006).

M. Farahani, W. E. Wallace, J. M. Antonucci, and C. M. Guttman, "Analysis by Mass Spectrometry of the Hydrolysis/Condensation Reaction of a Trialkoxysilane in Various Dental Monomer Solutions," Journal of Applied Polymer Science 99, 1842-1847 (2006).

W.F. Regnault, T.B. Icenogle, J.M. Antonucci, D.W. Liu, and D. Skrtic, "Structure/Property Relationships In Urethane Dimethacrylate-Based Acp Composites,” in Proceedings of the 2006 Annual Meeting of the Society for Biomaterials, Pittsburgh, PA.

J. N. R. O'Donnell, D. Skrtic, and J. M. Antonucci, "Amorphous Calcium Phosphate Composites With Improved Mechanical Properties," Journal of Bioactive and Compatible Polymers 21, 169-184 (2006).

D. Skrtic, J. M. Antonucci, and D. W. Liu, "Ethoxylated Bisphenol Dimethacrylate-Based Amorphous Calcium Phosphate Composites,” Acta Biomaterialia 2, 85-94 (2006).

F. W. Wang and R. E. Lowry, "Wavelength-Shift Fluorescent Probes for Monitoring of Polymerization," Journal of Applied Polymer Science 101, 747-750 (2006). 
M. D. Weir, H. H. K. Xu, and C. G. Simon, "Strong Calcium Phosphate Cement-Chitosan-Mesh Construct Containing Cell-Encapsulating Hydrogel Beads for Bone Tissue Engineering,” Journal of Biomedical Materials Research Part A 77A, 487-496 (2006).

K. S. Wilson and J. M. Antonucci, "Interphase Structure-Property Relationships in Thermoset Dimethacrylate Nanocomposites," Dental Materials 22, 995-1001 (2006).

H. H. K. Xu, L. Sun, M. D. Weir, J. M. Antonucci, S. Takagi, L. C. Chow, and M. Peltz, "Nano DCPAWhisker Composites With High Strength and $\mathrm{Ca}$ and PO4 Release,” Journal of Dental Research 85, 722-727 (2006).

H. H. K. Xu, M. D. Weir, E. F. Burguera, and A. M. Fraser, "Injectable and Macroporous Calcium Phosphate Cement Scaffold,” Biomaterials 27, 4279-4287 (2006).

\section{Combinatorial Methods for Tissue Engineering}

Y. Mei, T. Wu, C. Xu, K. J. Langenbach, J. T. Elliott, B. D. Vogt, K. L. Beers, E. J. Amis, and N. R. Washburn, "Tuning Cell Adhesion on Gradient Poly(2-Hydroxyethyl Methacrylate)-Grafted Surfaces," Langmuir 21, 12309-12314 (2005).

C. G. Simon, N. Eidelman, S. B. Kennedy, A. Sehgal, C. A. Khatri, and N. R. Washburn, "Combinatorial Screening of Cell Proliferation on Poly(D,L-Lactic Acid)/Poly(D,L-Lactic Acid) Blends,” Biomaterials 26, 6906-6915 (2005).

Y. Mei, A. Kumar, W. Gao, R. Gross, S. B. Kennedy, N. R. Washburn, E. J. Amis, and J. T. Elliott, "Biocompatibility of Sorbitol-Containing Polyesters: Synthesis, Surface Analysis, and Cell Response in Vitro," Polymer Biocatalysis and Biomaterials 900, 343-353 (2005).

L. O. Bailey, S. Lipplatt, F. S. Biancanello, S. D. Ridder, and N. R. Washburn, "The Quantification of Cellular Viability and Inflammatory Response to Stainless Steel Alloys,” Biomaterials 26, 5296-5302 (2005).

C. G. Simon, J. M. Antonucci, D. W. Liu, and D. Skptic, "In Vitro Cytotoxicity of Amorphous Calcium Phosphate Composites," Journal of Bioactive and Compatible Polymers 20, 279-295 (2005).

K. Loo, S. B. Kennedy, N. Eidelman, Y. Tai, M. Zharnikov, E. J. Amis, A. Ulman, and R. A. Gross, "Combinatorial Approach to Study Enzyme/Surface Interactions,” Langmuir 21, 5237-5241 (2005).

B. Hammouda, F. Horkay, and M. L. Becker, "Clustering and Solvation in Poly(Acrylic Acid) Polyelectrolyte Solutions,” Macromolecules 38, 2019-2021 (2005).
Y. M. An, J. W. Cooper, B. M. Balgley, and C. S. Lee, "Selective Enrichment and Ultrasensitive Identification of Trace Peptides in Proteome Analysis Using Transient Capillary Isotachophoresis/Zone Electrophoresis Coupled With Nano-ESI-MS," Electrophoresis 27, 3599-3608 (2006).

M. L. Becker, N. D. Gallant, and E. J. Amis, “A Universal Substrate for Bio-Functionalization,” Abstracts of Papers of the American Chemical Society 231 (2006).

M. L. Becker, M. J. Joralemon, and K. L. Wooley, "Nanoparticles Decorated for Development As Synthetic Vaccines," Abstracts of Papers of the American Chemical Society 231 (2006).

C.G. Simon Jr, “Combinatorial Polymer Scaffold Library," in PTEI Regenerate 2006 World Congress on Tissue Engineering and Regenerative Medicine, Pittsburgh, PA.

Y. Yang, M.L. Becker, J. Kohn, and J.C.G. Simon, "Polymeric Combinatorial Scaffold Libraries for Screening Cell-Biomaterial Interactions," in 2006 International Microelectronics and Packaging Society Conference Proceeding, Chicago, IL.

S. B. Kennedy, N. R. Washburn, C. G. Simon, and E. J. Amis, "Combinatorial Screen of the Effect of Surface Energy on Fibronectin-Mediated Osteoblast Adhesion, Spreading and Proliferation,” Biomaterials 27, 3817-3824 (2006).

Y. Mei, J. T. Elliott, J. R. Smith, K. J. Langenbach, T. $\mathrm{Wu}, \mathrm{C} . \mathrm{Xu}, \mathrm{K}$. L. Beers, E. J. Amis, and L. Henderson, "Gradient Substrate Assembly for Quantifying Cellular Response to Biomaterials," Journal of Biomedical Materials Research Part A 79A, 974-988 (2006).

L. O. Bailey, M. L. Becker, J. S. Stephens, N. D. Gallant, C. M. Mahoney, N. R. Washburn, A. Rege, J. Kohn, and E. J. Amis, "Cellular Response to PhaseSeparated Blends of Tyrosine-Derived Polycarbonates," Journal of Biomedical Materials Research Part A 76A, 491-502 (2006).

L. O. Bailey, M. D. Weir, and N. R. Washburn, "Quantification of Macrophage Viability and Inflammatory Response to Dental Bonding Resins," Journal of Bioactive and Compatible Polymers 21, 185-206 (2006). 


\section{Combinatorial Methods (also see Combinatorial Methods for Tissue Engineering)}

\section{NIST Combinatorial Methods Center}

C.M. Stafford, K. E. Roskov, T. H. Epps, and M. J. Fasolka, "Generating Thickness Gradients of Thin Polymer Films Via Flow Coating," Review of Scientific Instruments 77, 023908 (2006)

M.J. Fasolka, "Experimental Approaches From A "Forward Looking" Laboratory: Polymer And Coatings Research At The NIST Combinatorial Methods Center," in Proceedings of the 33rd International Waterborne, High-Solids, and Powder Coatings Symposium, New Orleans, LA (2006).

I. Takeuchi, J. Lauterbach, M. J. Fasolka, "Combinatorial materials synthesis,” Materials Today 8, 18-26, (2005).

\section{Polymer Formulations}

C. Xu, T. Wu, Y. Mei, C. M. Drain, J. D. Batteas, and K. L. Beers, "Synthesis and Characterization of Tapered Copolymer Brushes Via Surface-Initiated Atom Transfer Radical Copolymerization,” Langmuir 21, 11136-11140 (2005).

S. Rathgeber, T. Pakula, A. Wilk, K. Matyjaszewski, and K. L. Beers, "On the Shape of Bottle-Brush Macromolecules: Systematic Variation of Architectural Parameters,” Journal of Chemical Physics 122, 124904 (2005).

E. J. Amis, K. L. Beers, and S. D. Hudson, "High Throughput Approaches to Analysis of Complex Mixtures," Abstracts of Papers of the American Chemical Society 231 (2006).

S. E. Barnes, Z. T. Cygan, J. K. Yates, K. L. Beers, and E. J. Amis, "Raman Spectroscopic Monitoring of Droplet Polymerization in a Microfluidic Device," Analyst 131, 1027-1033 (2006).

I. Norman, W. H. Zhang, K. L. Beers, and E. J. Amis, "Microfluidic Light Scattering As a Tool to Study the Structure of Aqueous Polymer Solutions," Journal of Colloid and Interface Science 299, 580-588 (2006).

I. Norman, D. L. Ho, J. H. Lee, and A. Karim, "Spontaneous Formation of Vesicles of Diblock Copolymer EO6BO11 in Water: A SANS Study," Journal of Physical Chemistry B 110, 62-67 (2006).
S. Rathgeber, T. Pakula, A. Wilk, K. Matyjaszewski, H. I. Lee, and K. L. Beers, "Bottle-Brush Macromolecules in Solution: Comparison Between Results Obtained From Scattering Experiments and Computer Simulations," Polymer 47, 7318-7327 (2006).

$\mathrm{Xu}, \mathrm{T}$. Wu, and K. L. Beers, "Patterned Polymer Brushes From Surface-Initiated Polymerization Inside a Microchannel," Abstracts of Papers of the American Chemical Society 231, 308 (2006).

C. Xu, S. E. Barnes, T. Wu, D. A. Fischer, D. M. DeLongchamp, J. D. Batteas, and K. L. Beers, "Solution and Surface Composition Gradients Via Microfluidic Confinement: Fabrication of a Statistical-CopolymerBrush Composition Gradient,” Advanced Materials 18, 1427-1430 (2006).

C. Xu, T. Wu, C. M. Drain, J. D. Batteas, M. J. Fasolka, and K. L. Beers, "Effect of Block Length on Solvent Response of Block Copolymer Brushes: Combinatorial Study With Block Copolymer Brush Gradients," Macromolecules 39, 3359-3364 (2006).

C. Xu, T. Wu, J. D. Batteas, C. M. Drain, K. L. Beers, and M. J. Fasolka, "Surface-Grafted Block Copolymer Gradients: Effect of Block Length on Solvent Response,” Applied Surface Science 252, 2529-2534 (2006).

K.L. Beers, "Controlled Radical Polymerization In Confined Spaces," in 4th IUPAC Sponsored Symposium on Radical Polymerization: Kinetics and Mechanism, Il Ciocco, Italy. (2006)

C. Xu and K.L. Beers, "Gradient Libraries Of Surface-Grafted Polymers: Combinatorial Tools For Surface Functionality," Proceedings Of the Annual Technical Meeting of the Society of Plastics Engineers (ANTEC), Charlotte, NC, (2006).

\section{Mechanics of Complex Interfaces}

C. M. Stafford, B. D. Vogt, C. Harrison, D. Julthongpiput, and R. Huang, "Elastic Moduli of Ultrathin Amorphous Polymer Films," Macromolecules 39, 5095-5099 (2006).

E. A. Wilder, S. Guo, S. Lin-Gibson, M. J. Fasolka, and C. M. Stafford, "Measuring the Modulus of Soft Polymer Networks Via a Buckling-Based Metrology," Macromolecules 39, 4138-4143 (2006).

H. Huang, X. Hu, C. Xu, K.L. Beers, and C.M. Stafford, "Construction Of Combinatorial Libraries To Study Adhesion In A Multilens Measurement Platform," in Adhesion Society Annual Meeting - Extended Abstracts, 2006, Jacksonville, FL. 
R. Huang, C.M. Stafford, and B.D. Vogt, "Wrinkling of ultrathin polymer films," in MRS Proceedings, 2006, San Francisco, CA.

J.H. Kim, M.Y. Chiang, D. Kawaguchi, G. Royston, and C.M. Stafford, "Estimation Of The Interfacial Adhesion Strength In Compositional Libraries Of Epoxy Films," in Adhesion Society Annual Meeting - Extended Abstracts, 2006, Jacksonville, FL.

P.M. McGuiggan and J. Filliben, "High Throughput Measurement of Peel of A Pressure Sensitive Adhesive," in Pressure Sensitive Tape Council Proceedings, Las Vegas (2006).

C.M. Stafford, "Application Of Combinatorial Methods For The Testing of Adhesives," in MII Technical Conference \& Review, 2006, Virginia Tech, Blacksburg, VA.

C.M. Stafford, J.H. Kim, D. Kawaguchi, G. Royston, and M.Y. Chiang, "Probing The Interfacial Adhesion Strength In Compositional Libraries Of Epoxy Films," in MRS Proceedings, 2006, Boston, MA.

C.M. Stafford, H.W. Ro, R.L. Jones, M.J. Fasolka, and C.L. Soles, "High-Throughput Screening of Adhesion and Release in Nanoimprint Lithography," in Adhesion Society Annual Meeting - Extended Abstracts, 2006, Jacksonville, FL.

P. M. McGuiggan, A. Chiche, F. Phelan, M. Fasolka, J. Filliben, and D. J. Yarusso, High Throughput Measurement of Peel of A Pressure Sensitive Adhesive. Adhesives and Sealants Industry Magazine April 2006, 32-39 (2006).

C.M. Stafford and C. Harrison, "Elastic Moduli of Polymer Thin Films: A High-throughput Metrology", in Encyclopedia of Materials: Science and Technology, 2006 Online Update. Editors: Buschow, Flemings, Cahn, Veyssière, Kramer, and Mahajan (Elsevier, 2006).

\section{Nanotechnology}

\section{Thin Films}

L. S. Goldner, M. J. Fasolka, and S. N. Goldie, "Measurement of the Local Diattenuation and Retardance of Thin Polymer Films Using Near-Field Polarimetry," Applications of Scanned Probe Microscopy to Polymers 897, 65-84 (2005).

D. C. Hurley, A. Kopycinska-Muller, D. Julthongpiput, and M. J. Fasolka, "Influence of Surface Energy and Relative Humidity on AFM Nanomechanical Contact Stiffness,” Applied Surface Science 253, 1274-1281 (2006).
P. M. McGuiggan and J. S. Wallace, "Maximum Force Technique for the Measurement of the Surface Tension of a Small Droplet by AFM,” Journal of Adhesion 82, 997-1011 (2006).

K. A. Page, F. A. Landis, A. K. Phillips, and R. B. Moore, "SAXS Analysis of the Thermal Relaxation of Anisotropic Morphologies in Oriented Nafion Membranes," Macromolecules 39, 3939-3946 (2006).

R. Jones, S. Kim, A. Karim, R. M. Briber, and H. C. Kim, "Orientation of Block Copolymer Phases Within Confined Environments," Abstracts of Papers of the American Chemical Society 231 (2006).

K. M. Ashley, D. Raghavan, J. F. Douglas, and A. Karim, "Wetting-Dewetting Transition Line in Thin Polymer Films,” Langmuir 21, 9518-9523 (2005).

P. Achalla, J. McCormick, T. Hodge, C. Moreland, P. Esnault, A. Karim, and D. Raghavan, "Characterization of Elastomeric Blends by Atomic Force Microscopy,” Journal of Polymer Science Part B-Polymer Physics 44, 492-503 (2006).

S. K. Satija, A. Karim, and J. Duangrut, “Tuning Interface Potential for Block Copolymer Thin Film Ordering," Abstracts of Papers of the American Chemical Society 231 (2006).

C. Soles, "X-Ray Reflectivity As an Effective Interface Metrology for Nanotechnology," Abstracts of Papers of the American Chemical Society 231 (2006).

Karim, J. Douglas, K. Ashley, and D. Raghavan, "Dewetting Transition in Thin Polymer Films," Proceedings Of the Annual Technical Meeting of the Society of Plastics Engineers (ANTEC), 2006, Charlotte, NC.

\section{Composites}

S. Balakrishnan, P. R. Start, D. Raghavan, and S. D. Hudson, "The Influence of Clay and Elastomer Concentration on the Morphology and Fracture Energy of Preformed Acrylic Rubber Dispersed Clay Filled Epoxy Nanocomposites," Polymer 46, 11255-11262 (2005).

T. Kashiwagi, F. M. Du, J. F. Douglas, K. I. Winey, R. H. Harris, and J. R. Shields, "Nanoparticle Networks Reduce the Flammability of Polymer Nanocomposites," Nature Materials 4, 928-933 (2005).

J. Bur, Y. H. Lee, S. C. Roth, and P. R. Start, "Measuring the Extent of Exfoliation in Polymer/Clay Nanocomposites Using Real-Time Process Monitoring Methods," Polymer 46, 10908-10918 (2005). 
T. Koga, C. Li, Y. Sun, A. Brazin, M. H. Rafailovich, J. C. Sokolov, J. F. Douglas, and D. Mahajan, "Surface Modification of Polymeric Nanocomposite Thin Films Using Supercritical Carbon Dioxide," Topics in Catalysis 32, 257-262 (2005).

E. J. Garboczi, J. F. Douglas, and R. B. Bohn, “A Hybrid Finite Element-Analytical Method for Determining the Intrinsic Elastic Moduli of Particles Having Moderately Extended Shapes and a Wide Range of Elastic Properties,” Mechanics of Materials 38, 786-800 (2006).

J. H. Kim, G. A. Holmes, and C. R. Snyder, "A Methodology for Detecting Interfacial Debonding in Clay/Epoxy Nanocomposites," Journal of Adhesion Science and Technology 20, 1079-1089 (2006).

C. K. Moon, G. A. Holmes, and W. G. McDonough, "Study on the Interfacial Properties of Two-Dimensionally Arranged Glass Fiber/Epoxy Resin Model Composites,” Journal of Applied Polymer Science 99, 1541-1551 (2006).

\section{Nanotubes}

E. K. Hobbie, B. J. Bauer, J. Stephens, M. L. Becker, P. McGuiggan, S. D. Hudson, and H. Wang, "Colloidal Particles Coated and Stabilized by DNA-Wrapped Carbon Nanotubes,” Langmuir 21, 10284-10287 (2005).

B. J. Bauer, E. K. Hobbie, and M. L. Becker, "SmallAngle Neutron Scattering From Labeled Single-Wall Carbon Nanotubes," Macromolecules 39, 2637-2642 (2006).

J. A. Fagan, B. J. Landi, I. Mandelbaum, J. R. Simpson, V. Bajpai, B. J. Bauer, K. Migler, A. R. H. Walker, R. Raffaelle, and E. K. Hobbie, "Comparative Measures of Single-Wall Carbon Nanotube Dispersion," Journal of Physical Chemistry B 110, 23801-23805 (2006).

D. Fry, B. Langhorst, H. Wang, M. L. Becker, B. J. Bauer, E. A. Grulke, and E. K. Hobbie, "Rheo-Optical Studies of Carbon Nanotube Suspensions," Journal of Chemical Physics 124, 054703 (2006).

E. K. Hobbie, J. Obrzut, S. B. Kharchenko, and E. A. Grulke, "Charge Transport in Melt-Dispersed Carbon Nanotubes," Journal of Chemical Physics 125, 044712 (2006).

F.R. Phelan Jr. and B.J. Bauer, "Simulation of Rodlike Particles in Field-Flow Fractionation (FFF)," in Proceedings of the 2006 AICHE Annual Meeting, San Francisco, CA.

B.J. Bauer, V. Bajpai, J.A. Fagan, M.L. Becker, and K. Hobbie Erik, "Chromatographic Separation of Single Wall Carbon Nanotubes," in MRS, Spring 2006, San Francisco, CA.

\section{Organic Electronics}

D. M. DeLongehamp, B. D. Vogt, C. M. Brooks, K. Kano, J. Obrzut, C. A. Richter, O. A. Kirillov, and E. K. Lin, "Influence of a Water Rinse on the Structure and Properties of Poly(3,4-Ethylene Dioxythiophene): Poly(Styrene Sulfonate) Films,” Langmuir 21, 11480-11483 (2005).

D. M. DeLongchamp, B. M. Vogel, Y. Jung, M. C. Gurau, C. A. Richter, O. A. Kirillov, J. Obrzut, D. A. Fischer, S. Sambasivan, L. J. Richter, and E. K. Lin, "Variations in Semiconducting Polymer Microstructure and Hole Mobility With Spin-Coating Speed," Chemistry of Materials 17, 5610-5612 (2005).

A. R. Murphy, P. C. Chang, P. VanDyke, J. S. Liu, J. M. J. Frechet, V. Subramanian, D. M. DeLongchamp, S. Sambasivan, D. A. Fischer, and E. K. Lin, "SelfAssembly, Molecular Ordering, and Charge Mobility in Solution-Processed Ultrathin Oligothiophene Films," Chemistry of Materials 17, 6033-6041 (2005).

N. Tsutsumi, A. Ueyasu, W. Sakai, and C. K. Chiang, "Crystalline Structures and Ferroelectric Properties of Ultrathin Films of Vinylidene Fluoride and Trifluoroethylene Copolymer," Thin Solid Films 483, 340-345 (2005).

M. L. Chabinyc, F. Endicott, B. D. Vogt, D. M. DeLongchamp, E. K. Lin, Y. L. Wu, P. Liu, and B. S. Ong, "Effects of Humidity on Unencapsulated Poly(Thiophene) Thin-Film Transistors," Applied Physics Letters 88, 113514 (2006).

D. M. DeLongchamp, M. M. Ling, Y. Jung, D. A. Fischer, M. E. Roberts, E. K. Lin, and Z. N. Bao, "Thickness Dependence of Microstructure in Semiconducting Films of an Oligofluorene Derivative," Journal of the American Chemical Society 128, 16579-16586 (2006).

D. M. DeLongchamp, D. A. Fischer, B. M. Vogel, R. J. Kline, Y. Jung, M. Gurau, L. J. Richter, and E. K. Lin, "Near-Edge X-Ray Absorption Fine Structure Spectroscopy As a Characterization Tool for Organic Semiconductors," Abstracts of Papers of the American Chemical Society 231 (2006).

E. K. Lin, "Developing Metrology to Enable the Realization of Organic Electronics Devices," Abstracts of Papers of the American Chemical Society 231 (2006).

J. Obrzut, "Nonlinear Charge Transport in Semiconducting Polythiophene," Abstracts of Papers of the American Chemical Society 231 (2006). 
Z. D. Schultz, M. Gurau, D. M. DeLongchamp, B. M. Vogel, R. J. Kline, Y. Jung, E. K. Lin, and L. J. Richter, "Nonlinear Spectroscopic Characterization of Polymer Thin Films," Abstracts of Papers of the American Chemical Society 231 (2006).

D.M. DeLongchamp, E.K. Lin, and D.A. Fischer , "Organic Semiconductor Structure and Chemistry from Near-edge X-ray Absorption Fine Structure (NEXAFS) Spectroscopy," in Organic Thin Film Transistors, (Taylor \& Francis, 2006).

R. Hamilton, C. Bailey, W. Duffy, M. Heeney, M. Shkunov, D. Sparrowe, S. Tierney, I. McCulloch, R.J. Kline, D.M. DeLongchamp, and M. Chabinyc, "The influence of molecular weight on the microstructure and thin film transistor characteristics of pBTTT polymers," in Proceedings of the SPIE, Vol. 6336, San Diego, CA (2006).

R.J. Kline and M.D. McGehee, "Charge Transport And Morphology In Conjugated Polymers" in Handbook of Organic Electronics and Photonics edited by H.S. Nalwa (American Scientific Publishers, 2006).

\section{Polymers for Next Generation Lithography}

V. M. Prabhu, B. D. Vogt, W. L. Wu, J. F. Douglas, E. K. Lin, S. K. Satija, D. L. Goldfarb, and H. Ito, "Direct Measurement of the Counterion Distribution Within Swollen Polyelectrolyte Films," Langmuir 21, 6647-6651 (2005).

B. D. Vogt, R. A. Pai, H. J. Lee, R. C. Hedden, C. L. Soles, W. L. Wu, E. K. Lin, B. J. Bauer, and J. J. Watkins, "Characterization of Ordered Mesoporous Silica Films Using Small-Angle Neutron Scattering and X-Ray Porosimetry,” Chemistry of Materials 17, 1398-1408 (2005).

X. F. Hua, M. S. Kuo, G. S. Oehrlein, P. Lazzeri, E. Iacob, M. Anderle, C. K. Inoki, T. S. Kuan, P. Jiang, and W. L. Wu, "Damage of Ultralow k Materials During Photoresist Mask Stripping Process," Journal of Vacuum Science \& Technology B 24, 1238-1247 (2006).

S. H. Kang, V. M. Prabhu, B. D. Vogt, E. K. Lin, W. L. Wu, and K. Turnquest, "Effect of Copolymer Composition on Acid-Catalyzed Deprotection Reaction Kinetics in Model Photoresists," Polymer 47, 6293-6302 (2006).

E. K. Lin, V. M. Prabhu, B. D. Vogt, S. H. Kang, A. Rao, S. K. Satija, W. L. Wu, and K. Turnquest, "Measurements Towards the Materials Sources of Line-Edge Roughness in Chemically Amplified Photoresists," Abstracts of Papers of the American Chemical Society 231 (2006).
V. M. Prabhu, S. Sambasivan, D. Fischer, L. K. Sundberg, and R. D. Allen, "Quantitative Depth Profiling of Photoacid Generators in Photoresist Materials by NearEdge X-Ray Absorption Fine Structure Spectroscopy,” Applied Surface Science 253, 1010-1014 (2006).

Rao, S. H. Kang, B. D. Vogt, V. M. Prabhu, E. K. Lin, W. L. Wu, and M. Muthukumar, "Effect of Deprotection Extent on Swelling and Dissolution Regimes of Thin Polymer Films," Langmuir 22, 10009-10015 (2006).

B. D. Vogt, S. Kang, V. M. Prabhu, E. K. Lin, S. K. Satija, K. Turnquest, and W. L. Wu, "Measurements of the Reaction-Diffusion Front of Model Chemically Amplified Photoresists With Varying Photoacid Size," Macromolecules 39, 8311-8317 (2006).

S. Kang, V.M. Prabhu, B.D. Vogt, E.K. Lin, W. Wu, and K. Turnquest, "Copolymer Fraction Effect On Acid Catalyzed Deprotection Reaction Kinetics In Model 193 Nm Photoresists," in Proceedings of the SPIE Advances in Resist Materials and Processing XXIII, San Jose, CA (2006).

S. Kang, V.M. Prabhu, B.D. Vogt, E.K. Lin, W. Wu, and K. Turnquest, "Effect of copolymer composition on acid catalyzed deprotection reaction kinetics in model photoresists," in Proceedings of the SPIE Advances in Resist Materials and Processing XXIII, San Jose, CA (2006).

K.A. Lavery, K.-W. Choi, B.D. Vogt, V.M. Prabhu, E.K. Lin, W. Wu, S. Satija, M.J. Leeson, H.B. Cao, G. Thompson, H. Ding, and D.S. Fryer, "Fundamentals Of The Reaction-Diffusion Process In Model Euv Photoresists," in Proceedings of the SPIE Advances in Resist Materials and Processing XXIII, San Jose, CA (2006).

Rao, S. Kang, B.D. Vogt, V.M. Prabhu, E.K. Lin, W. Wu, K. Turnquest, and W.D. Hinsberg, "Dissolution Fundamentals Of 193nm Methacrylate Based Photoresists," in Proceedings of the SPIE Advances in Resist Materials and Processing XXIII, San Jose, CA (2006).

B.D. Vogt, S. Kang, V.M. Prabhu, A. Rao, E.K. Lin, S.K. Satija, K. Turnquest, and W. Wu, "The Deprotection Reaction Front Profile For Model 193 Nm Methacrylate-Based Chemically Amplified Photoresists," in Proceedings of the SPIE Advances in Resist Materials and Processing XXIII, San Jose, CA (2006).

\section{Metrology for Nanoimprint Lithography}

R. L. Jones, T. J. Hu, C. L. Soles, E. K. Lin, R. M. Reano, and S. M. Casa, "Real-Time Shape Evolution of Nanoimprinted Polymer Structures During Thermal Annealing," Nano Letters 6, 1723-1728 (2006). 
C. Soles, H. W. Ro, Y. F. Ding, R. L. Jones, D. Hines, E. K. Lin, and A. Karim, "Stability and Processing Induced Residual Stress in Imprinted Polymer Nanostructures," Abstracts of Papers of the American Chemical Society 231 (2006).

R. L. Jones, C. L. Soles, E. K. Lin, W. Hu, R. M. Reano, S. W. Pang, S. J. Weigand, D. T. Keane, and J. P. Quintana, "Pattern Fidelity in Nanoimprinted Films Using Critical Dimension Small Angle X-Ray Scattering," Journal of Microlithography Microfabrication and Microsystems 5, 013001 (2006).

H.-J. Lee, C.L. Soles, H.W. Ro, D.R. Hines, R.L. Jones, E.K. Lin, A. Karim, and W. Wu, "Characterizing Nanoimprint Pattern Cross Section And Fidelity From X-Ray Reflectivity," in Proceedings of the SPIE Microlithography, San Jose, CA (2006).

H. J. Lee, H. W. Ro, C. L. Soles, R. L. Jones, E. K. Lin, W. L. Wu, and D. R. Hines, "Effect of Initial Resist Thickness on Residual Layer Thickness of Nanoimprinted Structures," Journal of Vacuum Science \& Technology B 23, 3023-3027 (2005).

H. J. Lee, C. L. Soles, H. W. Ro, R. L. Jones, E. K. Lin, W. L. Wu, and D. R. Hines, "Nanoimprint Pattern Transfer Quality From Specular X-Ray Reflectivity," Applied Physics Letters 87, 263111 (2005).

\section{Directed Assembly and Nanomanufacturing}

K. Van Workum and J. F. Douglas, "Schematic Models of Molecular Self-Organization," Macromolecular Symposia 227, 1-15 (2005).

J. F. Douglas and K. Van Workum, "Symmetry, Equivalence and Molecular Self-Organization," Abstracts of Papers of the American Chemical Society 229, U724-U725 (2005).

K. Van Workum and J. F. Douglas, "Equilibrium Polymerization in the Stockmayer Fluid As a Model of Supermolecular Self-Organization," Physical Review E 71, 031502 (2005).

S. D. Hudson, J. T. Cabral, W. J. Goodrum, K. L. Beers, and E. J. Amis, "Microfluidic Interfacial Tensiometry,” Applied Physics Letters 87, 081905 (2005).

J. T. Cabral and S. D. Hudson, "Microfluidic Approach for Rapid Multicomponent Interfacial Tensiometry," Lab on A Chip 6, 427-436 (2006).

J. A. Pathak and S. D. Hudson, "Rheo-Optics of Equilibrium Polymer Solutions: Wormlike Micelles in Elongational Flow in a Microfluidic Cross-Slot," Macromolecules 39, 8782-8792 (2006).
P. R. Start, S. D. Hudson, E. K. Hobble, and K. B. Migler, "Breakup of Carbon Nanotube Flocs in Microfluidic Traps," Journal of Colloid and Interface Science 297, 631-636 (2006).

P. A. Stone, S. D. Hudson, P. Dalhaimer, D. E. Discher, E. J. Amis, and K. B. Migler, "Dynamics of Wormlike Micelles in Elongational Flows," Macromolecules 39, 7144-7148 (2006).

K. Van Workum and J. F. Douglas, "Symmetry, Equivalence, and Molecular Self-Assembly," Physical Review E 73, 031502 (2006).

J. Stambaugh, K. Van Workum, J. F. Douglas, and W. Losert, "Polymerization Transitions in Two-Dimensional Systems of Dipolar Spheres,” Physical Review E 72, 031301 (2005).

J. J. Benkoski, R. L. Jones, J. F. Douglas, and A. Karim, "Competition of Forces Driving Assembly in Nanoscale Building Blocks," Abstracts of Papers of the American Chemical Society 231 (2006).

J. J. Benkoski, H. Hu, and A. Karim, "Generation of Hierarchical Topologies From Photocrosslinkable, Particle-Stabilized Emulsions," Macromolecular Rapid Communications 27, 1212-1216 (2006).

\section{Dimensional Metrology of Nanoscale Patterns}

M. S. Silverstein, M. Shach-Caplan, B. J. Bauer, R. C. Hedden, H. J. Lee, and B. G. Landes, "Nanopore Formation in a Polyphenylene Low-k Dielectric," Macromolecules 38, 4301-4310 (2005).

S. Knight, R. Dixson, R. L. Jones, E. K. Lin, N. G. Orji, R. Silver, J. S. Villarrubia, A. E. Vladar, and W. L. Wu, "Advanced Metrology Needs for Nanoelectronics Lithography,” Comptes Rendus Physique 7, 931-941 (2006).

H. J. Lee, C. L. Soles, D. W. Liu, B. J. Bauer, E. K. Lin, and W. L. Wu, "Structural Characteristics of Methylsilsesquioxane Based Porous Low-k Thin Films Fabricated With Increasing Cross-Linked Particle Porogen Loading," Journal of Applied Physics 100, 064104 (2006).

E. K. Lin, "Advancing Nanofabrication With Small Angle Scattering and Reflectivity Measurements," $A b$ stracts of Papers of the American Chemical Society 231 (2006).

M. S. Silverstein, B. J. Bauer, R. C. Hedden, H. J. Lee, and B. G. Landes, "SANS and XRR Porosimetry of a Polyphenylene Low-k Dielectric," Macromolecules 39, 2998-3006 (2006). 
R.L. Jones, C. Wang, E.K. Lin, W. Wu, K. Choi, B.J. Rice, G.M. Thompson, S.J. Weigand, and D.T. Keane, "Characterization Of Line Edge Roughness Using CDSAXS," in Proceedings of the SPIE Microlithography, San Jose, CA (2006).

V.R. Tirumala, B.D. Vogt, E.K. Lin, and J.J. Watkins, "Influence of Strongly Interacting Additives on the Long-range Order in Semi-crystalline Block Copolymer Templates," in American Chemical Society Polymer

Preprints 2006, San Francisco, CA (2006). 


\section{Polymers Division}

\section{Chief}

Eric J. Amis

Phone: 301-975-6762

E-mail: eric.amis@nist.gov

\section{Deputy Chief}

Chad R. Snyder

Phone: 301-975-4526

E-mail: chad.snyder@nist.gov

\section{NIST Fellow}

\section{Wen-li Wu}

Phone: 301-975-6839

E-mail: wen-li.wu@nist.gov

\section{Group Leaders}

Characterization and Measurement

Chad R. Snyder

Electronics Materials

Eric K. Lin

Phone: 301-975-6743

E-mail: eric.lin@nist.gov

\section{Biomaterials}

Marcus T. Cicerone

Phone: 301-975-8104

E-mail: marcus.cicerone@nist.gov

Multiphase Materials

Alamgir Karim

Phone: 301-975-6588

E-mail: alamgir.karim@nist.gov

Processing Characterization

Kalman Migler

Phone: 301-975-4876

E-mail: kalman.migler@nist.gov

Multivariant Measurement Methods

Michael Fasolka

Phone: 301-975-8526

E-mail: michael.fasolka@nist.gov 


\section{Research Staff}

\section{Alvine, Kyle*}

kyle.alvine@nist.gov

X-ray reflectivity \& GISAXS nanostructures

Nanoparticle self-assembly

Small angle $\mathrm{x}$-ray scattering

Nanoimprint lithography

Surface tension of nanostructures

\section{Amis, Eric J.}

eric.amis@nist.gov

Neutron, x-ray and light scattering

Polyelectrolytes

Viscoelastic behavior of polymers

Dendrimers and dendritic polymers

Functional biomaterials

Combinatorial methods

High-throughput experimentation

Antonucci, Joseph M.

joseph.antonucci@nist.gov

Synthetic polymer chemistry

Dental composites, cements and adhesion

Initiator systems

Interfacial coupling agents

Remineralizing polymer systems

Nanocomposites

Barnes, Susan E. +

susan.barnes@nist.gov

Combinatorial and high-throughput methods

Vibrational spectroscopy of polymers

Microfluidics technology

Fluorescence spectroscopy

On-line monitoring of polymer melts/extrusion

Bauer, Barry J.

barry.bauer@nist.gov

Polymer synthesis

Polymer chromatography

MALDI mass spectroscopy

Thermal characterization

Neutron, $x$-ray and light scattering

Dendrimers, metallic ions nanocluster

Porous low-k thin film characterization

Carbon nanotubes

\section{Becker, Matthew L.}

matt.becker@nist.gov

Polymer synthesis

Block copolymers

Peptide synthesis

Phage display

Combinatorial methods

Polymerase Chain Reaction
Beers, Kathryn L.

kathryn.beers@nist.gov

Combinatorial and high-throughput methods

Polymer formulations

Microfluidics technology

Polymer synthesis

Controlled/living polymerizations

Berry, Brian+

brian.berry@nist.gov

Inorganic nanoparticle synthesis

Thin film zone-annealing methods

Defects in nanostructured films

Block copolymer films

Combinatorial surface modification

Neutron reflectivity and small angle scattering

Blair, William R.

william.blair@nist.gov

Polymer analysis by size exclusion chromatography

Mass spectrometry of polymers

High temperature viscometry

Rayleigh light scattering

Extrusion plastometry

Bowen, Rafael L.*

rafael.bowen@nist.gov

Adhesion

Dental composites

Novel monomer synthesis

Bur, Anthony J.

anthony.bur@nist.gov

Dielectric properties of polymers

Fluorescence and optical monitoring of polymer processing

Piezoelectric, pyroelectric polymers

Viscoelastic properties of polymers

\section{Carey, Clifton M.*}

clifton.carey@nist.gov

Dental plaque

Microanalytical analysis techniques

Fluoride efficacy for dental health

De- and re-mineralization

Phosphate chemistry

Ion-selective electrodes

Toothpaste abrasion \& erosion

Cherng, Maria*

maria.cherng@nist.gov

Calcium phosphate biomaterials 


\section{Chastek, Thomas Q.} thomas.chastek@nist.gov

Combinatorial and high-throughput methods

Optics and fiber optics

Optical microscopy

Liquid chromatography

Microfluidics

Small angle $\mathrm{x}$-ray scattering

\section{Chastek, Thuy T.+}

thuy.chastek@nist.gov

Synthetic hybrid nanocomposites

Solid state synthesis

Electron microscopy

Rheology of nanocomposites and gels

Wide and small angle $\mathrm{x}$-ray diffraction

Self-assembly \& lithiography

Microfluidics

\section{Chiang, Chwan K.}

c.chiang@nist.gov

Electroluminescent polymers

Residual stress

Impedance spectroscopy

\section{Chiang, Martin Y.}

\section{martin.chiang@nist.gov}

Computational mechanics (finite element analysis)

Strength of materials, fracture mechanics

Engineering mechanics of polymer-based materials

Bi-material interface

Image quantitation

\section{Choi, Kwang-Woo+}

kwang-woo.choi@nist.gov

Polymers for lithography

Critical dimension small angle $\mathrm{x}$-ray scattering

(CD-SAXS)

Extreme ultraviolet (EUV) lithography

\section{Chow, Laurence C.*}

laurence.chow@nist.gov

Calcium phosphate compounds and biomaterials

Tooth demineralization and remineralization

Dental and biomedical cements

Solution chemistry

Dental caries prevention

\section{Chung, Jun Young+}

junyoung.chung@nist.gov

Combinatorial and high-throughput methods

Mechanics of complex interfaces

Stress relaxation and aging in thin polymer films Adhesion and fracture at soft interfaces
Cicerone, Marcus T.

marcus.cicerone@nist.gov

Protein stabilization

Glass transition theory

Optical coherence microscopy

Tissue engineering scaffolds

Confocal microscopy

Spectroscopic imaging

Cipriano, Bani H.+

Polymer rheology

Cooper, James A.

james.cooper@nist.gov

Tissue engineering

Polymer scaffolds

Cell biology

Optical microscopy

DeLongchamp, Dean M.

dean.delongchamp@nist.gov

Organic electronics

Polymer thin films

Polyelectrolytes

Near-edge x-ray absorption fine structure spectroscopy (NEXAFS)

Film electrochemistry

Dickens, Sabine*

sabine.dickens@nist.gov

Dental composites

Dental adhesives

Transmission electron microscopy

Remineralizing resin-based calcium phosphate composites and cements

Di Marzio, Edmund A.+ edmund.dimarzio@nist.gov

Statistical mechanics of polymers

Phase transitions

Glasses

Polymers at interfaces

Ding, Yifu+

yifu.ding@nist.gov

Polymer dynamics

Glass transition and glass properties

Inelastic light and neutron scattering

Nanoimprint lithography

Douglas, Jack F.

jack.douglas@nist.gov

Theory on polymer solutions, blends, and nanoparticle filled polymer materials

Physics of polymers films

Dynamics and thermodynamics of self-assembly

Crystallization of polymers

Defects and ordering in block copolymer films

Nanoparticle characterization and interactions 
Dunkers, Joy P. joy.dunkers@nist.gov

Optical coherence microscopy

Image analysis

Fiber optic spectroscopy

Infrared microspectroscopy of polymers

Confocal fluorescence microscopy

Duppins, Gretchen E.*

gretchen.duppins@nist.gov

Editorial Coordinator

\section{Dutta Roy, Tithi}

tithi.duttaroy@nist.gov

Reference scaffolds for tissue engineering

Cellular response to biomaterials

Eichmiller, Frederick C.*

frederick.eichmiller@nist.gov

Clinical dentistry

Composites

Dentin adhesives

Polymerization shrinkage

Eidelman, Naomi B.*

naomi.eidelman@nist.gov

FTIR microspectroscopy

Characterization of dental tissues and materials

Composition of combinatorial polymer blends

Appplication of temperature and UV gradients to polymers

Epps, Thomas H., III

thomas.epps@nist.gov

Combinatorial and high-throughput methods

Block copolymers

Self-assembled structures

Surface energy patterning and control

Surfaces and interfaces

Scanning probe microscopy

Fagan, Jeffrey A.

jeffrey.fagan@nist.gov

Dielectrophoretic separations

Colloidal solutions

Electrooptical effects

Carbon nanotubes

Fasolka, Michael J.

michael.fasolka@nist.gov

Combinatorial and high-throughput methods

Combinatorial and high-throughput methods

NIST Combinatorial Methods Center (NCMC)

Self-assembled structures

Surface energy patterning and control

Surfaces and interfaces

Scanning probe microscopy
Flaim, Glenn M.*

glenn.flaim@nist.gov

Fabricating dental composites

Floyd, Cynthia J. E.*

cynthia.floyd@nist.gov

Dental composites

Nuclear magnetic resonance (NMR)

Flynn, Kathleen M.

kathleen.flynn@nist.gov

Melt flow rate measurements

Size exclusion chromatography

Mass spectrometry of polymers

Fowler, Bruce O.+

bruce.fowler@nist.gov

Infrared and Raman spectroscopy

Structure of calcium phosphates, bones, and teeth

Composites

Frukhtbeyn, Stanislav*

stan.frukhtbeyn@nist.gov

Calcium phosphate compounds and biomaterials

Topical dental fluorides

Gallant, Nathan D.

nathan.gallant@nist.gov

Cell adhesion to biomaterials

Combinatorial screening of bioactive gradients

Genson, Kirsten L.

kirsten.genson@nist.gov

Highly branched polymers

Surface initiated polymerization

Surface functionalization

Atomic force microscopy

Gradient combinatorial methods

George, Laurie A.*

laurie.george@nist.gov

Network Administrator

Giuseppetti, Anthony A.*

anthony.giuseppetti@nist.gov

Casting of dental alloys

Scanning electron microcopy

Dental materials testing

Guo, Shu+

shu.guo@nist.gov

Solid mechanics

Mechanical properties of thin films

Combinatorial and high-throughput methods

Polymer thin films

Surfaces and interfaces 


\section{Guttman, Charles M.}

charles.guttman@nist.gov

Solution properties of polymers

Size exclusion chromatography

Mass spectrometry of polymers

Han, Charles C.+

charles.han@nist.gov

Phase behavior of polymer blends

Phase separation kinetics of polymer blends

Polymer characterization and diffusion

Shear mixing/demixing and morphology control of polymer blends

Static, time resolved, and quasi-elastic scattering

Henderson, Lori A.

lori.henderson@nist.gov

Structure-property relationships of biomaterials

Structure-function of tissues

Molecular engineering of DNA and proteins

Cellular physiology and assays

Molecular biology screening

Polymer synthesis and characterization

Ho, Derek H+

dereklho@nist.gov

Small angle neutron scattering

Small angle X-ray scattering

Clay-polymer nanocomposites

Hobbie, Erik K.

erik.hobbie@nist.gov

Light scattering and optical microscopy

Dynamics of complex fluids

Shear-induced structures in polymer blends and solutions

Carbon nanotubes suspensions and melts

\section{Hodkinson, Christine S.*}

\section{christine.hodkinson@nist.gov}

Manager, Administrative Services

Holmes, Gale A.

gale.homes@nist.gov

Composite interface science

Chemical-structure-mechanical property

relationships for:

Polymer chemistry

Mass spectroscopy

Nanocomposites

Ballistic resistance

\section{Hu, Xuesong+}

xuesong.hu@nist.gov

Combinatorial Informatics

Instrument automation

Adhesion and mechanical properties
Huang, Heqing +

heqing.huang@nist.gov

Combinatorial and high-throughput methods

Adhesion and mechanical properties

Surface initiated polymerization

Polymer brushes

\section{Hudson, Steven D.}

steven.hudson@nist.gov

Electron and optical microscopy

Microfluidics

Nanoparticle characterization and assembly

Self-assembly

Bio- and interfacial dynamics

Iida, Kazunori+

kazunori.iida@nist.gov

Combinatorial and high-throughput methods

Microfluidics and microreactors

Controlled radical polymerization

Living radical polymerization

Johnson, Jerainne

jerainne.johnson@nist.gov

Spectroscopy, fluorescence upconversion and time resolved fluorescence

Bench top method for evaluating materials for protein storage

Jones, Ronald L.

ronald.jones@nist.gov

Neutron and x-ray scattering

Defects in nanomaterials

Neutron reflectivity

3-D tomography

Polymer surfaces and thin films

Polymer phase transitions and computer simulation

Julthongpiput, Duangrut + duangrut.julthongpiput@nist.gov

Combinatorial and high-throughput methods Surface energy patterning and control

Scanning probe microscopy

Jung, Youngsuk+ +

youngsuk.jung@nist.gov

Organic electronics

Polymer thin films and interfaces

Kang, Shuhui +

skang@nist.gov

Fourier transform infrared spectroscopy (FTIR)

Raman spectroscopy

Polymers for lithography

Polymer thin films 
Karim, Alamgir

alamgir.karim@nist.gov

Combinatorial and high-throughput methods

Patterning of thin-polymer blend films on

inhomogenous surfaces

Neutron \& x-ray reflection and scattering

AFM and optical microscopy

Nanofilled polymer films

Nanostructured materials

Metrology for nanoscale manufacturing

Kee, Tak+

tak.kee@nist.gov

Ultrafast spectroscopy

Coherent anti-Stokes Raman scattering (CARS) microscopy

Tissue engineering scaffolds

Confocal microscopy

Khoury, Freddy A.

freddy.khoury@nist.gov

Crystallization, structure and morphology of polymers

Analytical electron microscopy of polymers

Wide-angle and small-angle x-ray diffraction

Structure and mechanical property relationships

Kim, Jae Hyun+

jaehyun@nist.gov

Fiber/matrix interface

Polymer adhesion and mechanical properties

Polymer composites

Optical coherence microscopy

Kim, Sangcheol

sangcheol.kim@nist.gov

Ordering and defects in thin nanostrucutered materials

Combinatorial and high-throughput methods

AFM and optical microscopy

$\mathrm{X}$-ray reflectivity

Kipper, Matthew +

matthew.kipper@nist.gov

Hemotactic, chemotactic response to biomaterials

Craniofacial tissue engineering

Cell migration

\section{Kline, R. Joseph}

rjkline@nist.gov

Organic electronics

Grazing incidence $\mathrm{x}$-ray diffraction

Polymer thin films morphology

AFM characterization
Lacerda, Silvia+

silvia.lacerda@nist.gov

Quantum dots synthesis and surface functionalization

Quantum dot-based biosensors

Cell-penetrating proteins and peptides for delivery of nanoparticles in living cells

Interaction of nanoparticles with animal tissues

Transmission Electron Microscopy, 3D tomography

Defects in block copolymer films

Assembling of particles in nanoporous membranes

Landis, Forrest A.+

forrest.landis@nist.gov

Crystallization and melting of miscible polymer blends

Ionomers

Optical coherence microscopy

Tissue engineered scaffolds

Static small angle laser light scattering

\section{Laumeier, Carol E.}

carol.laumeier@nist.gov

NIST Combinatorial Methods Center

Outreach Coordinator

Lavery, Kristopher A.

klavery@nist.gov

Polymer thin films and interfaces

Neutron and X-ray reflectometry

Off-specular reflectivity

Photoresist materials

Diblock copolymer thin films

Lee, Hae-Jeong+

hae-jeong.lee@nist.gov

X-ray reflectivity

Small-angle neutron scattering

Nanoimprint lithography

Structural characterization of low dielectric constant thin films

Porosimetry of porous thin films

Metrology for characterizing physical structures of nanopatterns

Lin, Eric K.

eric.lin@nist.gov

Polymer thin films and interfaces

Polymer photoresists for lithography

Organic electronics

Nanoimprint lithography

Small angle X-ray and neutron scattering

Statistical mechanics

$\mathrm{X}$-ray and neutron reflectivity 
Lin, Nancy J. nancy.lin@nist.gov

Combinatorial screening of scaffolds

Cellular response to materials

\section{Lin-Gibson, Sheng}

sheng.lin-gibson@nist.gov

Rheology of gels and nanocomposites

Mass spectrometry of synthetic polymers

Polymer synthesis and modification

Structure and dynamics of nanocomposite polymeric materials

Tissue engineering hydrogels

Liu, Da-Wei

da-wei.liu@nist.gov

Polymer synthesis

Thermal gravimetric analysis

Differential scanning calorimetry

Gel permeation chromatography

Infrared spectroscopy

Nuclear magnetic resonance

Lucas, Leah A.+

leah.lucas@nist.gov

Organic electronics

Combinatorial methods

Surface functionalization

\section{Markovic, Milenko*}

milenko.markovic@nist.gov

Calcium phosphate chemistry

Biomineralization (normal and pathological)

Crystal growth and dissolution kinetics

Heterogeneous equilibria

McDonough, Walter G.

walter.mcdonough@nist.gov

Processing and cure monitoring polymer

composites

Failure and fracture of polymers

Polymer composite interfaces

Dental materials

McGuiggan, Patricia

patricia.mcguiggan@nist.gov

Atomic force microscopy

Viscoelastic properties

Surface force measurements

\section{Meillon, Mathurin+}

mathurin.meillon@nist.gov

Polymer rheology

Characterization of processing aids
Migler, Kalman

kalman.migler@nist.gov

Rheology

Carbon nanotubes

Liquid crystals

Polymer blends

Polymer slippage

Sharkskin and extrusion

Obrzut, Jan

\section{jan.obrzut@nist.gov}

Dielectric relaxation spectroscopy

Electronic properties of polymers and composites

Electronic packaging

Microwave and optical waveguides

Photoelectron spectroscopy (x-ray and UV)

Reliability, stress testing

Okerberg, Brian C.

brian.okerberg@nist.gov

Polymer crystallization and morphology

Thin films and polymer blends

Hot-stage atomic force microscopy

Nanoimprint lithography

Optical and electron microscopy

Page, Kirt A.

kirt.page@nist.gov

X-ray scattering

Neutron scattering

Polymer structure and dynamics

Polymer brushes

Polymer Ionomers

Membranes

Combinatorial methods

Pakstis, Lisa

lisa.pakstis@nist.gov

Self-assembly of amphiphilic diblock copolypeptides

Biomaterial-cell interactions

Physical characterization of polymer/biomaterial assemblies

Microscopy, scattering, rheology, spectroscopy, cell culture

Park, Jung J.+

jungjin.park@nist.gov

Microfluidics

Bioadhesion properties of nanoparticles

Biopolymer interactions

Parry, Edward E.*

edward.parry@nist.gov

Dental appliance and crown and bridges fabrication

Machine shop applications 
Pathak, Jai A.+

jai.pathak@nist.gov

Rheology and linear viscoelasticity

Polymer dynamics and complex fluids

Microfluidics

Patton, Derek L.

derek.patton@nist.gov

Combinatorial and high-throughput methods

Polymer brushes

Controlled radical polymerization

Surface functionalization

Atomic force microscopy

X-ray Photoelectron Spectroscopy

Phelan, Jr., Frederick R.

frederick.phelan@nist.gov

Composites processing

Microfluidics

Viscoelastic flow modeling

Chaotic mixing

Flow in porous media

Lattice Boltzmann methods

Prabhu, Vivek M.

vivek.prabhu@nist.gov

Small-angle neutron scattering

Polyelectrolytes

Polymers for lithography

Fluorescence correlation spectroscopy

Polymer thin films

$\mathrm{X}$-ray and neutron reflectivity

\section{Psurek Tatiana+}

tatiana.psurek@nist.gov

Dielectric spectroscopy

Organic Electronics

Polymer thin films

Quinn, Janet*

janet.quinn@nist.gov

Fractography

Dental materials and material properties

Composites

\section{Richards, Nicola*}

nicola.richards@nist.gov

Dental restorative materials

Polymer matrix composites

\section{Rao, Ashwin B. +}

ashwin@nist.gov

Polymer adsorption

Thin films gels

Fluorescence microscopy

Interfacial rheology
Ro, Hyun Wook+

hyun.ro@nist.gov

Nanoimprint lithography

Low-k dielectric thin films

$\mathrm{X}$-ray reflectivity

Sambasivan, Sharadha+

sharadha@bnl.gov

Near-edge X-ray absorption fine structure spectroscopy (NEXAFS)

Polymers for lithography

Polymer relaxation and tribology

Self assembled monolayer orientation

Catalyst surface and bulk characterization

Schumacher, Gary E.*

gary.schumacher@nist.gov

Clinical dentistry

Composites

Dentin adhesives

Simon, Carl G., Jr.

carl.simon@nist.gov

Biocompatibility

Cytotoxicity

Signaling in human platelets

Bone marrow cell lineage/trafficking

Combinatorial methods

Skrtic, Drago*

drago.skrtic@nist.gov

Bioactive amorphous calcium phosphate-based dental materials

Smith, Jack R.

jack.smith@nist.gov

Surface science

Computational modeling

Biomaterials characterization

Snyder, Chad R.

chad.snyder@nist.gov

Dielectric relaxation spectroscopy

Thermodynamics and kinetics of polymer crystallization

Calorimetry

Thermal properties at the nanoscale

Ballistic resistance of polymers

Soles, Christopher L.

csoles@nist.gov

Polymer dynamics

Inelastic neutron scattering

Low-k dielectric thin films

$\mathrm{X}$-ray and neutron reflectivity

Polymer thin films and lithography

Ion beam scattering

Nanoimprint lithography 
Stafford, Christopher M. chris.stafford@nist.gov

Combinatorial and high-throughput methods

Polymer thin films

Polymer adhesion

Mechanical properties of thin films

Surfaces and interfaces

Sun, Limin*

Isun@nist.gov

Macroporous biomaterials

Fiber-matrix interfacial shear strength

CPC composites

Taboas, Juan M.+

juan.taboas@nist.gov

Biomedical engineering

Cell and tissue mechanics

Mechanoactive bioreactors

Tissue engineering

Takagi, Shozo*

shozo.takagi@nist.gov

Crystallography

$\mathrm{X}$-ray diffraction

Calcium phosphate biomaterials

Topical fluoridation

De- and remineralization

Tesk, John A.+

john.tesk@nist.gov

Characterization: biomaterials; physical and mechanical properties

Reference biomaterials

Reference data for biomaterials

Biomaterials: orthopaedics, cardiovascular, dental, opthalmic, \& tissue engineered medical devices

Standards for medical devices

Tirumala, Vijay.+

vijay.tirumala@nist.gov

Small angle neutron scattering

Block copolymer thin films

Hydrogels

Double network hydrogels

Tominaga, Taiki.+

taiki.tominaga@nist.gov

Small angle neutron scattering

Hydrogels

Double network hydrogels

Tung, Ming S.*

ming.tung@nist.gov

Chemistry of calcium phosphate and peroxide compounds

Remineralization studies

Standard reference materials
VanderHart, David L.+ david.vanderhart@nist.gov

Measurement of orientation in polymer fibers and films

Solid-state NMR of polymers

Measurement of polymer morphology at the 2-50 nm scale

Pulsed field gradient NMR

Vogel, Brandon M.

brandon.vogel@nist.gov

Polymer synthesis

Combinatorial methods

Drug delivery

Organic electronics

Polymer thin films

Self assembled monolayers

$\mathrm{X}$-ray scattering

Solution NMR

Vogel, Gerald L.*

gerald.vogel@nist.gov

Dental plaque chemistry

Chemistry of calcium phosphates

Microanalytical techniques

Fluoride chemistry

Vogt, Bryan D.

bryan.vogt@nist.gov

Polymer thin film properties

$\mathrm{X}$-ray and neutron reflectivity

Polymers for lithography

Quartz crystal microbalance

Ordered mesoporous materials

Organic electronics

Wallace, William E.

william.wallace@nist.gov

Mass spectrometry

Geometric data analysis methods

Wang, Francis W.

francis.wang@nist.gov

Photophysics and photochemistry of polymers

Fluorescence spectroscopy

Cure monitoring of polymerization

Tissue engineering

Wang, Chengqing+

chengw@nist.gov

Small angle x-ray scattering

Critical Dimension metrology

Light scattering

Polymer and colloidal solutions 
Weir, Michael*

michael.weir@nist.gov

Biomaterials

Tissue engineering

Degradable hydrogels

Growth factor dynamics and cellular response in biomaterials

Wu, Wen-li

wen-li.wu@nist.gov

Neutron and x-ray scattering and reflectivity

Electron microscopy

Mechanical behavior of polymers and

composites

Polymer surfaces and interfaces

Polymer networks

Wu, Tao+

tao.wu@nist.gov

Combinatorial and high-throughput methods

Polymer formulations

Polymer synthesis

Interfacial tension measurements

Xu, Chang+

chang.xu@nist.gov

Combinatorial and high-throughput methods

Polymer formulations

Surface polymerization

Xu, Hockin*

hockin.xu@nist.gov

Bone tissue engineering

Scaffold and cell interactions

Fiber and whisker composites

Zhao, Hongxia (Jessica)+

jessica.zhao@nist.gov

Computed fluid dynamics

Data acquisition

Multivariate analysis

Signal processing

* Research Associate

+ Guest Scientist 


\section{National Institute of Standards and Technology}

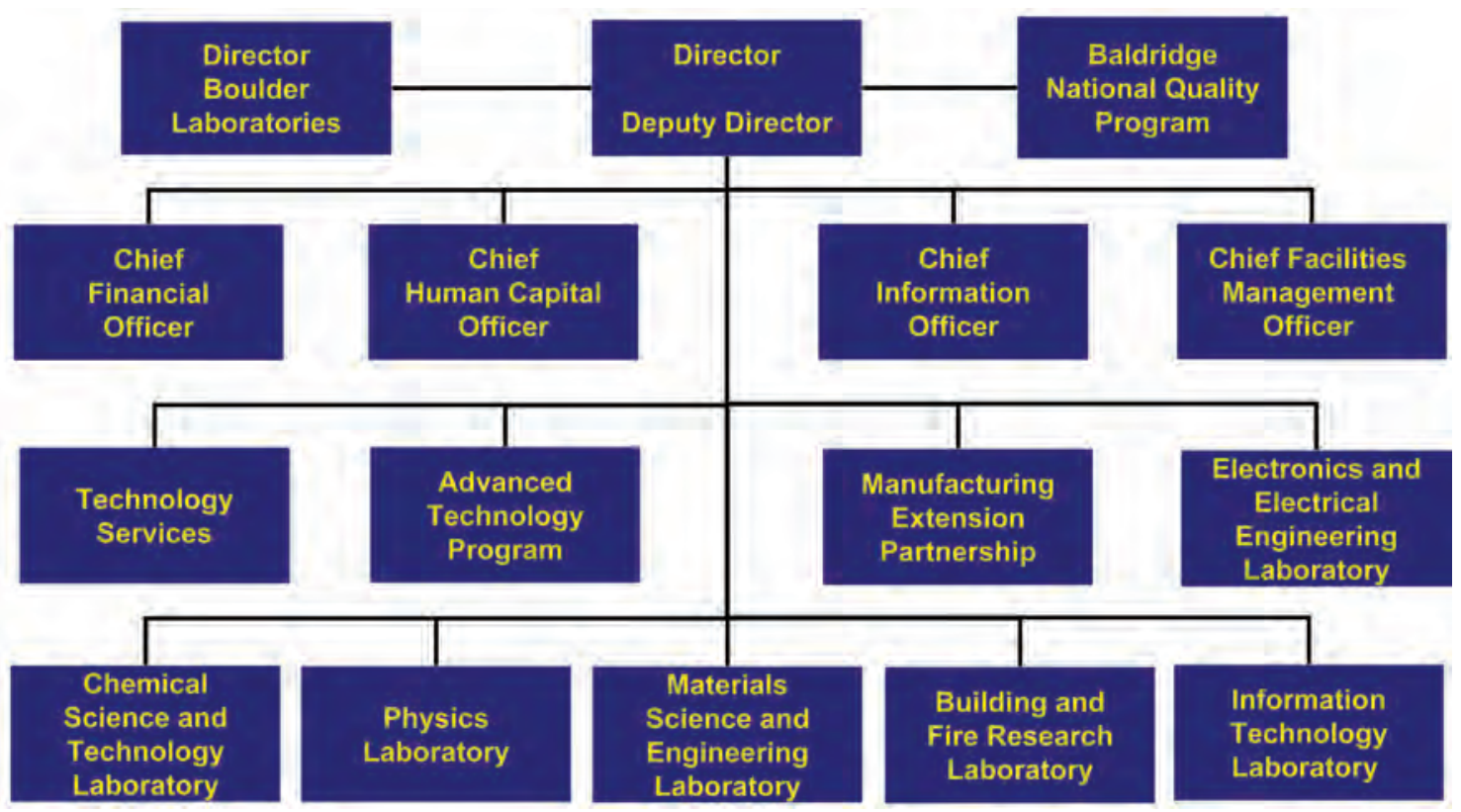

\section{Materials Science and Engineering Laboratory}

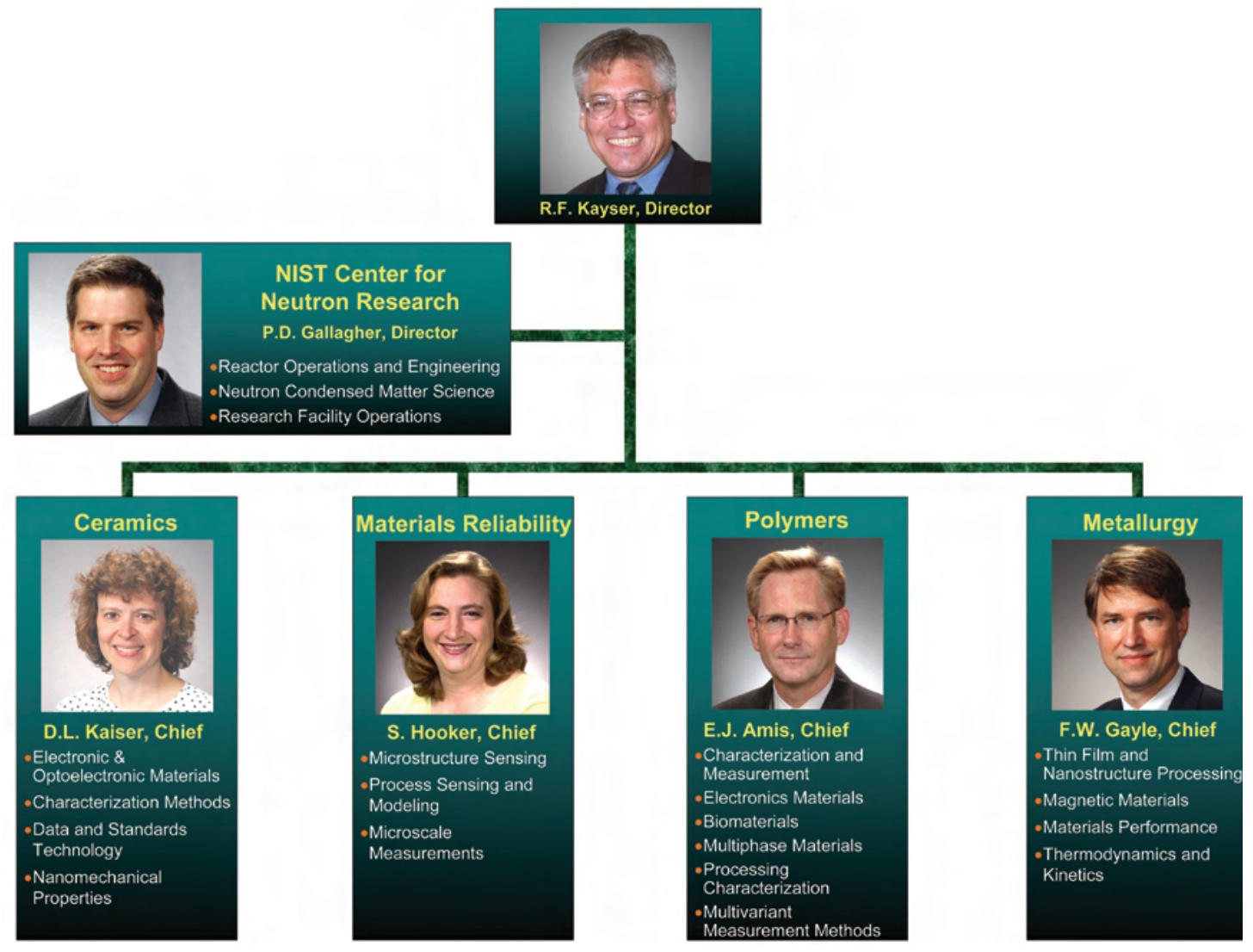




\section{Polymers Division (854.00)}

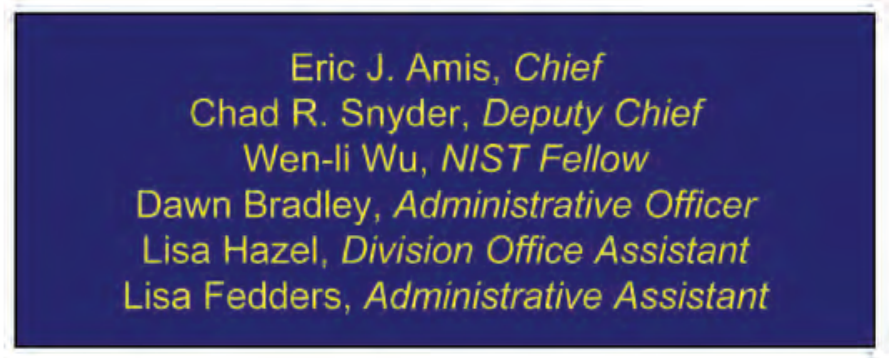

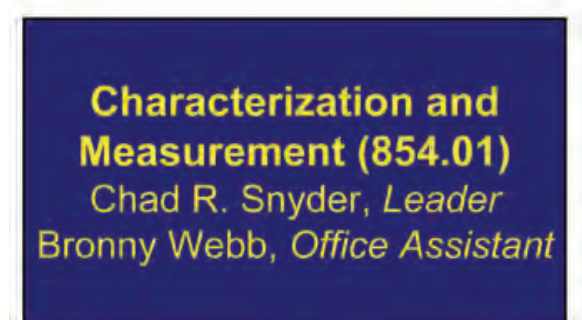

M. Chiang

K. Flynn

C. Guttman

G. Holmes

F. Khoury

D. Liu

W. McDonough

W. Wallace

\section{W. Blair}

E. DiMarzio

J. Kim

F. Landis

E. Park

J. Tesk

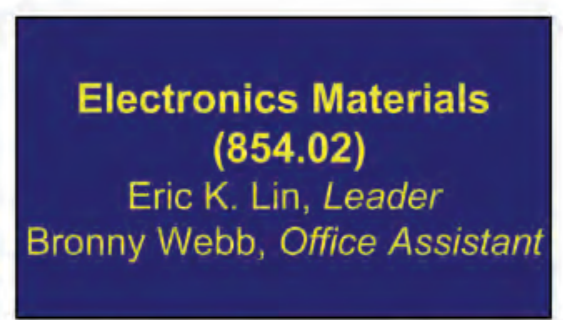

C. Chiang

D. DeLongchamp

J. Kline

K. Lavery

J. Obrzut

V. Prabhu

S. Stanley

B. Vogel
K. Choi

D. Ho

Y. Jung

S. Kang

H. Lee

T. Psurek

A. Rao

V. Tirumala

D. Vanderhart

C. Wang

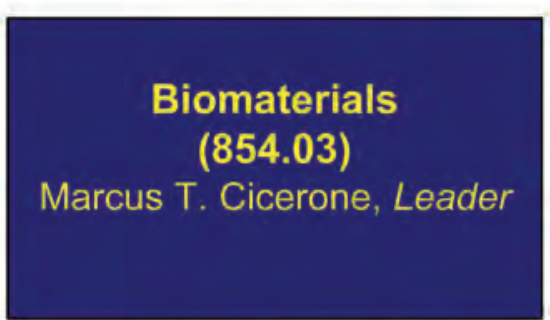

J. Antonucci

M. Becker

J. Dunkers

N. Gallant

L. Henderson

N. Lin

S. Lin-Gibson

M. Matos

L. Pakstis

M. Roy

C. Simon

F. Wang
J. Johnson

M. Kipper

Y. Lee

Y. Liu

W. Regnault

J. Sun

Y. Yang

J. Zhao
Multiphase Materials (854.04)

Alamgir Karim, Leader

Bronny Webb, Office Assistant

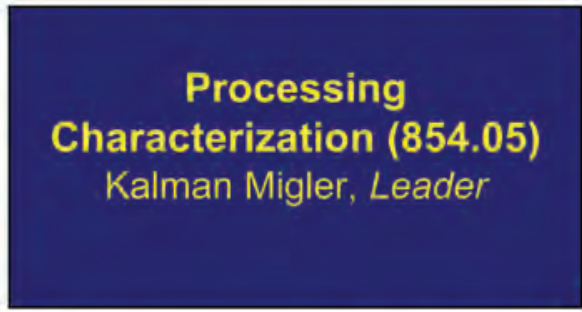

B. Bauer

J. Fagan

E. Hobbie

S. Hudson

F. Phelan
T. Chastek

H. Hu

J. Taboas

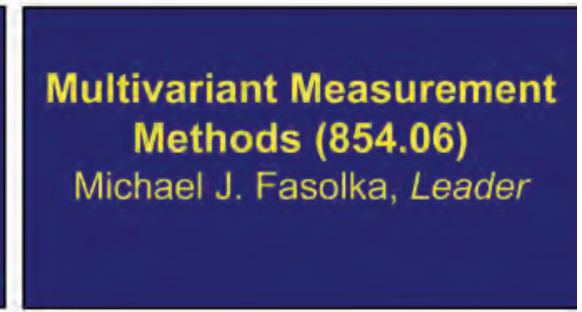
K. Beers
T. Chastek
K. Gensen
C. Laumeier
P. McGuiggan
K. Page
D. Patton
C. Stafford

A. Bur

J. Chung

$H$. Huang

K. lida

L. Lucas

H. Ró

$X$. Zhang

* Organization as of 9/29/06 
